\title{
Prodromal Alzheimer s disease in subjects with Mild Cognitive Impairment predictive and diagnostic aspects
}

Citation for published version (APA):

Ramakers, I. H. G. B. (2008). Prodromal Alzheimer s disease in subjects with Mild Cognitive Impairment predictive and diagnostic aspects. [Doctoral Thesis, Maastricht University]. NeuroPsych Publishers. https://doi.org/10.26481/dis.20080925ir

Document status and date:

Published: 01/01/2008

DOI:

10.26481/dis.20080925ir

Document Version:

Publisher's PDF, also known as Version of record

Please check the document version of this publication:

- A submitted manuscript is the version of the article upon submission and before peer-review. There can be important differences between the submitted version and the official published version of record.

People interested in the research are advised to contact the author for the final version of the publication, or visit the DOI to the publisher's website.

- The final author version and the galley proof are versions of the publication after peer review.

- The final published version features the final layout of the paper including the volume, issue and page numbers.

Link to publication

\footnotetext{
General rights rights.

- You may freely distribute the URL identifying the publication in the public portal. please follow below link for the End User Agreement:

www.umlib.nl/taverne-license

Take down policy

If you believe that this document breaches copyright please contact us at:

repository@maastrichtuniversity.nl

providing details and we will investigate your claim.
}

Copyright and moral rights for the publications made accessible in the public portal are retained by the authors and/or other copyright owners and it is a condition of accessing publications that users recognise and abide by the legal requirements associated with these

- Users may download and print one copy of any publication from the public portal for the purpose of private study or research.

- You may not further distribute the material or use it for any profit-making activity or commercial gain

If the publication is distributed under the terms of Article $25 \mathrm{fa}$ of the Dutch Copyright Act, indicated by the "Taverne" license above, 
Prodromal Alzheimer's disease in subjects with Mild Cognitive Impairment Predictive and diagnostic aspects 
(c) I.H.G.B. Ramakers, Maastricht 2008

$\begin{array}{ll}\text { Layout } & \text { Proefschrift Lay-out, Amsterdam, www.proefschriftlay-out.nl } \\ \text { Production } & \text { Datawyse BV, Maastricht } \\ \text { Publisher } & \text { NeuroPsych Publishers } \\ \text { ISBN } & 978-90-9023226-3\end{array}$

NeuroPsych Publishers is a non-profit organisation, which aims at promoting the science of 'Brain and Behaviour' and improving the application of the products of this science in health care and education. NeuroPsych Publishers accomplishes these aims by publishing books, dissertations and other products of scientific activity, by disseminating educational material and publication of tests, assessment scales and other psychometric instruments in the field of Neuropsychology, Neuropsychiatry and other areas within the domain of Brain and Behaviour.

NeuroPsych Publishers

Department of Psychiatry \& Neuropsychology

Maastricht University

P.O. Box 616

NL-6200 MD Maastricht

www.np.unimaas.nl 


\title{
Prodromal Alzheimer's disease in subjects with Mild Cognitive Impairment \\ Predictive and diagnostic aspects
}

\author{
Proefschrift
}

Ter verkrijging van de graad van doctor aan de Universiteit Maastricht, op gezag van de Rector Magnificus, Prof. mr. G.P.M.F. Mols, volgens het besluit van het College van Decanen, in het openbaar te verdedigen op donderdag 25 september 2008 om 14:00 uur.

door

Inez Hubertina Gertruda Bernard Ramakers

Geboren op 19 november 1979 te Kerkrade 
Promotores

Prof. dr. F.R.J. Verhey

Prof. dr. J. Jolles

Co-promotor D. P.J. Visser

Beoordelingscommissie Prof. dr. M. Limburg (voorzitter)

Dr. P. Aalten

Prof. dr. H. Crebolder

Dr. A.F.G. Leentjens

Prof. dr. B. Schmand

The research described in this thesis was performed at the Department of Psychiatry \& Neuropsychology, School of Mental Health and Neuroscience, Maastricht University, The Netherlands.

Financial support for the publication of this thesis was kindly provided by Alzheimer Nederland, Internationale Stichting Alzheimer Onderzoek (ISAO), Servier, Lundbeck B. V., Novartis Pharma B.V., Wyeth, Janssen-Cilag, Nutricia Advanced Medical Nutrition, and IPC Neurobrain B.V. 


\section{CONTENT}

$\begin{array}{lll}0 & \text { Prologue } & 7\end{array}$

$1 \quad$ Introduction 13

$\begin{array}{ll}\text { Part } 1 & 21\end{array}$

$2 \quad$ The predictive value of memory strategies for Alzheimer's disease in 23 subject with Mild Cognitive Impairment.

Submitted

3 The association between APOE genotype and memory dysfunction in subjects with Mild Cognitive Impairment is related to age and Alzheimer pathology.

Accepted for publication in Dementia and other Geriatric Cognitive Disorders

$4 \quad$ Affective symptoms as predictor of Alzheimer's disease in subjects with Mild Cognitive Impairment: a 10-year follow-up study.

Submitted

5 Symptoms of preclinical dementia in general practice up to five years before dementia diagnosis.

Published in Dementia and other Geriatric Cognitive Disorders, 24, 300-306.

Part 2

6 Determinants of help-seeking behaviour in subjects with subjective memory complaints at a memory clinic: a case-control study. Accepted for publication in the International Journal of Geriatric Psychiatry

7 Memory clinics in the Netherlands: also a service of non-demented subjects with cognitive complaints?

Published in Tijdschrift voor Gerontology en Geriatrie, 38, 237 - 245.

$8 \quad$ Concluding remarks 
Summary

Samenvatting

References

123

Dankwoord

Curriculum vitae

139

Publications and abstracts 
Prologue 
This thesis deals with the diagnostic evaluation of subjects with $\mathrm{MCl}$. We would like to start with two case histories to exemplify the common diagnostic issues with these subjects.

\section{Case A}

A 66-year old man, Mr. A, was referred by his GP to the memory clinic for the evaluation of his increasing forgetfulness and concentration problems. Mr. A presented with slowly progressive forgetfulness and problems with concentration over approximately the past two years. He forgot names, could not remember details of his last holiday and forgot what someone had told him. He feared to become demented, like his mother. Mr. A asked the clinician some questions: Are the symptoms suggestive of early dementia or could there be another cause for the complaints? Would the symptoms increase in the future?

Mr. A was a friendly man who appeared a little bit nervous. He retired three years ago, but remained active through his hobbies, doing volunteer work, and taking care of his grandchildren. Somatic, neurologic, psychiatric, and laboratory assessments were unremarkable. General cognitive functioning was normal (MMSE $=28)$, with a very mild decline in functioning (Global Deterioration Scale, GDS $=2$ ), very mild impairments in daily functioning (Blessed Dementia Rating Scale, BDS $=0.5$ ), and very mild symptoms of depression (Hamilton Depression Rating Scale, HDRS = 1). Additional diagnostics were applied. The MRI-scan showed some atrophy of the medial temporal lobe (score is 1). The neuropsychological assessment showed a high level of intelligence, corresponding with his high level of education. Memory scores were below average (-1.3 SD), but did not fulfil the criteria of a formal impairment. Mr. A showed an inefficient use of memory strategies in the learning phase. In addition, he had increased distractibility in the inhibition of an automatic respons. Other cognitive domains, including speed, visuoconstruction, language, and working memory performances were within normal ranges. Based on the neuropsychiatric, neuropsychological, and laboratory assessments and on the MRI-findings, the conclusion was that Mr. A was not demented, but because of some decreased test performances in memory and executive functioning, and the very mild medial temporal lobe atrophy, a decrease in functioning compared to his previous level could not be excluded. For monitoring his cognitive functioning, Mr. A participated in the Maastricht follow-up study, which has follow-up assessments after 2, 5 and 10 years. As part of this study the APOE genotype was determined, and showed that Mr. A carried one APOE e4 allele. 


\section{2-years follow-up}

Mr. A reported an increase of memory and concentration problems. He forgot names and appointments. He often did not know what he was doing and had problems with doing two things simultaneously. His wife confirmed these problems. He tried to cope with the complaints by using an agenda and making lists of things to do. His interest in things, which are more effortful at the moment, decreased. He sometimes felt a bit sad. There were no changes in hobbies or housekeeping activities. General cognitive functioning was mildly impaired (MMSE $=25$, GDS $=3$ ). Daily functioning was not impaired as demonstrated with a BDS of 0 . There was evidence of mild depressive symptoms (HDRS $=8$ ). The neuropsychological assessment showed low scores on concept shifting, delayed recall of a word learning test and the immediate recall and delayed recall of a story, but not severe enough to fulfil the criteria of an impaired test score. Compared to the baseline assessment there was a decline in memory functioning, cognitive speed, and IQ. The diagnostic team decided that the symptoms were not of sufficient severity for a diagnosis of dementia, but were too prominent for normal aging. The course of the symptoms raised the suspicion that the symptoms could be early signs of preclinical dementia.

\section{5-years follow-up}

Mr. A reported increasing forgetfulness. He often forgot to use his agenda, had to read things again, forgot the contents of books he had read last week and had increasing problems with finding the way in unfamiliar places. General cognitive functioning remained stable, and depressive symptoms were only very mild. Test performances showed impairment in the delayed recall of unstructured information and impairments in concept shifting. Functioning in daily life was mildly impaired as demonstrated with a BDS of 1.5. His spouse reported mild changes in behaviour such as irritability, and taking less initiative. The diagnosis made was cognitive disorder not otherwise specified.

\section{0-years follow-up}

After nine years, Mr. A's wife contacted the memory clinic for a new appointment, because she noted severe progression of the problems in cognitive functioning and daily functioning of her husband. Mr. A had severe forgetfulness, such as problems with remembering conversations, news from the television or newspaper, and names, also of family members. In addition, he said things over and over again. In daily functioning, he experienced problems with driving, problems with his orientation, and problems with the financial administration. He was less interested in his hobbies or in visiting friends. At home, he only did things when he was stimulated by his spouse. He had no problems with mood or sleeping, but he had lost some 
weight in the past year. General cognitive functioning was impaired (MMSE $=22$ ), and there was significant impairment in daily functioning $(B D S=4)$. On the neuropsychological assessment there were multiple cognitive deficits in the domains of memory, speed, executive functioning, verbal fluency and visuoconstruction. The diagnosis of Alzheimer type dementia was made. The wife of Mr. A asked whether there were other tests to confirm this diagnosis. The clinician adviced to repeat the MRI scan and to measure beta amyloid and tau protein in cerebrospinal fluid.

\section{Case B}

Mrs. B was referred to the memory clinic by her GP, because she was worried about her memory problems. She reported that she had to write down everything, and forgot appointments she had made the day before. Mrs. B was a 66-year old, friendly and communicative woman, with a moderate level of education. She stopped working one year previously and noted memory complaints since that time point. Her husband did not report any changes in daily functions, he only reported signs of irritability and nervousness. Mrs B worried a lot about her forgetfulness, also at night when she could not sleep. She wanted to know whether she was becoming demented or not. And, if so how long this process would take.

General cognitive and daily functions were very mildly impaired. There were mild depressive symptoms (HDRS score of 11). There were no abnormalities on neurologic, somatic or laboratory examinations. The MRI-scan showed no abnormalities. The neuropsychological assessment showed below average memory performances on the immediate recall and delayed recall of a word list, but a normal performance on the recognition part of the test. Other performances were within normal limits. The neuropsychologist remarked that Mrs. $B$ was very nervous, mainly about the memory tests. Mrs. B also participated in the followup study. Her performances after 2, 5 and 10 years varied over time. Memory performances improved to normal levels at the two and five years follow-up, whereas executive functioning declined at the five-years follow-up, but remained stable between the 5 and 10-years followup. At the 10-years follow-up cognition was within normal limits and it was concluded that her memory problems were probably related to depressive symptomatology and normal aging.

Both cases fulfilled the criteria of $\mathrm{MCl}$ at baseline, but showed a different course with a different outcome over the follow-up period of 10 years. Some of the questions that arise from these case reports are: 
- Which factors at baseline could have predicted the difference in course and outcome of the cognitive problems in case $A$ and $B$ ?

- Could the GP have foreseen this course, and was the referral to the memory clinic correct?

These questions that are common in clinical practice formed the basis for the research described in this thesis. 

Introduction

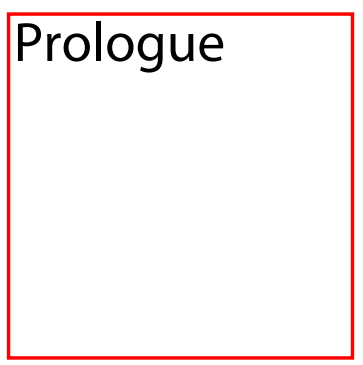




\subsection{Cognitive impairments in non-demented subjects}

Problems in cognitive functioning, as reported by Mr A and Mrs B in Chapter 0, are very common in middle-aged and elderly subjects. The most frequently reported problem is forgetfulness. The prevalence of forgetfulness in non-demented subjects varies from $41 \%$ in subjects between 55 and 65 years old to $52 \%$ in subjects over 70 years (Ponds et al., 1997). These cognitive problems can be part of the normal aging process, but they can also be very early symptoms of dementia. The phase between normal cognitive functioning and dementia is often called Mild Cognitive Impairment (MCl). In this phase, subjects experience cognitive problems and a decline in functioning compared to a previous level of functioning that is greater than would be expected based on age and educational level. Still, the cognitive and functional impairments of subjects with $\mathrm{MCl}$ are not severe enough to meet the criteria for dementia. Subjects with $\mathrm{MCI}$ mainly experience problems with memory functioning, but may also have impairments in other cognitive domains such as attention, executive functioning or language (Dickerson et al., 2007). The prevalence of $\mathrm{MCl}$ in older adults ranges from approximately 11 to $17 \%$ in community studies depending on cohort source and definition (Ganguli, 2006; Mariani et al., 2007).

In the last 20 years, there has been a lot of interest in subjects with $\mathrm{MCl}$, as these subjects are at increased risk for developing dementia, especially Alzheimer's disease (AD). Longitudinal and prospective studies have reported conversion rates from $\mathrm{MCl}$ to AD from 6$25 \%$ per year, compared to a conversion rate to AD of $1-2 \%$ in the general population (Feldman \& Jacova, 2005; Geslani et al., 2005; Panza et al., 2008; Petersen et al., 1999; Visser et al., 2006). However, not all subjects with $\mathrm{MCl}$ will develop $\mathrm{AD}$, and the cognitive impairment can remain stable or even improve over time (Visser et al., 2002). Cognitive impairment that remains stable or improves over time is unlikely to be caused by AD and may result from several psychological, somatic or social aspects (Jorm et al., 2004; Ponds et al., 1997; Zimprich et al., 2003). This shows that the natural course of $\mathrm{MCl}$ is heterogeneous and uncertain. Thus, generally subjects with $A D$ have experienced a phase of $M C l$ prior to the diagnosis of $A D$, but not all subjects with $M C I$ have prodromal AD (Figure 1.1).

\section{Mild Cognitive Impairment}
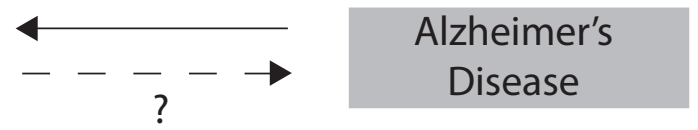

\section{Figure 1.1}




\subsection{Research challenges in $\mathrm{MCl}$}

Because of the aging population, the prevalence of $\mathrm{MCl}$ will increase in future. Also the number of subjects with dementia in The Netherlands is estimated to increase from about 200.000 in 2000 to more than 400.000 subjects in 2050 (Health Counsil of the Netherlands, 2002). This implies that the impact of $\mathrm{MCl}$ and $\mathrm{AD}$ on the health care system will increase rapidly in the following years. With no current pharmacological therapy available, which can intervene with the degenerative process and stop the disease progression, it is still important to investigate subjects with $\mathrm{MCl}$ for several scientific and clinical reasons. From a scientific point of view it is important to identify subjects with $\mathrm{MCl}$ who will develop AD. The characteristics of these preclinical subjects and the course of their complaints will give more insight into the underlying mechanisms of pathological aging and can be useful for the identification of factors that predict $A D$. When pharmacological treatment, with drugs with disease modifying properties, becomes available this will be most effective in the earliest stages of the disease. Therefore, the identification of subjects at risk is very important for screening purposes.

From a clinical point of view, it is important to differentiate between probable progressive and non-progressive $\mathrm{MCl}$, as in both groups the cause of the cognitive complaints will be different and both groups of subjects will benefit from other sorts of advice and treatment. In addition, a reliable prognosis may give subjects the opportunity to anticipate on their future.

\subsection{Role of health care settings in the evaluation of subjects with cognitive impairments}

In the Dutch health care system, the general practitioner (GP) is normally the first person who is contacted by subjects with cognitive complaints or their relatives. GP's often know their patient for a longer time and are therefore in an excellent position to detect gradual changes in cognitive and behavioural functioning. For these reasons the GP could have an important role in the identification of subjects at risk for dementia (Artero \& Ritchie, 2003; Barrett et al., 1996; Wilkinson et al., 2004) and subjects who could benefit from additional diagnostics and referrals to specialized facilities such as memory clinics. However, the symptoms that are suspected for future cognitive decline or dementia in a general practice setting remain largely unknown.

Secondary health care facilities for the evaluation of cognitive complaints are outpatient clinics for neurological, psychiatric and geriatric patients. As cognitive impairments can have multiple causes, it has been recognized that the evaluation of cognitive impairments should follow a multidisciplinary approach. For this reason specialized services, often referred 
to as Memory Clinics (MCs), have been set up. Memory clinics are relatively new facilities in the health care system. In The Netherlands, the first MCs developed in 1986 in Maastricht and Tilburg (Verhey et al., 1987). These settings have a multidisciplinary way of working and are involved in the early diagnosis and treatment of people with dementia (Lindesay et al., 2002; Wright \& Lindesay, 1995). An overview about the organization and working methods of memory clinics in The Netherlands was published in 1998 (Verhey et al., 1999). The findings of the 12 MCs that were operational at that time showed that MCs were mainly associated with university hospitals, and were mainly involved in the diagnostic process of dementia. However, the role of memory clinics in subjects with milder cognitive problems, such as subjects with $\mathrm{MCl}$ or no cognitive disorder, is largely unclear. In addition, it is unclear which people seek help at a memory clinic for their cognitive problems and which people do not.

\subsection{Predictors for outcome in $\mathrm{MCI}$}

The heterogeneity in cause and prognosis of cognitive complaints increases the need for sensitive predictors, which can determine whether subjects with cognitive impairments will progress to $A D$ or not. In the last 20 years, several predictors have been identified which increase the predictive accuracy of $\mathrm{MCl}$ for AD. These predictors include increased age (Reiman \& Caselli, 1999; Visser et al., 2002), female gender (Gao et al., 1998), low level of education (Reiman \& Caselli, 1999; Schmand et al., 1997), cognitive measures (Dickerson et al., 2007; Saxton et al., 2004; Twamley et al., 2006), medial temporal lobe atrophy (DeCarli et al., 2007; Twamley et al., 2006), and cerebrospinal fluid levels of beta-amyloid and tau (Mariani et al., 2007). However, none of these predictors can predict AD with sufficiently high accuracy. In this thesis we will focus on a number of predictors.

\subsubsection{Memory impairment}

One of the strongest predictors of $A D$ is impairment in memory functioning, mainly in episodic memory (Sarazin et al., 2007; Saxton et al., 2004; Twamley et al., 2006; Visser et al., 2002) which is often measured with total scores on immediate or delayed recall tests. However, not all subjects with memory impairment develop AD. Memory performances can be enhanced by the use of strategies during the learning of new information (Saan \& Deelman, 1986; Sternberg \& Tulving, 1977). It is known that the effective use of these strategies is impaired in subjects with AD (Delis et al., 1991). However, the predictive value of these strategies for the development of $A D$ in subjects with $\mathrm{MCl}$ is not clear and should be established further, as this could give more insight into the underlying mechanisms of memory performances and memory impairments in subjects with $\mathrm{MCl}$ and may help in the identification of subjects with prodromal AD. 


\subsubsection{APOE genotype}

The apolipoprotein E (APOE) e4 allele is a major genetic risk factor for Alzheimer's disease (AD) (Petersen et al., 1995), and is a predictor for AD in subjects with $\mathrm{MCl}$. In addition, it is associated with memory impairment both in subjects with $A D$ and non-demented subjects (Small et al., 2004; van der Vlies et al., 2007). The relation between the APOE e4 genotype and memory dysfunctioning in subjects with $\mathrm{MCl}$ is less clear. In addition, it is unclear whether the effect of the APOE genotype on memory functioning is caused by underlying AD pathology or is an independent effect. The effect of the APOE e4 allele on several aspects of memory is important, because it could increase the understanding of the underlying pathology of $\mathrm{MCl}$.

\subsubsection{Non-cognitive symptoms}

Besides impairments in cognitive functioning, behavioural problems are also common in subjects with AD and MCI (Aalten et al., 2005; Apostolova \& Cummings, 2007; Lopez et al., 2005; Lyketsos et al., 2002). Most commonly reported are symptoms of depression, anxiety, apathy and irritability. Some studies found that the presence of these symptoms was associated with an increased risk for the development of dementia (Modrego \& Ferrandez, 2004; Palmer et al., 2007; Robert et al., 2006). However, there is no consensus in the literature about the predictive value of these affective symptoms for $A D$ as other studies found a negative or no association between affective symptoms and AD (Copeland et al., 2003; Feldman et al., 2007; Korf et al., 2004; Liu et al., 2007; Palmer et al., 2007; Panza et al., 2008; Robert et al., 2006; Rozzini et al., 2005; Teng et al., 2007; Tierney et al., 1999; Visser et al., 2000; Visser et al., 2000; Wang et al., 2006). Conflicting results may be explained by differences in selection of the subjects (setting and used $\mathrm{MCl}$-criteria), measurement of the affective symptoms, age at baseline or length of follow-up. However, the conflicting findings from previous studies are remarkable and should be established further.

\subsection{Aim of the thesis}

The main aim of this thesis was to investigate the course of cognitive complaints in subjects with $\mathrm{MCl}$ and to identify early predictors of dementia, especially AD. In this thesis we wanted to investigate the following objectives:

(a) Which learning strategies are predictive for the development of $A D$ in subjects with $\mathrm{MCI}$ ?

(b) What is the effect of the APOE genotype on memory function in middle-aged and older subjects with $\mathrm{MCl}$ ? 
(c) Which non-cognitive symptoms are predictive for $\mathrm{AD}$ in subjects with $\mathrm{MCl}$ ?

(d) Which symptoms can predict dementia in a general practice setting?

(e) What are the determinants of subjects who seek help for their subjective memory complaints at a memory clinic?

(f) How did memory clinics in The Netherlands develop, and which role do they play for subjects with $\mathrm{MCl}$ ?

\subsection{Outline of this thesis}

This thesis is organized as follows. The chapters can be divided into two parts. In part I, we focused on the predictive value of several risk factors for $A D$ in subjects with $\mathrm{MCl}$. There are several definitions present in the literature for describing the phase between normal cognitive functioning and dementia. In the present thesis, $\mathrm{MCl}$ is used to refer to subjects, who had subjective memory complaints in the absence of dementia, and had a Global Deterioration Scale (Reisberg et al., 1982) score of 2 or 3 , and no significant impairment in general cognitive functioning or daily functioning. Also other studies have used these criteria (Feldman \& Jacova, 2005; Tabert et al., 2006; Tierney, Szalai, Snow, Fisher, Tsuda et al., 1996).

In Chapter 2, we investigated the predictive accuracy of an active learning strategy (subjective organization), and a more passive rehearsal based strategy (serial clustering) for AD. We performed a prospective longitudinal study with follow-up assessments after 2, 5 and 10 years among subjects with $\mathrm{MCl}$ form the Maastricht Memory Clinic.

In Chapter 3, we investigated the relation between the APOE e4 genotype and several aspects of verbal memory in subjects with $\mathrm{MCl}$ from the Maastricht Memory Clinic. In addition, we investigated whether the effect of the APOE e4 allele on memory functioning is different in middle-aged and old subjects.

In Chapter 4, the predictive accuracy of several affective symptoms for the development of $A D$ was investigated using a prospective longitudinal study with a follow-up period up to 10 years. We investigated the predictive accuracy of depression, anxiety, apathy, sleeping problems and somatic symptoms. We also investigated whether the effect of affective symptoms on $\mathrm{AD}$ was dependent on age, the presence of amnestic $\mathrm{MCl}$, and length of followup up to 10 years.

In Chapter 5, we investigated the predictive value of cognitive impairment, affective symptoms, behavioural problems, vascular problems, gait disturbances, and changes in appetite and weight for future dementia in general practice settings. For this study, we used data from the Registration Network of Family Practices (RNH). We performed a case-control design, matched for age, sex and general practice setting. 
In part II, we focused on the memory clinic settings. Subjects with cognitive problems or their family often feel uncomfortable with their complaints and often worry about their impairments. These subjects are often referred to Memory Clinics for the evaluation of their complaints.

In Chapter 6, we investigated which factors are determinants of seeking help at a memory clinic for subjective memory complaints in subjects without objectified memory impairment. In this study, we compared cases from the Maastricht Memory Clinic to control subjects from the Maastricht aging study who did not seek help for their subjective memory complaints. We investigated the following symptoms: depression, anxiety, memory selfefficacy, quality of life, changes in memory and daily functioning, and worries caused by a family history of dementia.

The development of memory clinics in The Netherlands in the past 10 years is described in Chapter 7. We sent an inventory to all MCs that were operational in The Netherlands at that time. In addition, this chapter investigated the role of this facility for non-demented subjects such as subjects with $\mathrm{MCl}$ or subjects with subjective cognitive complaints.

Lastly, in Chapter 8, we summarized the main findings of this thesis, and we discussed the usefulness of the present study for further research and clinical practice. 



$$
\text { Part } 1
$$





\section{The predictive value of memory}

strategies for Alzheimer's disease in subjects with Mild Cognitive Impairment

Ramakers, IHGB., Visser, PJ., Aalten, P., Maes, HL., Lansdaal, HGM., Meijs, CJC., Jolles, J. \& Verhey, FRJ. 


\section{Abstract}

Background. Subjects with Alzheimer's disease (AD) show impaired learning strategies. Whether impaired learning strategies are already present in subjects with prodromal AD remains unknown. The aim of the present study was to investigate the predictive accuracy of learning strategies for $A D$ in subjects with Mild Cognitive Impairment (MCI).

Methods. Subjects with $\mathrm{MCl}(\mathrm{n}=284)$ were selected from the Maastricht Memory Clinic. Subjects were and reassessed over a period of 10 years. Sixty of the 284 subjects convert to AD. Learning strategies investigated were subjective organization and serial clustering.

Results. Lower scores of subjective organization were associated with a higher risk for $A D$ at follow-up $(\mathrm{OR}=1.74,95 \% \mathrm{Cl}=1.16-2.66, \mathrm{p}=0.008)$. Serial clustering did not predict $A D$. Findings were similar in subjects aged $40-59$ years and subjects aged $60-85$ years.

Conclusion. Prodromal AD is characterized by impaired learning strategies. This finding may have implications for the early detection of $A D$ in subjects with $\mathrm{MCl}$ and for the development of cognitive training programs.

Key words: Mild cognitive impairment, Alzheimer's disease, learning strategies, predictors 


\section{Introduction}

Mild Cognitive Impairment $(\mathrm{MCl})$ is common among the elderly. It is a relevant health care problem because it reduces the quality of daily living, and because a subgroup of subjects with $\mathrm{MCl}$ show a progressive course and will develop Alzheimer's disease (AD) in the future (Visser et al., 2006). It remains difficult to identify AD among subjects with $\mathrm{MCl}$. This would be important because drugs that have the potential to modify the disease progression are supposed to be most effective in the early stage of the disease.

The main feature of $A D$ are impairments in encoding and retrieval of new information. Encoding is facilitated by the use of learning strategies (Saan \& Deelman, 1986; Sternberg \& Tulving, 1977), and there is evidence that subjects with AD do not use learning strategies efficiently (Delis et al., 1991). Previous studies reported that, when compared to healthy controls, subjects with AD showed significantly lower average levels of semantic clustering (Carlesimo et al., 1998; Perri et al., 2005), and did not benefit from the semantic relatedness of the presented stimuli (Carlesimo et al., 1998). Also subjects with $\mathrm{MCl}$ less often used semantic clustering as a learning strategy compared to control subjects (Perri et al., 2005; Ribeiro et al., 2007). Therefore, impaired learning strategies could be a predictor for $A D$ in subjects with $M C l$, but this has not yet been investigated.

The main aim of this study is to investigate the predictive accuracy of learning strategies for $A D$ in subjects with $\mathrm{MCl}$. Learning strategies that will be investigated are subjective organization and serial clustering. Subjective organization refers to constancies in the order of responses, that develop over a series of free recalls. Subjective organization requires an active reorganization of the presented words (Bousfield \& Bousfield, 1966; Saan \& Deelman, 1986; Sternberg \& Tulving, 1977). In contrast, serial clustering is an automatic, more passive strategy in which the words are recalled in the same order as they were offered (Delis et al., 1987). Because subjective organization requires more effort and is less automatic than serial clustering, we hypothesised that subjective organization would be impaired in early $A D$ and would be predictive for AD at follow-up, while serial clustering would be not.

\section{Methods}

\section{Subjects}

Subjects were selected from an ongoing, prospective, longitudinal study of non-demented subjects referred to the Maastricht Memory Clinic. The memory clinic is an outpatient clinic at the Maastricht University Hospital in the Netherlands (Verhey et al., 1993). Subjects were referred to the memory clinic for evaluation of their cognitive complaints. The design of this 
study has been described in detail elsewhere (Verhey et al., 1993; Visser et al., 2006; Visser \& Verhey, 2007; Visser et al., 2000). Consecutive patients were included at the time of the first visit to the memory clinic. Inclusion criteria were age 40 years or older and having $\mathrm{MCl}$, defined as a score of 2 or 3 on the Global Deterioration Scale (GDS) (Reisberg et al., 1982). This broad definition of $\mathrm{MCl}$ is consistent with that of other studies (Feldman \& Jacova, 2005; Tabert et al., 2006; Tierney et al., 1996). It was used because subjects with AD in the predementia stage do often not meet more strict criteria of $\mathrm{MCl}$, such as criteria for amnestic $\mathrm{MCl}$ (Geslani et al., 2005; Storandt et al., 2006; Visser \& Verhey, 2008). Exclusion criteria were dementia at baseline and an apparent cause for their cognitive impairment, such as cerebrovascular disorders, brain trauma, endocrine disorders or psychiatric disorders other than mild affective disorders at baseline (Visser et al., 2000). For the present study, we selected subjects with the same version of the 15-item Auditory Verbal Learning Task (AVLT, see below) (Brand \& Jolles, 1985; Van der Elst et al., 2005) and who had been eligible for the first follow-up visit (see below). The baseline characteristics of the study sample $(n=284)$ are shown in Table 1 . The study was approved by the medical ethical Committee at Maastricht University Hospital. After the study was explained to the subjects, all subjects gave their written informed consent.

\section{Clinical assessment and diagnosis at baseline}

At baseline, all subjects underwent a standardised assessment, which included a detailed history of the subject, a psychiatric, neurological, and physical examination, the Mini Mental State Examination (MMSE) (Folstein et al., 1975), assessment using clinical rating scales (i.e. GDS (Reisberg et al., 1982), Hamilton Depression Rating Scale (HDRS) (Hamilton, 1960) and the Blessed Dementia Rating Scale (BDRS) (Blessed et al., 1968)), appropriate laboratory tests (i.e. tests for hematology, glucose, biochemical analyses, vitamin B12, and thyroid stimulating hormone), a neuropsychological assessment including tests covering the domains of memory, attention, executive functioning, language, praxis, and intelligence (Verhey et al., 1993), and CT or MRI as described elsewhere (Verhey et al., 1993). The diagnosis of dementia and AD was made according to the DSM-IV and NINCDS-ADRDA criteria (APA, 1994; McKhann et al., 1984).

\section{Clinical assessment and diagnosis at follow-up}

Two, five, and ten years after baseline, subjects were invited to participate in a follow-up assessment. Because enrolment was continuous, not all subjects had completed all followup assessments. The number of subjects eligible for the 2-year follow-up was 348, for the 5year follow-up 382, and for the 10-year follow-up 217. The follow-up assessment included a standardized questionnaire about medical history and cognitive complaints, the MMSE, GDS, BDS, HDRS, and an extensive neuropsychological assessment comparable to baseline. Subjects 
who refused to come for the follow-up assessment were assessed via a telephone interview, which included a standardized questionnaire about medical history and cognitive complaints, and the Telephone Interview for Cognitive Status (Brandt et al., 1988). The diagnosis of dementia was based on the DSM IV criteria (APA, 1994) and was made independently by both a neuropsychiatrist and a neuropsychologist. Both professionals were blinded to the baseline results. If disagreement on the diagnosis occured, a consensus meeting was held. Subjects who were diagnosed as being demented at the first follow-up (two years after baseline), were not invited for the next follow-up (five years after baseline). The outcome after two, five, and ten years was defined as the subject had developed AD or had no dementia.

\section{Memory strategies}

Memory strategies were tested using the Dutch version of the AVLT at baseline (Van der Elst et al., 2005). Fifteen unrelated monosyllabic words were presented five times and after each presentation the subject was asked to recall as many words as possible. After 20 minutes, during which non-verbal tests were performed, delayed recall was tested. The strategies of subjective organization and serial clustering were investigated using the five learning trials of the AVLT.

\section{Subjective organization}

Subjective organization refers to the consistencies in response order, that develop over a series of free recalls (Bousfield \& Bousfield, 1966; Tulving, 1962). Based on the output order in which the words from two successive trials are listed by the subject, combinations (clusters) of words that are recalled together in these two successive trials are identified. These combinations are based on the organization schema developed by the individual subject, and are not dependent on prespecified categorization based on semantic relationships, which is used in several word learning tests such as the California Verbal Learning Test (Delis et al., 1987).

A bidirectional inter-trial repetition (ITR) measure was used (Sternberg \& Tulving, 1977). This measure is a variant of the unidirectional ITR measure, first proposed by Bousfield \& Bousfield (1966). In the bidirectional ITR, comparisons were made between the forward and backward sequence of recalled words in two successive trials. Each time a combination of two words is recalled consecutively in one trial and also in the following trial (= Inter-Trial Repetition) the subjects gets one point. The bidirectional ITR corrects for clusters that occur by chance with the following formula (Sternberg \& Tulving, 1977): Subjective organization = Observed (ITR) - Expected (ITR), where expected ITR $=2 c(c-1) / h * k$. Observed ITR is the number of pairs of items recalled on trial $t$ and $t+1$ in adjacend output positions in either of the two possible orders. Expected ITR is the expected number of pairs of items, $c$ was the number 
of common items recalled in both trials $t$ and $t+1, h$ was the number of items recalled in trial $t$, and $k$ was the number of items recalled in trial $t+1$. An outcome of 0 indicated that the number of observed ITRs is equal to the number of expected ITRs. A score higher than 0 indicates more use of subjective organization than expected by chance.

\section{Serial clustering}

Serial clustering refers to the number of word pairs that are reproduced in the same order as they were presented (Delis et al., 1988; Kramer et al., 1997). This form of clustering is a more passive approach to recall. To measure serial clustering, we used an adapted form of the formula devised by Mulder et al. (1996). In this formula, the total number of observed serial clusters is divided by the number of clusters that are expected based to occur on change (Bouma et al., 1996; Mulder et al., 1996; Saan \& Deelman, 1986). The expected number that would be generated by chance is calculated by dividing the number of all possible clusters by the total number of words recalled in that trial. A positive score indicates that the subjects reported more clusters than would be expected generated by chance.

The difference between subjective organization and serial clustering is that in serial clustering the subject reproduce the items in the same order as they were presented, whereas in subjective organization the subjects has to reorganize the presented items into clusters that are logical to the subjects by keeping the other items in mind.

\section{Statistical analyses}

Statistical analyses were performed using the Statistical Package for Social Sciences (SPSS) for Macintosh, version 11. To analyse differences between converters and non-converters, independent t-tests were conducted for continuous variables, and chi-square tests for level of education and dichotomous variables. Level of education was classified as low (at most primary education), middle (junior vocational education), or high (senior vocational education or academic training) (UNESCO, 1976). The predictive accuracy was analyzed using a discretetime survival analysis model and implemented using logistic regression and an appropriately adjusted dataset (Hosmer \& Lemeshow, 1999). The outcome group included all subjects with $A D$ at follow-up. The reference group included subjects without dementia at follow-up. Subjects with a non-AD type dementia at follow-up were excluded from the analyses. Analyses were done with and without adjustment for age, sex, and education. The average level of subjective organization was defined as the sum of the four trial-pairs divided by four. The average level of serial clustering was defined as the sum of each of the trial-scores divided by five. Because the risk for AD is dependent on age (Visser et al., 2006; Visser et al., 2000), we also repeated analyses in two subgroups (40-59 years and 60-85 years). Two-factor repeated measures ANOVAs were 
performed, with group as a between factor, trial as a within factor, and age, education, and sex as covariates, to analyze the course of learning strategies across the 5 learning trials of the AVLT at baseline. In order to investigate the relation between the use of learning strategies and delayed recall performance, we performed correlation analyses. All tests were two-tailed, and the significance level was set at 0.05 .

\section{Results}

The diagnosis at the latest follow-up for which the subject was eligible was available for 284 of the 348 subjects (82\%). The outcome was unavailable for 64 of the subjects because: (a) the subjects did not want to participate in the follow up $(n=15)$, (b) the subject was untraceable $(n=24)$, (c) the subject had passed away $(n=17)$, (d) the subjects suffered severe illness $(n=1)$, or other reasons $(n=7)$. Subjects with completed follow-up were about 3 years younger and had lower GDS and BDS scores than subjects who did not complete the follow-up. Both groups were comparable with respect to education, sex distribution and scores on the MMSE, HDRS, and z-score on the delayed recall test of the AVLT. Of these 284 subjects 60 (21\%) subjects had developed $A D$ within the follow-up period up to 10 years, 31 after 2 years, 19 after 5 years, and 10 after 10 years. Table 1 shows the baseline characteristics of the subjects who developed AD

Table 1. Characteristics of subjects who developed AD and who did not.

\begin{tabular}{|c|c|c|c|}
\hline & $\begin{array}{l}\text { Not demented } \\
(n=224)\end{array}$ & $\begin{array}{l}A D \\
(n=60)\end{array}$ & p-value \\
\hline Age, $M \pm S D$ & $55.6 \pm 9.3$ & $67.8 \pm 8.0$ & $<0.001$ \\
\hline Sex, $\%$ man & 59 & 48 & 0.141 \\
\hline Education, \% low/moderate/high & $17 / 47 / 36$ & $27 / 45 / 28$ & 0.200 \\
\hline$M M S E, M \pm S D$ & $28.6 \pm 1.5$ & $26.6 \pm 2.2$ & $<0.001$ \\
\hline $\mathrm{HDRS}, \mathrm{M} \pm \mathrm{SD}$ & $10.0 \pm 6.5$ & $8.0 \pm 5.6$ & 0.032 \\
\hline $\mathrm{BDS}, \mathrm{M} \pm \mathrm{SD}$ & $1.1 \pm 1.3$ & $1.9 \pm 1.7$ & 0.003 \\
\hline $\mathrm{GDS}, \mathrm{M} \pm \mathrm{SD}$ & $2.3 \pm 0.5$ & $2.6 \pm 0.5$ & $<0.001$ \\
\hline Delayed recall, $\mathrm{z}$-score, $\mathrm{M} \pm \mathrm{SD}$ & $-0.50 \pm 1.2$ & $-2.3 \pm 1.0$ & $<0.001$ \\
\hline Learning, $z$-score, $\mathrm{M} \pm \mathrm{SD}$ & $-0.58 \pm 1.2$ & $-1.9 \pm 1.0$ & $<0.001$ \\
\hline Subjective organization* ${ }^{*} \mathrm{M} \pm \mathrm{SD}$ & $1.2 \pm 1.0$ & $0.7 \pm 0.8$ & $<0.001$ \\
\hline Serial clustering* ${ }^{*} \mathrm{M} \pm \mathrm{SD}$ & $3.0 \pm 1.5$ & $2.7 \pm 1.7$ & 0.138 \\
\hline
\end{tabular}

$\mathrm{M}=$ mean, $\mathrm{SD}=$ standard deviation, $\mathrm{MMSE}=$ Mini Mental State Examination, $\mathrm{HDRS}=$ Hamilton Depression Rating Scale, GDS = Global Deterioration Scale, BDS = Blessed Dementia Rating Scale * = Mean over the learning trials 
and those who did not. Subjects with AD at follow-up had a significantly lower baseline MMSE score, a higher GDS and BDS score, a lower HDRS score, a lower z-score on the delayed recall test of the AVLT, and were older than those without AD at follow-up. Sex distribution, and level of education were comparable in both groups.

The average score of the strategies used in the five learning trials of the two groups are presented in Table 1. Converters had significantly lower mean levels of subjective organization. Serial clustering was comparable in both groups.

Two factor GLM repeated measures ANOVA analyses, with age, sex and educational level as covariates showed a significant main effect of group on subjective organization ( $F$ $=5.7 ; \mathrm{df}=1 ; \mathrm{p}=.017$ ), indicating that converters had significant lower scores of subjective organization across the learning trials than non-converters (Figure 1a). The main effect for trial and the interaction effect between group and trial did not reach statistical significance. For serial clustering, the main effects for group and trial, and the interaction effect between group and trial were not statistically significant (Figure 1b).

Survival analyses corrected for baseline age, education, and sex showed that lower levels of subjective organization were associated with an increased risk for $A D(O R=1.7,95 \%$ $C l=1.16-2.66, p=0.008)$. Serial clustering was not predictive for $A D$ at follow-up $(p=0.438)$.

In the age subgroups similar findings were obtained. Decreased use of subjective organization was associated with an increased risk for AD in both age subgroups (subjects 40-

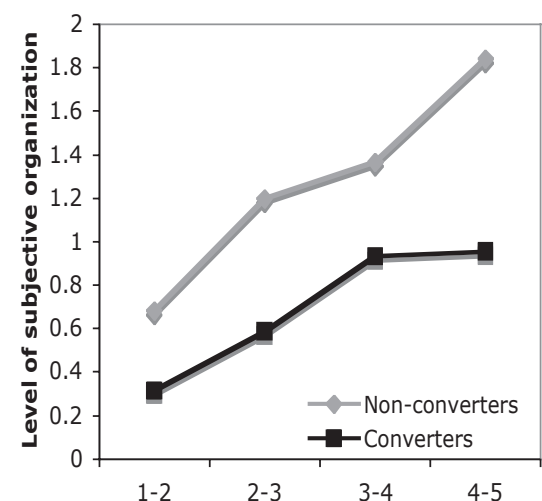

A.

Trial

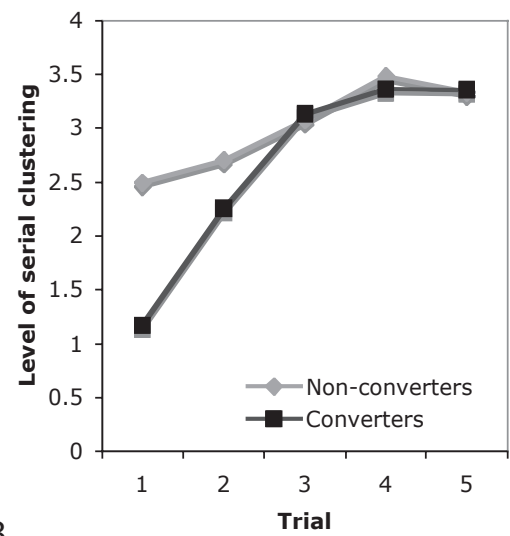

B.

Figure 1: Average levels of learning strategies in converters and non-converters to $A D . A$ : Subjective organization, B: Serial clustering. The black line represents the converters, the grey line the non-converters. 
59 years old: $\mathrm{OR}=3.1,95 \% \mathrm{Cl}=0.97-9.9, \mathrm{p}=0.055$; subjects $60-85$ years old: $\mathrm{OR}=1.6,95 \% \mathrm{Cl}$ $=1.01-2.5, p=0.046)$. Serial clustering was not predictive for $A D$ in either age group.

In order to investigate whether subjective organization was associated with progression to $A D$ at the short term or long term, we performed separate analyses for subjects who were diagnosed with $A D$ after 2, 5, and 10 years. The $O R$ for AD after 2 years ( 31 subjects with $A D$ ) was 1.96 , for $A D$ after 5 years ( 19 subjects with $A D) 1.03$, and for $A D$ after 10 years (10 subjects with AD) 4.5 .

In order to investigate the relation between the use of learning strategies and delayed recall performance, we performed correlation analyses. There was a significant relation between subjective organization and delayed recall performance $(r=0.49, p<0.001)$, but not with serial clustering.

\section{Discussion}

Less use of subjective organization was associated with an increased risk for AD. Use of serial clustering was not predictive for AD.

Serial clustering is a passive, low-level approach to recall, which does not involve any new transformation or organization of the presented information (Glosser et al., 2002). Subjective organization, on the other hand, requires more effort and an active reorganization of the presented information by keeping the other items accessible in the mind. This effortful strategy consumes a greater portion of a person's limited mental resources (Bjorklund \& Douglas, 1997), and seems to be problematic for subjects with $\mathrm{MCl}$ who later convert to AD. Our findings correspond with the findings of a cross-sectional study of Carlesimo et al. (1998), which suggested that memory aspects that require more processing resources can be use to differentiate normal from pathological aging. This is consistent with the idea that more passive on rehearsal based strategies develop earlier in childhood and deteriorate later in life, while more complex organization strategies develop later in childhood (Schneider \& Sodian, 1997) and degenerate earlier in the elderly. Lower scores of subjective organization were associated with an increased risk for $A D$ both at the short term and at the long term, indicating that impairments in subjective organization is a very early symptom of AD. Why subjective organization was not predictive for AD after 5 years is not clear and may be a chance finding.

Subjective organization depends on active reorganization of the incoming information, and may therefore be related to prefrontal lobe functioning and executive functioning (Fletcher et al., 1998; Fletcher et al., 1998; Kramer et al., 2005; Savage et al., 2001). In order to investigate whether the effect of subjective organization on AD was mediated by an impairment in executive function, we post-hoc repeated the analyses with 
correction for Stroop card III score, TMT B score, and fluency (Reitan, 1958; Stroop, 1935; Van der Elst et al., 2006a, 2006b). In these analyses, subjective organization remained a significant predictor for $A D$, which suggests that subjective organization problems can not be explained by impairments in executive functioning, as measured with the tests we used in the present study.

We found that subjective organization was associated with the delayed recall performance at baseline. This suggests that subjective organization improves learning and subsequent retrieval from long-term memory.

\section{Implications}

The finding that subjective organization was already impaired in prodromal AD gives new insights in the cognitive mechanisms that underlie memory dysfunction in subjects with prodromal AD. In clinical practice, subjective organization scores may help to predict AD in subjects with $\mathrm{MCl}$. The observed correlation between subjective organization and delayed recall performance supports the use of cognitive training in subjects with $\mathrm{MCl}$ (Belleville, 2008) and may help the development of new cognitive training programmes which take learning strategies into account. Because of the role of learning strategies in prodromal $A D$, the type of word list used to assess memory function may be important for the ability to detect of subjects with AD. Word lists that include semantic related words might be less useful to identify subjects with prodromal $A D$, because active reorganization of the presented material is less needed. Word list learning tests in which the order of the words are presented varies over the learning trials, such as the word learning test of the Consortium to Establish a Registry for Alzheimer's disease (Welsh et al., 1994) or the cognitive part of the Alzheimer's Disease Assessment Scale (Rosen et al., 1984), might make it more difficult for subjects to apply subjective organization or serial clustering strategies and might be sensitive for early AD.

To our knowledge, this study was the first longitudinal study investigating the use of memory strategies in subjects with $\mathrm{MCl}$ and their predictive accuracy for $\mathrm{AD}$ in a large clinical sample with a follow-up up to 10 years. The post-hoc calculation indicated that based on the means of subjective organization the power of the total study was .97 at a significance level of .05. Nonetheless, this study does have some points of attention. One issue worth mentioning is that the possibility to use strategies is related to the number of recalled words after each learning trial. However, we corrected the cluster scores for the total number words remembered in each learning trial. Additionally, the course of memory strategies over time was not investigated in this study. We did exclude subjects with a non-AD type dementia at follow-up. However, when we repeated the analyses with including non-AD type dementia in 
the no AD group, results were comparable. The results of this study can be applied to subjects from a memory clinic, but may not be generalisable to other settings.

In summary, we found that the ineffective use of effortful strategies was associated with an increased risk for AD. This finding may help to identify subjects with prodromal AD in subjects with $\mathrm{MCl}$ and may help to develop cognitive training programs. 



\section{The association between APOE genotype and memory dysfunction in subjects with}

Mild Cognitive Impairment is related to age and Alzheimer pathology

Ramakers, IHGB., Visser, PJ., Aalten, P., Bekers, O., Sleegers, K., van Broeckhoven, KL., Jolles, J. \& Verhey, FRJ. 


\section{Abstract}

Background: Memory problems are a main feature of Mild Cognitive Impairment ( $\mathrm{MCl}$ ) and may be related to the Apolipoprotein E (APOE) e4 allele. We investigated whether the effect of the APOE genotype on memory in subjects with $\mathrm{MCl}$ was dependent on age and underlying Alzheimer disease (AD) pathology.

Methods: Subjects with $\mathrm{MCl}$ older than 40 years $(n=181)$ were selected from a memory clinic setting. Subjects with at least one APOE e4 allele $(n=84)$ were compared to non-carriers on learning, delayed recall, intrusions, retention rate, subjective organization, serial clustering, primacy and recency effect with a multi-trial verbal learning test. Subjects were reassessed 5 to 10 years later in order to identify those who developed AD. Analyses were performed in the total sample and in a middle-aged (age 40-59) and elderly subgroup (age 60-85).

Results: In the total sample, the APOE e4 allele was most strongly related to impaired learning. In the middle-aged subgroup, the APOE e4 allele was most strongly related to decreased subjective organization and in the old subgroup to a decreased delayed recall. After excluding subjects with incipient $A D(n=34)$, results remained similar in the middle-aged subgroup, but in the old subgroup the APOE genotype was no longer associated with memory dysfunction.

Conclusion: The presence of the APOE e4 allele is associated with impaired memory functioning in both middle-aged and old subjects with $\mathrm{MCl}$, although the memory function affected varies with age. Its effect on memory function may be dependent on underlying AD pathology in elderly subjects, but not in middle-aged subjects.

Key words: APOE, MCl, Alzheimer's disease, memory strategies. 


\section{Introduction}

The apolipoprotein E (APOE) e4 allele is a major risk factor for Alzheimer's disease (AD) (Petersen et al., 1995) and is associated with memory impairment both in subjects with AD and nondemented subjects (Small et al., 2004; van der Vlies et al., 2007; Wilson et al., 2002). The APOE e4 allele may also affect on memory function in subjects with mild cognitive impairment (MCl) (Albert et al., 2007; Farlow et al., 2004; Smith et al., 1998). MCI refers to cognitive impairment in subjects without dementia, and is associated with an increased risk for AD (Visser et al., 2006). The relation between the APOE e4 allele and memory function in $\mathrm{MCl}$ is still poorly understood. It is not clear whether the effect of APOE on memory function is mediated by underlying AD pathology (Bennett et al., 2005), or is an independent effect of the APOE genotype (Martins et al., 2006). In addition, in order to better understand the relation between APOE genotype and memory function it would be important to know whether the effect of APOE genotype is dependent on age, because underlying pathology in MCl may vary with age (Nilsson et al., 2006; Small et al., 2004). In addition, it would be important to know which aspect of memory is most severely affected, because this may given an indication of which brain regions are involved. Previous studies in subjects with $\mathrm{MCl}$ however, were conducted in elderly subjects only (Albert et al., 2007; Farlow et al., 2004), and mainly investigated learning and delayed recall.

The aims of the present study were to investigate the effect of the APOE e4 allele on memory function in middle-aged (40 - 59 years) and elderly (60 - 85 years) subjects with $\mathrm{MCl}$ from a memory clinic setting and to investigate whether the effect of APOE e4 allele on memory function was dependent on underlying AD pathology. To that end we followed subjects for 5 to 10 years and repeated analyses after exclusion of subjects who developed AD during this period. Besides learning and delayed recall, we also investigated memory measures that have not been investigated before such as primacy, recency, retention, intrusions, subjective organization, and serial clustering. Subjective organization and serial clustering are learning strategies that are used by a subject to learn new information. These strategies are impaired in early AD (Carlesimo et al., 1998; Delis et al., 1991; Perri et al., 2005).

\section{Methods}

\section{Subjects}

Subjects were selected from an ongoing longitudinal study of non-demented subjects referred to the Maastricht memory clinic. The memory clinic is an outpatient clinic of the university hospital Maastricht (Verhey et al., 1993). The design of the study has been described in detail elsewhere (Verhey et al., 1993; Visser et al., 2006). Consecutive patients were included at the time 
of the first visit to the memory clinic. Inclusion criteria were age 40 years or older, and had $\mathrm{MCl}$, defined as a score of 2 or 3 on the Global Deterioration Scale (GDS) (Reisberg et al., 1982). This broad definition of $\mathrm{MCl}$ is consistent with that of other studies (Feldman \& Jacova, 2005; Tabert et al., 2006; Tierney et al., 1996). It was used because subjects with AD in the predementia stage do often not meet more strict criteria of $\mathrm{MCl}$, such as criteria for amnestic $\mathrm{MCl}$ (Geslani et al., 2005; Storandt et al., 2006; Visser \& Verhey, 2007). Exclusion criteria were dementia at baseline and an apparent cause for their cognitive impairment, such as cerebrovascular disorders, brain trauma, endocrine disorders or psychiatric disorders other than mild affective disorders at baseline (Visser et al., 2000). Subjects included in this study were referred to the memory clinic by general practitioners (76\%), neurologists (6\%), psychiatrists $(12 \%)$ or others $(6 \%)$.

For the present study we selected those subjects eligible for the five or ten year follow-up ( $n=454)$. During this period five versions of the Auditory Verbal Learning Test (AVLT) (Brand \& Jolles, 1985) were used at baseline. In order to avoid a version effect of the AVLT, we only included subjects with the version that was most commonly used $(n=368)$. Subjects with this version had similar age, sex, level of education, MMSE score, GDS score and BDS score, as subjects with other versions of the AVLT. Next we excluded subjects on whom APOE genotyping had not been performed $(n=187)$. The final study sample consisted of 181 subjects. Data on APOE genotype was mainly unavailable because blood for genotyping was collected at follow-up during a specific time period. In addition, a few subjects refused APOE genotyping. Subjects whose APOE status was not available were significantly older (60.7 vs. 57.0 years, $\mathrm{p}=.001$ ) compared to subjects whose APOE status was known. Both groups were comparable with respect to educational level, sex distribution, MMSE score, HDRS score and total delayed recall at baseline, as well as percentage of subjects with $A D$ at follow-up.

\section{Clinical assessment at baseline and follow-up}

At baseline, all subjects underwent a standardised assessment, which included a detailed history of the subject, a psychiatric, neurological, and physical examination, the Mini Mental State Examination (MMSE) (Folstein et al., 1975), GDS (Reisberg et al., 1982), Hamilton Depression Rating Scale (HDRS) (Hamilton, 1960) and the Blessed Dementia Rating Scale (BDS) (Blessed et al., 1968), appropriate laboratory tests, a neuropsychological assessment, and a CT or MRI as described elsewhere (Verhey et al., 1993). For describing purposes we subdivided subjects at baseline in $4 \mathrm{MCl}$ groups based on the neuropsychological test performances in the domains of memory, attention, executive functioning, language, and visuoconstruction, as described elsewhere (Visser et al., 2006). Performance in a cognitive domain was considered impaired in the score on at least one test from that domain was below -1.5 standard deviation (SD) of age, sex and education corrected means of healthy control subjects (Van der Elst et al., 2005; Van 
der Elst et al., 2006b) except for the measure of visuoconstruction in which impairment was based on a qualitative rating (Visser et al., 2006). Subjects without impairment in any domain were classified as subjective complaints $(n=53)$; subjects with only impairments in one or more non-memory domain as non-amnestic $\mathrm{MCl}(\mathrm{naMCl})(n=66)$, subjects with impairment in only the memory domain as amnestic $\mathrm{MCl}$ single domain (aMCI-SD) $(n=13)$, and subjects with both impairments in memory and non-memory domains as amnestic $\mathrm{MCl}$ multiple domain (aMCl-MD) ( $n=48)$. One subject could not be classified, because non-memory tests had not be done. The criteria for naMCl and $\mathrm{aMCl}$ also require intact activities of daily living (Petersen, 2004). This was operationalized as the absence of dementia and a score below 4 on the GDS, which was part of the inclusion criteria of the study, in agreement with previous studies (Geslani et al., 2005).

Two, five and ten years after baseline, the subjects were invited for a follow-up assessment. This assessment included a standardized questionnaire about medical history and cognitive complaints, the MMSE, GDS, BDS, HDRS, and an extensive neuropsychological assessment comparable to the baseline assessment. Subjects who refused to come for the follow-up were assessed via a telephone interview, which included a standardized questionnaire about medical history and cognitive complaints, and the Telephone Interview for Cognitive Status (Brandt et al., 1988). The diagnosis of dementia and AD was made independently by both a neuropsychiatrist and a neuropsychologist according to the DSM-IV and NINCDSADRDA criteria (APA, 1994; McKhann et al., 1984). Both were blinded to the baseline results. If disagreement on the diagnosis occurred, a consensus meeting was held. Subjects, who were diagnosed as being demented at follow-up, were not invited for the next follow-up.

\section{Outcome at follow-up}

The average follow-up duration was 8.7 years. Because blood for genotyping was collected at follow-up, all subjects had at least one follow-up. Of the 181 subjects included at baseline, 140 subjects had been in the study long enough to be contacted for the 10-year follow-up and cognitive outcome was available for $120(86 \%)$ of them, the other subjects $(n=41)$ only had a five year follow-up. Reason for no outcome at the 10-year follow-up was because the subjects refused $(n=6)$, had died $(n=7)$, or were untraceable $(n=7)$. Subjects with and without cognitive outcome had comparable age, sex distribution, level of education and MMSE score. During the total follow-up period 34 subjects (19\%) had developed AD. After 5 years 26 subjects had developed $A D$, and after 10 years 8 . Of those with $A D$ at follow-up, 6 were younger than 60 years and 28 were older than 60 years. 


\section{Measures of memory impairment}

In the Dutch version of the AVLT (Brand \& Jolles, 1985; Van der Elst et al., 2005) fifteen unrelated monosyllabic words are presented five times in the same order and after each presentation the subject is asked to recall as many words as possible. After 20 minutes, in which non-verbal tests were performed, delayed recall was tested. The AVLT can be used to investigate the following aspects of verbal memory functioning:

Learning: total of correctly remembered words from the 5 immediate recall trials.

Delayed recall: total number of correctly remembered words after 20 minutes.

Retention: total delayed recall score divided through the highest score at the 5 immediate recall trials, multiplied by 100 .

Intrusion: number of recalled words in the 5 immediate recall trials that were not part of the AVLT.

Subjective organization: Subjective organization refers to the constancies in response ordering, that develop in a series of free recalls (Bousfield \& Bousfield, 1966; Tulving, 1962). Based on the output order in which the words from two successive trials are listed by the subjects, combinations (clusters) of words that are recalled together in these two successive trials are identified. These combinations are based on the organization schema developed by the individual subject, and are not dependent on prespecified categorization based on semantic relationships, which is used in several word learning tests such as the California Verbal Learning Test (Delis et al., 1987). A bidirectional inter-trial repetition (ITR) measure was used (Saan \& Deelman, 1986; Sternberg \& Tulving, 1977). Each time a combination of words is recalled consecutively in one trial and also in the next trial (= inter-trial repetition) the subject gets one point. The bidirectional ITR corrects for clusters that occur by chance with the following formula: Subjective organization = observed (ITR) - expected (ITR), where expected ITR $=2 c(c-1) / h{ }^{*} k$ (Saan \& Deelman, 1986; Sternberg \& Tulving, 1977). Observed ITR is the number of pairs of items recalled on trial $t$ and $t+1$ in adjacent output positions in either of the two possible orders. Expected ITR is the expected number of pairs of items, $c$ is the number of common items recalled on both trials $t$ and $t+1, h$ is the number of items recalled on trial $t$, and $k$ is the number of items recalled on trial $t+1$. An outcome of 0 indicates that the number of observed ITRs is equal to the number of expected ITRs. A score higher than 0 indicates more use of subjective organization than expected by chance.

Serial clustering: Serial clustering is a measure for the reproduction of words in the same order as that they were presented. This form of clustering indicates a more passive approach to recall. Serial clustering was calculated with correction for the number of clusters that can be expected to occur by chance (Mulder et al., 1996; Saan \& Deelman, 1986). 
Primacy effect: ratio of the number of words remembered from the first 3 words of the list divided by 3.

Recency effect: ratio of the number of words remembered from the last 3 words of the list divided by 3 .

\section{Apolipoprotein E genotyping}

APOE genotype was determined on genomic DNA extracted form EDTA anticoagulated blood using the polymerase chain reaction (PCR) technique (Bekers et al., 2002; Slooter et al., 1998; Wenham et al., 1991). Genotyping was done blinded for all clinical data. Eighty-four subjects (46\%) had one or two e4 alleles (APOE+), viz. e4e4 ( $n=9,5 \%)$, e3e4 ( $n=69,38 \%)$, and e2e4 $(n=6,3 \%)$. Ninety-seven subjects (54\%) had no e4 allele (APOE-), viz. e3e3 $(n=76,42 \%)$, e2e3 $(n=20,11 \%)$, and e2e2 ( $n=1,0.5 \%)$.

\section{Statistics}

Statistical analyses were performed using the Statistical Package for Social Sciences (SPSS) for the Macintosh, version 11. To analyse baseline differences between carriers and non-carriers of the APOE e4 allele independent $t$-tests were used for continues variables, and Chi-square tests for categorical variables. Level of education was classified into low (at most primary education), middle (junior vocational education), or high (senior vocational education or academic training) (UNESCO, 1976). Univariate variance analyses (ANOVA) adjusted for age, education and sex were used to test group differences in memory performance between carriers and non-carriers of the APOE e4 allele. Stepwise binairy logistic regression analysis with correction for age, sex and education was used to identify the memory variable to which the APOE e4 allele was strongest related. All analyses were repeated without subjects who had developed $A D$ within the study period of 10 years and were performed in the total group and in subjects aged 40 to 59 years (middle-aged subgroup) and 60 to 85 years (older subgroup). To adjust for multiple comparisons we used an a-level of 0.01 .

\section{Results}

\section{Baseline characteristics}

Baseline characteristics of carriers and non-carriers of the APOE e4 allele are presented in Table 1. In the total group, APOE e4 carriers were about 4 years older, had a significantly lower MMSE and delayed recall score, a slightly higher GDS score, a lower HDRS score, and more often $\mathrm{aMCl}(46$ vs $24 \%, \mathrm{p}=0.003$ ) compared to non-carriers. Educational level and sex distribution were comparable. In the middle-aged subgroup, baseline characteristics and distribution of 
$\mathrm{MCl}$-subtypes was comparable between subjects with and without the APOE e4 allele. In the elderly subgroup, APOE e4 carriers had a lower score on the delayed recall test and were more often aMCl-MD (42 vs. 19\%, $\mathrm{p}=0.045$ ) than subjects without the APOE e4 allele. The APOE e4 allele was more common in subjects older than 60 years (58\%) than in the middle-aged subgroup (40\%) $(p=.015)$.

\section{Effect of APOE on memory function}

Memory performances of carriers and non-carriers of the APOE e4 allele are presented in Table 2. In the total sample, carriers of the e4 allele had lower scores on learning, delayed recall, subjective organization and primacy. In subjects younger than 60 years, APOE e4 carriers had lower scores on learning and subjective organisation, while in the elderly subgroup APOE e4 carriers had lower scores on learning and delayed recall (Table 2).

Binary logistic regression analyses, adjusted for age, sex and education showed that in the total sample the APOE e4 allele was most strongly related to the learning performance $(p=.003)$. In middle-aged subjects, the APOE e4 allele was most strongly related to subjective organization ( $p=.011)$, while in the elderly subjects the APOE e4 allele was most strongly related to the delayed recall performance $(p=.02)$.

\section{Effect of underlying AD pathology}

When the subjects with AD at follow-up were excluded from the analyses (6 in the 60- group, and 28 subjects in the $60+$ group), the APOE e4 allele was associated with lower scores on learning in the total group $(p=0.025)$. In middle-aged subjects, the APOE e4 allele was still associated with lower scores on subjective organization $(p=0.028)$, but no longer with learning. In the $60+$ group, the APOE genotype was no longer associated with any of the memory measures.

\section{Discussion}

In the present study, we found an effect of the APOE e4 allele on various aspects of memory in subjects with $\mathrm{MCl}$, which depends on age and underlying AD pathology. In elderly subjects, the APOE e4 allele was most strongly related to delayed recall performance, compared to subjective organization in the middle-aged subgroup. The effect of APOE on memory function may have been related to underlying $A D$ pathology in subjects over 60 years, but not in middleaged subjects.

The effect of the APOE e4 genotype on delayed recall, and learning in elderly subjects with $\mathrm{MCl}$ is in line with previous studies (Albert et al., 2007; Smith et al., 1998). It suggests involvement of the medial temporal lobe as this region plays an important role in these 


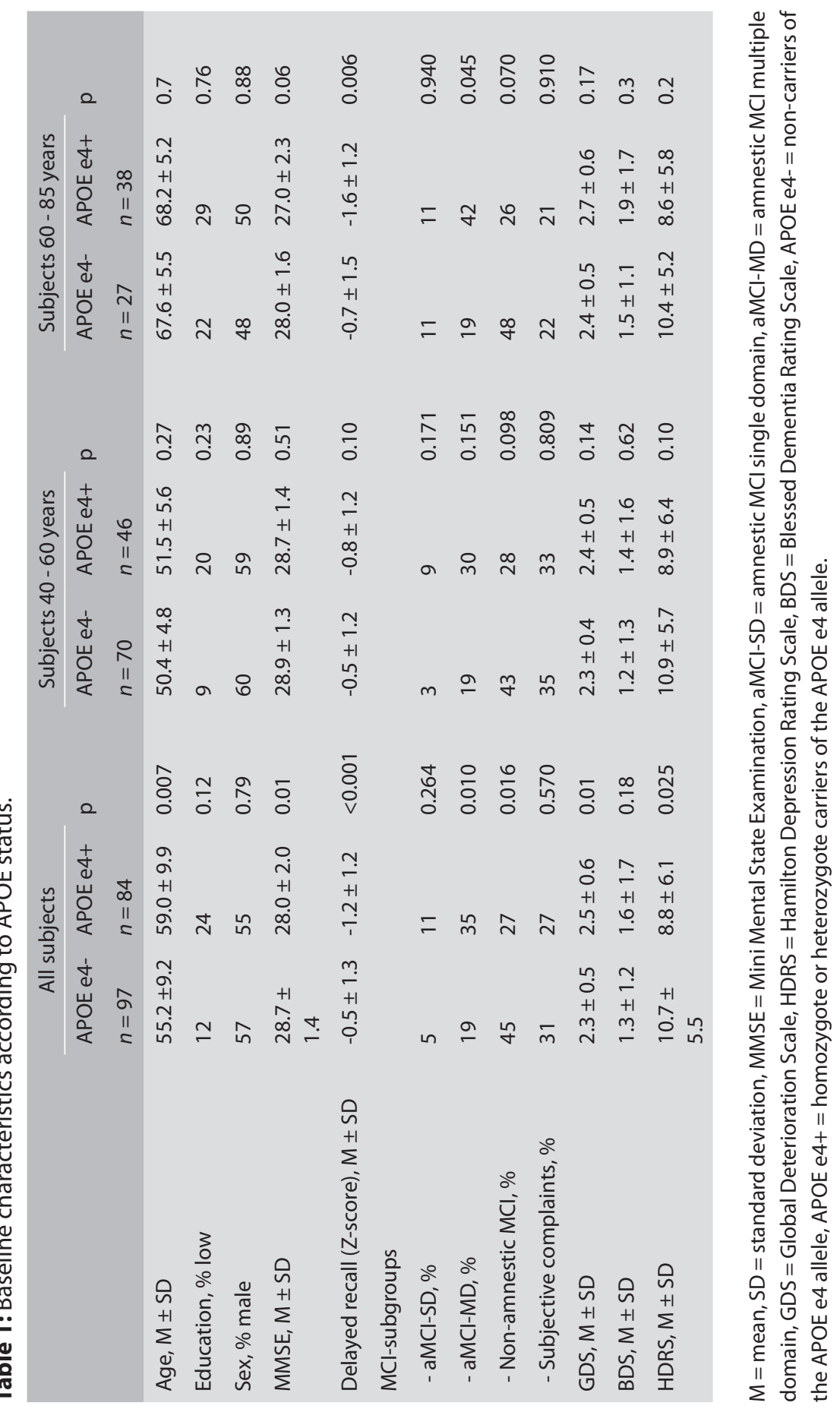




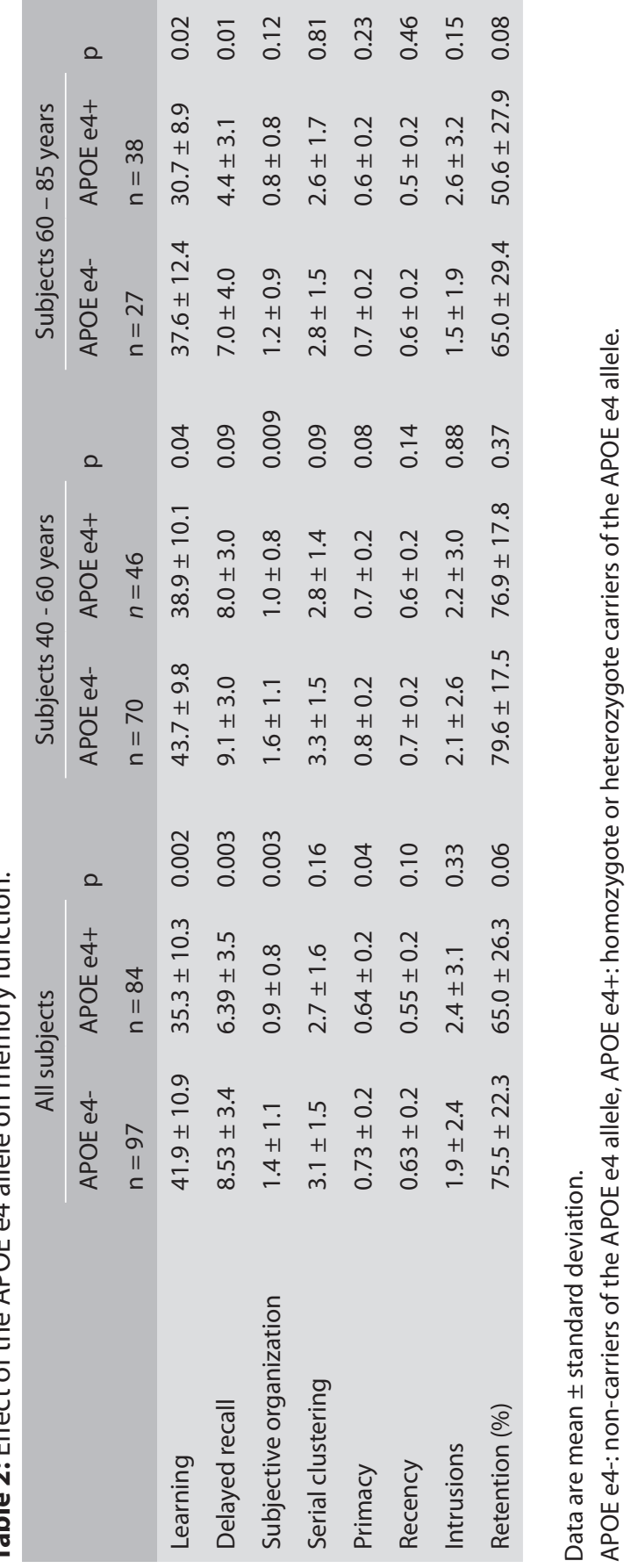


memory functions. Indeed, there is evidence that elderly subjects with $\mathrm{MCl}$ with an APOE allele have more atrophy in these region compared to subjects without an APOE allele (Fleisher et al., 2005). The effect of the APOE e4 allele on memory functioning may have been modulated by underlying $A D$ pathology, since after exclusion of subjects who developed $A D$, the APOE genotype was no longer associated with memory performance. This finding is consistent with a population-based study in elderly subjects which showed that the APOE is no longer associated with cognition after correction for amyloid load in the brain (Bennett et al., 2005). However, the lack of an effect of APOE genotype on memory after exclusion of incipient AD cases might also have resulted from a lack of statistical power, because after the exclusion of AD subjects only 10 subjects older than 60 years carried the APOE e4 allele.

In subjects younger than 60 years, the e4 allele was most strongly associated with subjective organization, and to a lesser extent with learning. Subjective organization is an effortful learning strategy requiring an active reorganization of the incoming information to enhance the quality of encoding, and is thought to be mediated by the prefrontal lobe (Fletcher, Shallice, \& Dolan, 1998; Fletcher et al., 1998; Kramer et al., 2005; Savage et al., 2001). In order to investigate whether this effect was mediated by impairment in executive function, we post hoc repeated the analyses with correction for Stroop card III score and TMT B score (Reitan, 1958; Stroop, 1935). In these analyses, subjective organization remains significantly related to APOE genotype, which suggests that subjective organization problems in APOE e4 carriers can not be explained by impairments in executive functioning. No other studies have investigated the effect of APOE on memory in middle-aged subjects with $\mathrm{MCl}$, but in healthy young and middle-aged subjects the APOE e4 allele did not have an effect on memory function (Jorm et al., 2007). The lack of effect in young and middle-aged healthy APOE e4 carriers could be explained by test selection, because only measures of learning or delayed recall were tested which was not severely affected by the APOE e4 allele in our study. Alternatively, the effect of the APOE e4 allele on cognitive function in younger subjects may become apparent only if impairment is severe enough to meet criteria of $\mathrm{MCl}$ and leads to referral to a memory clinic.

The effect of the APOE e4 allele on memory functioning in the middle-aged subgroup seemed not to be modulated by incipient $A D$, because after exclusion of subjects who converted to $A D$ subjective organization remained associated with the e4 allele. This could mean that the APOE e4 allele directly affects memory functioning independently of AD related pathology, for example by its effect on lipid or glucose metabolism (Martins et al., 2006). Another explanation is that the memory problems were related to AD pathology, but that in these middle-aged subjects the follow-up interval was not long enough to identify everyone who would progress to clinical AD. In that case, subjective organization could be a very early marker of AD (Carlesimo et al., 1998; Delis et al., 1991; Perri et al., 2005). 
There is ample evidence that the APOE e4 allele affects brain functioning in parietal, temporal and prefrontal regions in young to middle-aged subjects who are cognitively intact (Reiman et al., 2004; Scarmeas et al., 2003; Trivedi et al., 2006). The interpretation of these studies is still controversial. Some studies suggest that these brain abnormalities reflect early $A D$ as $A D$ pathology is found in the same regions and may be present decades before the onset of dementia (Mondadori et al., 2006; Reiman et al., 2004). Others consider this unlikely and suggest that these abnormalities are a direct effect of the APOE genotype on lipid or glucose metabolism that may facilitate the onset of AD at a later age (Scarmeas et al., 2005). The effect of APOE on frontal lobe functioning in young to middle-aged subjects may explain the effect of APOE on subjective organization in the present study (Bartzokis et al., 2006; Bookheimer et al., 2000; Filbey et al., 2006; Reiman et al., 2004; Wishart et al., 2006). Although neuroimaging studies in this age range showed also impairments in the medial temporal lobe, learning and delayed recall were not severely affected in the middle-aged subgroup in our study (Bookheimer et al., 2000; Scarmeas et al., 2005; Wishart et al., 2006). One explanation is that these memory functions could still be compensated for.

Serial clustering, primacy, recency, intrusions and retention were not related to APOE genotype. Serial clustering, primacy, recency do not require active organization of the incoming information and are more related to working memory and short term memory processes. In general, these aspects of memory functioning are relatively less sensitive to neurodegeneration (Perri et al., 2005). The lack of an effect of the number of intrusions might be explained by the low prevalence of intrusions in the total sample. Retention was lower in APOE e4 carriers, but did not reach statistical significance.

The APOE allele distribution in our sample is comparable with that reported in other studies with MCI (Farlow et al., 2004; Petersen et al., 1995). The prevalence of the APOE e4 allele in our middle-aged subgroup (40\%) and in the elderly subgroup (58\%) was higher than the prevalence of the APOE e4 allele in subjects from the general population aged $40-60(32 \%)$ and 60-85 years (24\%) from the Maastricht Aging Study (MAAS) (Jolles et al., 1995). This suggests that carriers of the APOE e4 allele more often have memory problems leading to a referral to a memory clinic than non-carriers, even at a relative young age.

Strengths of the present study include the large sample size, the broad age range, the variety of memory measures tested, and the long follow-up period to identify subjects with incident AD. The study also had some limitations. Firstly, after exclusion of those who developed $A D$ the sample size in the older subgroup was small, resulting in only 10 subjects older than 60 years who carried an e4 allele. Secondly, because subjects were selected from a memory clinic results may not be generalizable to other settings and subjects with $\mathrm{MCl}$ according to other definitions. Thirdly, the follow-up period may be too short to identify all subjects who convert 
to AD. Another point is that blood samples for APOE genotyping were collected at follow-up only. However, the baseline characteristics of the subjects with data on APOE genotype and those without data on APOE genotype were comparable, except regarding age, which was 3 years lower in those with genotyping. This suggests that genotyping at follow-up may not have introduced a strong bias.

In conclusion, the findings of this study, that investigated several aspects of verbal memory across a broad age range in clinical subjects with $\mathrm{MCl}$ showed that APOE genotype has an effect on memory that is dependent on age. In subjects of 60 years and older, memory functions associated with the medial temporal lobe were predominantly affected, while in middle-aged subjects memory functioning related to the frontal lobe was most strongly affected. Together with the observation that memory functioning was dependent on $A D$ pathology in elderly subjects, but not in middle-aged subjects, this suggests that different mechanisms underlie the APOE-related memory dysfunction in different age groups. Whether the effect of APOE genotype on subjective organization in middle-aged subjects is indeed an $A D$ independent process needs to be investigated in future imaging studies and longitudinal studies with a longer follow-up. 



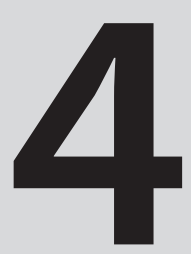

Affective symptoms as predictors for Alzheimer's disease in subjects with Mild Cognitive Impairment: a 10-year followup study

Ramakers, IHGB., Visser, PJ., Aalten, P., Kester, A., Jolles, J. \& Verhey, FRJ. 


\section{Abstract}

Context: Affective symptoms are common in subjects with mild cognitive impairment $(\mathrm{MCl})$. Previous studies yielded conflicting results regarding the question whether these symptoms are predictive for future Alzheimer's disease (AD).

Objectives: To investigate the predictive accuracy of affective symptoms for AD during a 10year follow-up in subject with $\mathrm{MCl}$, and to investigate whether the predictive accuracy was modified by age, the presence of amnestic $\mathrm{MCl}$ and length of follow-up.

Design: Prospective cohort study with follow-up assessments after 2,5 and 10 years.

Setting: Memory clinic

Participants: 263 consecutive newly referred subjects with $\mathrm{MCl}$ older than 55 years and without obvious causes of cognitive impairment.

Assessment: Ratings for depression, anxiety, apathy, and sleeping problems from the Hamilton Depression Rating Scale.

Main Outcome Measure: Conversion to AD at follow-up.

Results: Affective symptoms were present in $50-70 \%$ of the subjects. The average follow-up period was 5.4 years and 79 subjects (29\%) developed AD. All affective symptoms were associated with a decreased risk for $A D$, which was statistically significant for sleeping problems (sleeping problems $\mathrm{OR}=0.35(95 \% \mathrm{Cl}=0.20-0.62, \mathrm{P}<0.001)$, depression $\mathrm{OR}=0.61(95 \% \mathrm{Cl}=0.36-1.01, \mathrm{P}$ $=0.059)$, anxiety $\mathrm{OR}=0.58(95 \% \mathrm{Cl}=0.34-1.0, \mathrm{P}=0.051)$, apathy $\mathrm{OR}=0.67(95 \% \mathrm{Cl}=0.4-1.1$, $P=0.14)$ ). Depression was associated with a decreased risk for $A D$ only in subjects without amnestic $\mathrm{MCl}(\mathrm{OR}=0.34,95 \% \mathrm{Cl}=0.13-0.93, \mathrm{P}=0.036)$, but not in subjects with amnestic $\mathrm{MCl}(\mathrm{OR}=1.1,95 \% \mathrm{Cl}=0.50-2.3, \mathrm{P}=0.85)$. The risk of anxiety for $\mathrm{AD}$ was different $(\mathrm{P}=0.046)$ between subjects diagnosed with $A D$ at the 5 -year follow-up $(O R=0.23,95 \% \mathrm{Cl}=0.0-0.66$, $\mathrm{P}=0.077)$ and subjects diagnosed with $\mathrm{AD}$ at the 10 -year follow-up $(\mathrm{OR}=1.7,95 \% \mathrm{Cl}=0.32$ 9.01, $P=0.539)$.

Conclusions: Affective symptoms are generally associated with a decreased risk for AD. The risk may be dependent on $\mathrm{MCl}$ subtype or length of follow-up, but it does not depend on age.

Key words: Affective symptoms, Mild Cognitive Impairment, Alzheimer's disease, and predictive accuracy. 


\section{Introduction}

Mild Cognitive Impairment $(\mathrm{MCl})$ is a condition that refers to cognitive impairment in subjects without dementia. It has many causes, including Alzheimer's disease (AD) (Visser et al., 2006). It is important to identify $\mathrm{MCl}$ subjects with prodromal $\mathrm{AD}$, because if disease modifying drugs for $A D$ become available, these are likely be most effective in the early stage of the disease. A possible predictor for $A D$ are affective symptoms, such as depression, anxiety and apathy (Ownby et al., 2006). These symptoms are common in subjects with $\mathrm{MCl}$ (Apostolova \& Cummings, 2007), but it is uncertain whether they can predict AD in this population (Steffens et al., 2006). Depressive symptoms were associated with an increased risk for AD in some studies (Gabryelewicz et al., 2007; Modrego \& Ferrandez, 2004), and with a decreased risk in others (Liu et al., 2007; Rozzini et al., 2005), while most studies did not find a relation between depression and AD at all (Table 1) (Copeland et al., 2003; Feldman et al., 2007; Korf et al., 2004; Palmer et al., 2007; Panza et al., 2008; Robert et al., 2006; Teng et al., 2007; Tierney et al., 1999; Visser et al., 2000; Visser et al., 2000; Wang et al., 2006). Similarly, discrepant results have been reported regarding the predictive accuracy of apathy and anxiety for AD (Feldman et al., 2007; Liu et al., 2007; Palmer et al., 2007; Robert et al., 2006; Teng et al., 2007).

The contradictory findings suggest that the relation between affective symptoms and $A D$ is modified by other factors, which varied between studies, but these factors have not yet been identified. Statistically non-significant findings may be explained by a small sample size or a brief follow-up period. Aim of the present study was to investigate the predictive accuracy of affective symptoms for $\mathrm{AD}$ in a large clinical cohort of subjects with $\mathrm{MCl}$ over a 10-year period. In addition, we investigated whether age, the presence of amnestic $\mathrm{MCl}$, and length of follow-up modified the relation between affective symptoms and AD. We hypothesized that the association between affective symptoms and $A D$ would be strongest in elderly subjects or in subjects with amnestic $\mathrm{MCl}$, as these subjects are more likely to develop AD (Visser et al., 2006). We investigated length of follow-up as possible effect modificator, because previous studies suggested that the risk of depression for dementia may increase or decrease with length of follow-up (Green et al., 2003; Ownby et al., 2006). Besides depression, anxiety, and apathy, we also investigated sleeping problems as a predictor for AD. Sleeping problems are common in affective disorders and previous studies found that these problems may be predictive for cognitive decline or dementia (Jelicic et al., 2002). 
Table 1: Studies on the predictive value of depressive symptoms for AD or dementia in subjects with $\mathrm{MCl}$.

\begin{tabular}{|c|c|c|c|c|c|c|c|}
\hline Study & Setting & $\mathrm{N}$ & MCl-definition & Age & MMSE & $\begin{array}{l}\text { Depression } \\
\text { scale }\end{array}$ & Depression diagnosis \\
\hline Tierney et al., 1999 & Community & 143 & GDS 2 or 3 & 72.0 & $27.8^{*}$ & GDS-30 & GDS-30 $>10$ \\
\hline Visser et al., 2000 & Clinical & 74 & $\mathrm{aMCl}$ & 61.1 & 27.3 & HDRS & $\begin{array}{l}\text { DSM IV minor and major depression, } \\
\text { and HDRS > } 13\end{array}$ \\
\hline Visser et al., 2000 & Clinical & 111 & GDS 2 or 3 & 66.0 & 27.9 & HDRS & DSM IV minor and major depression \\
\hline Visser et al., 2002 & Community & 45 & $\begin{array}{l}\text { Minimal } \\
\text { dementia }\end{array}$ & 79.4 & 22.8 & na & CAMDEX-criteria \\
\hline $\begin{array}{l}\text { Copeland et al., } \\
2003\end{array}$ & Community & 123 & $\mathrm{CDR}=0.5$ & 72.0 & 29.2 & na & $\begin{array}{l}\text { at least } 1 \text { symptom DSM-III-R depression } \\
\text { criteria }\end{array}$ \\
\hline $\begin{array}{l}\text { Estévez-González et } \\
\text { al., } 2003\end{array}$ & Clinical & 70 & GDS 2 or 3 & 68.1 & 27.5 & GDS-30 & na \\
\hline Tian et al., 2003 & Clinical & 129 & Test impairment & 70.0 & 24.6 & Cornell & na \\
\hline Korf et al., 2004 & Clinical & 75 & Test impairment & 62.8 & 26.9 & MADRS & MADRS $>6$ \\
\hline $\begin{array}{l}\text { Modrego \& } \\
\text { Ferrandez, } 2004\end{array}$ & Clinical & 114 & $\mathrm{aMCl}$ & 72.8 & 27.8 & GDS-30 & $\begin{array}{l}\text { GDS-30 }>9 \text { and DSM-IV depression } \\
\text { or depressed mood without guild/ } \\
\text { worthlessness }\end{array}$ \\
\hline Rozzini et al., 2005 & Clinical & 46 & $\mathrm{aMCl}$ & 70.5 & 26.8 & GDS-15 & DSM-IV major depression \\
\hline Robert et al., 2006 & Clinical & 216 & $\begin{array}{l}\text { Complaints and } \\
\text { test impairment }\end{array}$ & 71.9 & 27.5 & MADRS & MADRS $>10$ \\
\hline Wang et al., 2006 & Clinical & 58 & $\mathrm{aMCl}$ & 76.3 & 25.9 & GDS-15 & na \\
\hline Yaffe et al., 2006 & Clinical & 327 & Test impairment & 72.9 & 25.7 & na & $\begin{array}{l}\text { At least } 2 \text { symptoms DSM-III-R } \\
\text { depression criteria }\end{array}$ \\
\hline Feldman et al., 2007 & Clinical & 510 & $\begin{array}{l}\text { CDR } 0.5 \text { and } \\
\mathrm{aMCl}\end{array}$ & 70.6 & 26.9 & HDRS & na \\
\hline $\begin{array}{l}\text { Gabrylewicz et al., } \\
2007\end{array}$ & Clinical & 105 & $\begin{array}{l}\text { CDR } 0.5 \text { and test } \\
\text { impairment }\end{array}$ & 69.3 & 27.2 & MADRS & na \\
\hline Liu et al., 2007 & Clinical & 124 & CDR 0.5 & 74.7 & 25.2 & HDRS & na \\
\hline Palmer et al., 2007 & Community & 47 & $\begin{array}{l}\text { Complaints and } \\
\text { test impairment }\end{array}$ & 84.0 & na & CPRS mood & CPRS mood $>1$ \\
\hline Teng et al., 2007 & Clinical & 51 & $\begin{array}{l}\text { Complaints and } \\
\text { test impairment }\end{array}$ & 72.8 & 27.6 & $\begin{array}{l}\text { NPI- } \\
\text { depression }\end{array}$ & NPI-depression score $>0$ \\
\hline Panza et al., 2008 & Community & 139 & Test impairment & 80.6 & 21.4 & GDS-30 & GDS-30 $>9$ \\
\hline
\end{tabular}


Table 1 continued: Studies on the predictive value of depressive symptoms for AD or dementia in subjects with $\mathrm{MCl}$.

\begin{tabular}{|c|c|c|c|c|c|c|c|c|}
\hline \multirow[t]{2}{*}{ Study } & \multirow{2}{*}{$\begin{array}{l}\text { Exclusion criteria } \\
\text { depression }\end{array}$} & \multirow{2}{*}{$\begin{array}{l}\text { Prevalence } \\
\text { depression }\end{array}$} & \multirow{2}{*}{$\begin{array}{l}\text { Depression } \\
\text { scale score }\end{array}$} & \multirow{2}{*}{$\begin{array}{l}\text { Years follow } \\
\text { up }\end{array}$} & \multirow[t]{2}{*}{ Outcome } & \multicolumn{3}{|c|}{ Prediction } \\
\hline & & & & & & $\begin{array}{l}\text { Relation with } \\
\text { outcome\# }\end{array}$ & $\mathrm{ES} \wedge(95 \% \mathrm{Cl})$ & $\mathrm{OR} \wedge \wedge(95 \% \mathrm{Cl})$ \\
\hline Tierney et al., 1999 & None & $22 \%$ & 4.9 & 2 & $A D$ & 0 & $-0.04(0.47-0.39)$ & $0.99(0.35-2.8)$ \\
\hline Visser et al., 2000 & None & $35 \%$ & 11.1 & 3.7 & $A D$ & 0 & $-0.33(-0.87-0.21)$ & $0.57(0.17-1.9)$ \\
\hline Visser et al., 2000 & None & $56 \%$ & 10.1 & 2.6 & Dementia & 0 & $-0.14(-0.59-0.32)$ & $1.38(0.55-3.5)$ \\
\hline Visser et al., 2002 & None & $13 \%$ & na & 2.3 & Dementia & 0 & na & $1.2(0.24-6.0)$ \\
\hline $\begin{array}{l}\text { Copeland et al., } \\
2003\end{array}$ & None & $38 \%$ & na & 3 & $A D$ & 0 & $0.10(-0.37-0.56)$ & $1.09(0.43-2.8)$ \\
\hline $\begin{array}{l}\text { Estévez-González et } \\
\text { al., } 2003\end{array}$ & None & na & 11.2 & 2.3 & $A D$ & na & $-0.56(-1.1-0.07)$ & na \\
\hline Tian et al., 2003 & None & na & 4.3 & 2 & Dementia & 0 & $-0.03(-0.41-0.36)$ & na \\
\hline Korf et al., 2004 & None & $42 \%$ & na & 2.8 & Dementia & 0 & na & $0.48(0.2-1.2)$ \\
\hline $\begin{array}{l}\text { Modrego \& } \\
\text { Ferrandez, } 2004\end{array}$ & None & $36 \%$ & 9.6 & 3 & $A D$ & + & $1.25(0.84-1.65)$ & $11.9(4.4-32.2)$ \\
\hline Rozzini et al., 2005 & None & $48 \%$ & na & 2 & $A D$ & - & na & $0.24(0.07-0.81)$ \\
\hline Robert et al., 2006 & MADRS $>20$ & $20 \%$ & 6.9 & 1 & $A D$ & 0 & $0.28(-0.16-0.72)$ & $2.1(0.8-5.4)$ \\
\hline Wang et al., 2006 & None & na & 4.0 & 1.8 & $A D$ & 0 & $-0.46(-1.0-0.09)$ & na \\
\hline Yaffe et al., 2006 & na & $38 \%$ & na & 3.1 & Dementia & 0 & na & $1.23(0.75-2.0)$ \\
\hline Feldman et al., 2007 & $\begin{array}{l}\text { HDRS }>12 \text { and } \\
\text { HDRS item } 1>0\end{array}$ & na & 2.7 & 4 & $A D$ & 0 & $0.04(-0.17-0.25)$ & na \\
\hline $\begin{array}{l}\text { Gabrylewicz et al., } \\
2007\end{array}$ & MADRS $>28$ & na & 9.7 & 3 & Dementia & + & $0.32(-0.15-0.78)$ & na \\
\hline Liu et al., 2007 & None & na & 8.2 & 1.7 & $A D$ & - & $-0.49(-0.88-0.09)$ & na \\
\hline Palmer et al., 2007 & None & $36 \%$ & na & 3 & $A D$ & 0 & na & $1.3(0.4-5.1)$ \\
\hline Teng et al., 2007 & None & $39 \%$ & 1.2 & 2 & $A D$ & + & na & $4.5(1.1-17.9)$ \\
\hline Panza et al., 2008 & None & $61 \%$ & na & 3.5 & Dementia & 0 & na & $1.6(0.8-3.1)$ \\
\hline
\end{tabular}

legend on page 54 


\section{Methods}

\section{Subjects}

Subjects were selected from an ongoing longitudinal study of non-demented subjects referred to the Maastricht Memory Clinic. The memory clinic is an outpatient clinic of the Maastricht University hospital (Verhey et al., 1993). The design of this study has been described in detail elsewhere (Verhey et al., 1993; Visser et al., 2006). Consecutive patients were included at the time of the first visit to the memory clinic. Subjects included had to be 40 years or older and to have $\mathrm{MCl}$, which was defined as a score of 2 or 3 on the Global Deterioration Scale (GDS) (Reisberg et al., 1982). This broad definition of $\mathrm{MCl}$ is consistent with that of other studies (Feldman \& Jacova, 2005; Tabert et al., 2006; Tierney et al., 1996). This definition was used because subjects with $\mathrm{AD}$ in the predementia stage often do not meet the more strict criteria of $\mathrm{MCl}$, such as the criteria for aMCI (Dickerson et al., 2007; Geslani et al., 2005; Storandt et al., 2006; Visser \& Verhey, 2007). Exclusion criteria were the existence of dementia at baseline and an apparent cause for cognitive impairment, such as cerebrovascular disorders, brain trauma, endocrine disorders or psychiatric disorders other than mild affective disorders at baseline (Visser et al., 2000).

For the present study we selected subjects older than 55 years who had been eligible for at least one follow-up assessment $(n=263)$. Subjects included in this study were referred to the memory clinic by general practitioners (72\%), neurologists (4\%), psychiatrists (13\%) or

\section{Legend Table 1}

na = not available, MCI: Mild Cognitive Impairment, aMCI: amnestic Mild Cognitive Impairment, CIND: Cognitive Impairment No Dementia, AD: Alzheimer disease, HDRS: Hamilton Depression Rating Scale, GDS-15: Geriatric Depression Scale 15 items, GDS-30: Geriatric Depression Scale 30 items, GDS: Global Deterioration Scale, NPI: Neuropsychiatric Inventory, CPRS: Comprehensive Psychopathological Rating Scale, GMSS: Geriatric Mental State Schedule, CESDS: Center for Epidemiologic Studies Depression Scale, mCESDS: modified Center for Epidemiological Studies Depression Scale, CDR: Clinical Dementia Rating, Cornell: Cornell Scale for Depression in Dementia.

\# This column summarizes statistically significant findings as reported in each study. $0=$ depression is not associated with outcome; $+=$ depression is associated with an increased risk for AD or dementia; - = depression is associated with a decreased risk for AD or dementia.

$\wedge \mathrm{ES}=$ Effect Size based on differences in depression scores between subjects with and without $A D$ or dementia at follow-up and presented as Cohen's delta. A negative score means that subjects with AD or dementia at follow-up have a lower score on the depression scale than subjects without AD or dementia at follow-up.

$\wedge \wedge \mathrm{OR}=$ Odds ratio. Odds ratio was based on dichotomous data presented in the publication and uncorrected for any confounders. An OR below 1 indicates that depression is associated with a lower risk for AD or dementia at follow-up.

* MMSE based on data from another study, which included a similar sample (Tierney et al., 1996). 
others (11\%). The study was approved by the medical ethical Committee at the Maastricht University hospital. After the study was explained to the subjects, all subjects gave their written informed consent.

\section{Clinical assessment at baseline and follow-up}

At baseline, all subjects underwent a standardised assessment, which included a detailed history of the subject, a psychiatric, neurological, and physical examination, the Mini Mental State Examination (MMSE) (Folstein et al., 1975), the GDS (Reisberg et al., 1982), the 17-item version of the Hamilton Depression Rating Scale (HDRS) (Hamilton, 1960), the Blessed Dementia Rating Scale (BDS) (Blessed et al., 1968), and appropriate laboratory tests, a neuropsychological assessment including tests for measuring learning and memory, working memory, fluency, intelligence, speed of information processing and executive functioning, and a CT or MRI as described elsewhere (Verhey et al., 1993).

Two, five and ten years after baseline, subjects were invited to participate in a follow-up assessment. Because subjects were continuously enrolled, not all subjects were long enough in the study to have a follow-up assessment after 5 or 10 -years. At the time of analyses, 263 subjects had been eligible for the two-year follow-up, 232 for the five-year followup and 133 for the ten-year follow-up. The follow-up assessment included a standardized questionnaire about medical history and cognitive complaints, the MMSE, GDS, BDS, HDRS, and a neuropsychological assessment comparable to the baseline assessment. Subjects who refused to come for the follow-up assessment were contacted via a telephone interview, which included a standardized questionnaire about medical history and cognitive complaints, and the Telephone Interview for Cognitive Status (Brandt et al., 1988).

Amnestic $\mathrm{MCl}$ was defined as a score 1.5 standard deviation (SD) below the mean of a reference population, after correction for age, sex and education on the delayed recall measure of the Auditory Verbal Learning Test (AVLT) (Petersen et al., 1999) (Van der Elst et al., 2005). The amnestic $\mathrm{MCl}$ criteria also require also intact activities of daily living. This was operationalized as the absence of dementia and a score below 4 on the GDS, which was part of the inclusion criteria of the study, in accordance with previous studies (Geslani et al., 2005).

The diagnosis of dementia and AD was made according to the DSM-IV and NINCDSADRDA criteria (APA, 1994; McKhann et al., 1984). The diagnosis of frontotemporal dementia was made according to the Neary criteria (Neary et al., 1998). Vascular dementia was diagnosed according to the NINDS-AIREN criteria (Roman et al., 1993). Diagnoses of dementia and AD were made independently by both a neuropsychiatrist and a neuropsychologist. Both were blinded to the baseline results. If disagreement on the diagnosis occured, a consensus meeting was held. If no consensus could be reached, the subject was considered not demented. 
Table 2: Baseline comparisons of the converters and non-converters.

\begin{tabular}{|c|c|c|c|c|c|}
\hline & \multirow{2}{*}{$\begin{array}{l}\text { Total group } \\
(n=263)\end{array}$} & \multicolumn{3}{|c|}{ Outcome at follow-up } & \multirow{2}{*}{$\begin{array}{l}\text { PValue not } \\
\text { demented } \\
\text { vs. AD }\end{array}$} \\
\hline & & $\begin{array}{l}\text { Not } \\
\text { demented } \\
(n=125)\end{array}$ & $\begin{array}{l}A D \\
(n=79)\end{array}$ & $\begin{array}{l}\text { Other } \\
\text { dementia } \\
(n=11)\end{array}$ & \\
\hline Age $(M \pm S D)$ & $66.9 \pm 7.7$ & $64.2 \pm 7.4$ & $69.8 \pm 6.8$ & $65.8 \pm 5.1$ & .000 \\
\hline Older than 70 years (\%) & 36 & 25 & 46 & 18 & .003 \\
\hline Sex (\% female) & 44 & 39 & 54 & 27 & .024 \\
\hline Education (\% low-middle-high) & $23 / 46 / 31$ & 19/50/31 & $23 / 45 / 32$ & $18 / 46 / 36$ & .747 \\
\hline MMSE $(M \pm S D)$ & $27.6 \pm 2.1$ & $28.3 \pm 1.6$ & $26.3 \pm 2.3$ & $27.7 \pm 1.6$ & .000 \\
\hline AVLT-delayed recall $(\mathrm{M} \pm \mathrm{SD})$ & $6.0 \pm 3.8$ & $7.7 \pm 3.2$ & $2.8 \pm 2.5$ & $7.6 \pm 2.1$ & .000 \\
\hline Amnestic $\mathrm{MCl}(\%)$ & 42 & 22 & 77 & 18 & .000 \\
\hline HDRS-total score $(\mathrm{M} \pm \mathrm{SD})$ & $9.0 \pm 6.2$ & $9.1 \pm 6.4$ & $7.7 \pm 5.7$ & $10.6 \pm 6.0$ & .110 \\
\hline Major depression (\%) & 21 & 22 & 13 & 18 & .124 \\
\hline BDS-total score $(\mathrm{M} \pm \mathrm{SD})$ & $1.8 \pm 1.8$ & $1.3 \pm 1.4$ & $1.9 \pm 1.6$ & $3.3 \pm 4.0$ & .004 \\
\hline GDS-total score $(\mathrm{M} \pm \mathrm{SD})$ & $2.5 \pm 0.5$ & $2.3 \pm 0.5$ & $2.7 \pm 0.5$ & $2.6 \pm 0.5$ & .000 \\
\hline \multicolumn{6}{|l|}{ Affective symptoms (\%) } \\
\hline Depression & 53 & 56 & 43 & 64 & .078 \\
\hline Anxiety & 54 & 54 & 50 & 50 & .583 \\
\hline Apathy & 65 & 68 & 53 & 73 & .038 \\
\hline Sleeping problems & 50 & 56 & 37 & 27 & .008 \\
\hline
\end{tabular}

$\mathrm{M}=$ mean, $\mathrm{SD}=$ Standard deviation, $\mathrm{MMSE}=$ Mini Mental State Examination, AVLT = Auditory Verbal Learning Test, HDRS = Hamilton Depression Rating Scale, BDS = Blessed Dementia Rating Scale, GDS = Global Deterioration Scale.

\section{Affective symptoms}

From the HDRS (Hamilton, 1960) we used the subscales for anxiety (item 9, 10, 11, 15, and 17), depression (item 1, 2, 3, 7, and 8), insomnia (item 4, 5, and 6) (Shafer, 2006), and apathy (item 7, 8, and 13) (Li et al., 2001).

\section{Statistical analyses}

Statistical analyses were performed using the Statistical Package for Social Sciences (SPSS), version 11 for Mac OS X. We dichotomized all affective symptom scores. Symptoms were considered to be clinically present if at least one item included in a subscale was either one or 
higher for items scored on a 3-point scale, or was 2 or higher for items scored on a 5-point scale. Group differences were analyzed using an independent t-test or Chi square test. Predictive accuracy was analyzed using a discrete-time survival analysis model and implemented using logistic regression and an appropriately adjusted dataset (Hosmer \& Lemeshow, 1999). The outcome group included all subjects with $A D$ at follow-up. The reference group included subjects without dementia or with non-AD dementia at follow-up. Analyses were performed with and without adjustment for age, sex, and education. The difference in OR for AD between subjects diagnosed with $A D$ at the 2-year, 5-year and 10-year follow-up was tested using the discrete-time survival analysis model (Hosmer \& Lemeshow, 1999). We also investigated interactions of affective symptoms with amnestic $\mathrm{MCl}$, and age (younger or older than 70 years). In order to investigate whether findings were influenced by the inclusion of subjects with non-AD type dementia in the reference group, we repeated analyses after exclusion of subjects with non-AD type dementia.

\section{Results}

At baseline, $263 \mathrm{MCl}$ subjects were included in the study. Their baseline characteristics are listed in Table 2. Symptoms of depression, anxiety, and sleeping problems were present in about $50 \%$ of the subjects, and symptoms of apathy in about $70 \%$ (Table 2 ).

\section{Outcome at follow-up}

At least one follow-up was available for 228 subjects (87\%). The number of subjects at each follow-up and the reason for the absence of a follow-up are shown in Figure 1. Subjects without outcome at a follow-up for which they had been eligible were similar to subjects with outcome with regard to: age, sex, education, MMSE score, score on the delayed recall test, HDRS score, BDS score, proportion of subjects with amnestic MCI and GDS score.

Seventy-nine subjects developed $A D$ and 11 subjects other types of dementia (vascular dementia $(n=6)$, frontotemporal dementia $(n=1)$, Parkinson dementia $(n=3)$ and primary progressive aphasia $(n=1)$ ) at follow-up (see Figure 1 ). The annualized conversion rate to $A D$ was highest for the first years of follow-up and decreased with longer follow-up intervals ( $11 \%$ for the first two years, $4.2 \%$ for year 3 to 5 , and 3.0\% for year 6 to 10). The average followup length was 5.4 years. The baseline characteristics according to outcome are listed in Table 2. Subjects who converted to AD were at baseline about 5 years older, more often female, had lower MMSE and AVLT delayed recall scores, had more often amnestic MCl, and had higher BDS and GDS scores compared to the subjects who did not develop AD (Table 2). 


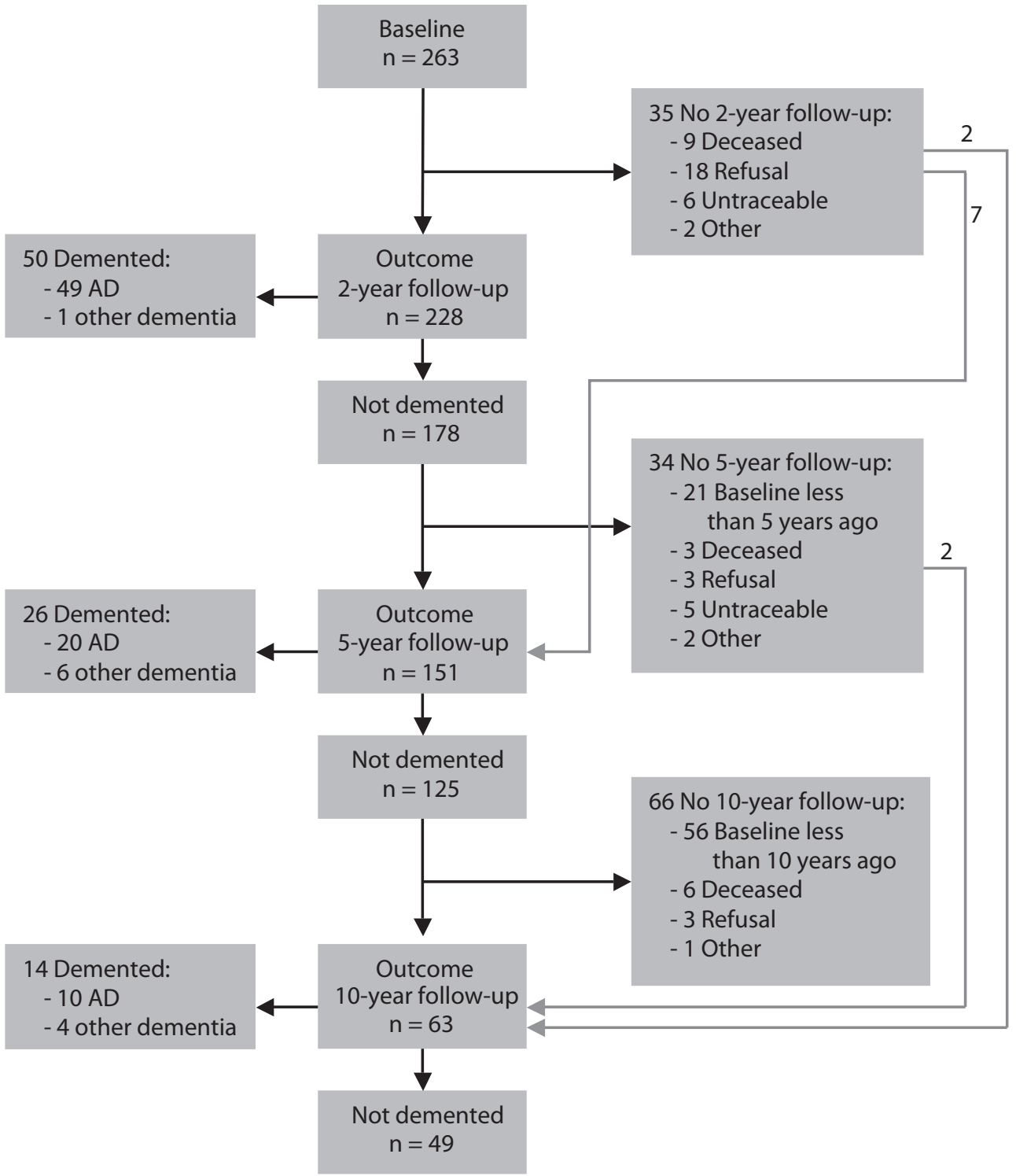

Figure 1: Flowchart of the follow-up 


\section{Predictors for AD}

Univariate analyses showed that the presence of sleeping problems $(\mathrm{OR}=0.43,95 \% \mathrm{Cl}=0.26$ $-0.71, \mathrm{P}=0.001)$, and symptoms of apathy $(\mathrm{OR}=0.58,95 \% \mathrm{Cl}=0.35-0.96, \mathrm{P}=0.034)$ were associated with a decreased risk for $A D$. Depressive symptoms $(O R=0.62,95 \% \mathrm{Cl}=0.38-1.03$, $P=0.065)$ showed a trend in the same direction.

After adjustment for age, sex and education, sleeping problems were associated with a lower risk for $\mathrm{AD}(\mathrm{OR}=0.35,95 \% \mathrm{Cl}=0.20-0.62, \mathrm{P}<0.001)$ (Table 3). Symptoms of depression $(\mathrm{OR}=0.61,95 \% \mathrm{Cl}=0.36-1.01, \mathrm{P}=0.059)$ and anxiety $(\mathrm{OR}=0.58,95 \% \mathrm{Cl}=0.34-1.0, \mathrm{P}=0.051)$ showed a trend in the same direction (Table 3 ). After the exclusion of subjects who developed a non-AD type dementia, results remained essentially the same.

\section{Effect of amnestic $\mathrm{MCl}$ and age}

The presence of amnestic $\mathrm{MCl}$ modified the relation between depressive symptoms and the risk for $A D(P=0.017)$. Depression was associated with a lower risk for $A D$ in subjects without amnestic $\mathrm{MCl}(\mathrm{OR}=0.34,95 \% \mathrm{Cl}=0.13-0.93, \mathrm{P}=0.036)$, but not in subjects with amnestic $\mathrm{MCl}$ $(\mathrm{OR}=1.1,95 \% \mathrm{Cl}=0.50-2.3, \mathrm{P}=0.85)$. Age did not modify the risk for AD for any symptom.

\section{Effect of length of follow-up}

We noted some differences in predictive accuracy according to time to follow-up (Table 3), but these differences were small. Only the risk of anxiety for the development of AD was statistically significantly different $(P=0.046)$ for subjects with $A D$ diagnosed at the 5 -year follow-up (OR $=0.23,95 \% \mathrm{Cl}=0.08-0.66, \mathrm{P}=0.007)$ and subjects newly diagnosed with $\mathrm{AD}$ at the 10 -year follow-up $(\mathrm{OR}=1.7,95 \% \mathrm{Cl}=0.32-9.01, \mathrm{P}=0.539)$.

\section{Comment}

The major findings of this clinical, long-term follow-up study are that sleeping problems were associated with a decreased risk for AD. In addition, symptoms of depression and anxiety showed a trend in this direction. The predictive accuracy of depression was dependent on the presence of amnestic $\mathrm{MCl}$ and that of anxiety on the length of follow-up.

Previous studies on the predictive accuracy of affective symptoms for AD in subjects with $\mathrm{MCl}$ mainly focused on depressive symptoms. The outcomes of our study are consistent with some of these studies (Liu et al., 2007; Rozzini et al., 2005) but in disagreement with others (Table 1) (Gabryelewicz et al., 2007; Modrego \& Ferrandez, 2004). We found that depressive symptoms were only associated with a reduced risk in subjects without amnestic $\mathrm{MCl}$. This corroborates our earlier finding that depression in the absence of moderately severe memory 


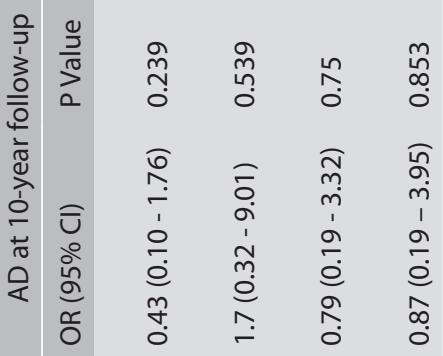

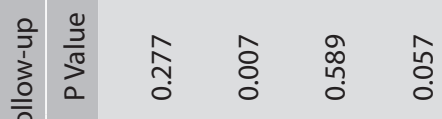

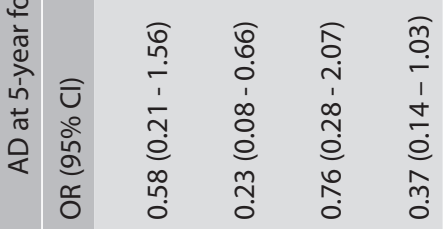

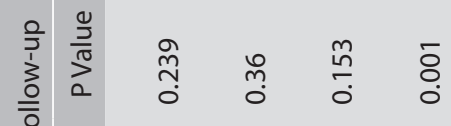

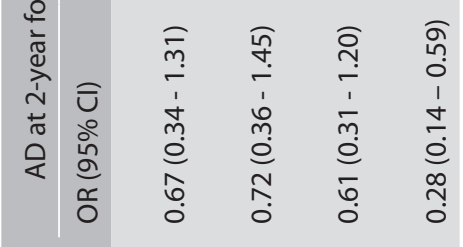

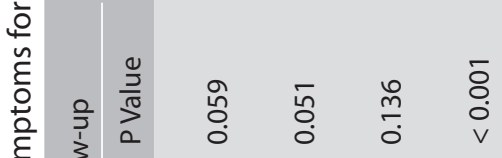

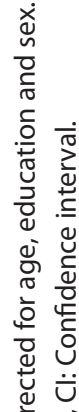

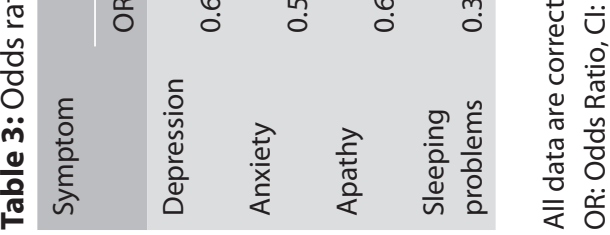


impairment is more likely associated with primary depression than with prodromal AD (Visser, Verhey, Ponds, Kester et al., 2000). It is also in line with the observation that depressed subjects without a plasma biomarker profile suggestive of AD have better memory performances than depressed subjects with such profile (Sun et al., 2008). We did not find that the risk of depressive symptoms for $A D$ was dependent on follow-up length although population-based studies did show that this risk for $A D$ for those with depressive symptoms either increased or decreased with follow-up length (Green et al., 2003; Ownby et al., 2006). If data from studies that provided dichotomous data in Table 1 were pooled with a random effects model (13 studies with 1362 subjects), the OR of depression for AD was 1.31 ( $95 \% \mathrm{Cl}=0.8-2.15, P=0.65$ ) (Deeks et al., 2001). The pooled effect-size based on differences in scores on a depression rating scale expressed as Cohen's delta was $0.0(95 \% \mathrm{Cl}=-0.26-0.25, P=0.98$, data from 1781 subjects from 12 studies). Thus, our pooled data indicate that depression is not predictive for $A D$ in subjects with $\mathrm{MCl}$, unlike a meta-analysis of case-control studies and prospective or retrospective cohort studies of subjects who were not selected on the basis of the presence of $\mathrm{MCl}$, which found that depression was associated with an increased risk for $A D$ or dementia $(O R=2.03,95 \% \mathrm{Cl}=$ 1.81-2.28) (Ownby et al., 2006). Apparently, the relation between depressive symptoms and subsequent cognitive decline is less strong in subjects with $\mathrm{MCl}$ than in subjects unselected for the presence of $\mathrm{MCl}$. One possible explanation for this difference is that the inclusion criteria for $\mathrm{MCl}$ may be biased toward inclusion of subjects that have depression-related cognitive impairment. Support for this explanation comes from the observation that many depressed subjects complain about cognitive impairment and often have cognitive impairment on neuropsychological tests and for that reason may meet the criteria of MCI (Kahn et al., 1975; La Rue et al., 1986).

Anxiety was found to be associated with a decreased risk for AD in two other studies as well (Liu et al., 2007; Robert et al., 2006). but a population-based study reached the opposite conclusion (Palmer et al., 2007). These conflicting findings may be explained by a difference in setting. Our findings suggested that anxiety is associated with a decreased risk in the short time and an increased risk in the long term. This may indicate that the risk of anxiety for AD is dependent in the length of follow-up.

Unlike our study, previous studies reported that apathy was associated with an increased risk for $A D$, although this reached statistical significance in only one study (Feldman et al., 2007; Robert et al., 2006; Teng et al., 2007). These conflicting findings may have resulted from different ways of assessing these symptoms, as we used a subscale from the HDRS, while other studies used the informant based Neuropsychiatric Inventory (Cummings et al., 1994).

Sleeping problems have not been investigated as predictors for AD before in subjects with $\mathrm{MCl}$. While it is well known that sleep disturbances occur in mild and moderate phases of 
$A D$, the present data show that they are not associated with an increased risk for AD in subjects with $\mathrm{MCl}$. In contrast to our findings, a previous study conducted in the general population showed that sleep problems were associated with cognitive decline (Jelicic et al., 2002), although other studies did not find this association (Foley et al., 2001; Tworoger et al., 2006).

Age did not modify the relation between affective symptoms and AD, although we expected that affective symptoms would be associated with an increased risk for AD in elderly subjects. It is possible that the age range in our study was not large enough to detect such an interaction.

There are a number of factors not investigated in the present study that may explain the conflicting results between studies. First, we already mentioned that predictive accuracy may depend on whether the study is conducted in the community or in a clinical setting. In addition, the accuracy may depend on how subjects are selected. For example, due to the referral process, our sample may have been relatively enriched by subjects with primary depression, which would explain the high prevalence of depressive symptoms and the decreased risk of affective symptoms for AD. Secondly, predictive accuracy may depend on the type of rating scale used. We used a clinician based rating scale for affective symptoms, like most of the other studies. Using a self-rating scale for depression and anxiety (Arrindell \& Ettema, 2003; Arrindell \& Ettema, 1981) would have yielded similar results (data not shown). Also if we used a diagnosis of major depression as predictor, we would obtain similar results $(O R=0.58$, $95 \% \mathrm{Cl} 0.29-1.2, \mathrm{P}=0.13)$. Thirdly, the variability between the studies may be explained by differences in the presence of vascular disorders or vascular risk factors, because these factors may modify the relation between depression and cognitive impairment (Alexopoulos, 2006). Fourthly, the variability may have resulted from an interaction between age, $\mathrm{MCl}$ type, length of follow-up, setting, vascular risk factors, and diagnostic instruments. It is also possible that the conflicting findings reflect random variation given the observation that the pooled OR of depressive symptoms for AD was close to 1 and not statistically significant.

The annual conversion ratio of $11 \%$ for the first two years is comparable with the mean annual conversion rate of $10.2 \%$, found in a meta-analysis (Bruscoli \& Lovestone, 2004). The annual conversion ratio in our study declined with the length of follow-up as described before (Visser et al., 2006).

Few subjects developed non-AD types of dementia, which is in line with other clinical $\mathrm{MCl}$ studies (Geslani et al., 2005). After exclusion of subjects with non-AD types of dementia results remained similar, indicating that the inclusion of subjects with a non-AD type dementia in the reference group did not have effect on the results.

Strengths of the present study were: the large sample size, the long follow-up period of up to 10 years, the high follow-up rate, and the broad inclusion criteria for $\mathrm{MCl}$ in which 
mild depressive symptomatology was not excluded at baseline, in accordance with current recommendations (Steffens et al., 2006). The limitations of the study were the relatively small number of subjects with a 10-year follow-up and the lack of pathological confirmation of the clinical diagnosis.

There have been several hypotheses regarding the relation between depression, $\mathrm{MCl}$ and AD (Steffens et al., 2006; Wilson et al., 2008). One hypothesis proposes that depressive symptoms contribute to the development of AD. A second hypothesis suggests that depressive symptoms may be an early manifestation of $A D$. A third hypothesis is that the cognitive impairment results from a primary depressive disorder. Our data supports the last hypothesis as we found that affective symptoms were generally associated with a decreased risk for AD.

The present study indicates that affective symptoms in subjects with $\mathrm{MCl}$ are not predictive for prodromal AD. Given the high prevalence of affective symptoms in prodromal $A D(37-53 \%)$, however, the presence of these symptoms does certainly not exclude the possibility of prodromal AD. Therefore, mild affective symptoms should not be an exclusion criteria for studies that aim to select these subjects, as has been the case in previous $\mathrm{MCl}$ trials (Visser et al., 2005). It might be possible that in specific samples affective symptoms are indeed associated with an increased risk for $A D$, but that apart from the type of $\mathrm{MCl}$, other factors that characterize such samples remain to be identified. Because affective symptoms are common in subjects with $\mathrm{MCl}$, services for subjects with cognitive complaints, should pay attention to this symptomatology, and should have psychiatric expertise available to diagnose and treat these symptoms.

\section{Acknowledgements}

The authors thank Nico Rozendaal for his help in the data management. The study was funded in part by the "Hersenstichting Nederland" (Dutch Brain Foundation). 



\section{Symptoms of preclinical dementia in general practice up to five years before dementia diagnosis}

Ramakers, IHGB., Visser PJ., Aalten, P., Boesten, HMJ., Metsemakers, JFM., Jolles, J. \& Verhey, FRJ.

Published in Dementia and other Geriatric Cognitive Disorders, 24, 300-306. 


\section{Abstract}

Objectives: To investigate which symptoms are indicative of preclinical dementia in general practice and whether subjects with preclinical dementia have an increased contact frequency with their GP.

Methods: Individuals with preclinical dementia $(n=75)$ and non-demented controls $(n=125)$ were selected from the Dutch GP registration network (RNH). Number of visits and odds ratio for the risk of subsequent dementia (OR) of various symptoms were analysed. Analyses were done separately for each 12-month period, in the 5 years prior to the diagnosis of dementia.

Results: In the 5 years prior to diagnosis, subjects with preclinical dementia visited their GP more often than controls. Gait disturbances were the earliest predictor. Cognitive complaints were predictive for dementia in the three years before diagnosis. All other symptoms, except vascular symptoms, were predictive in the year prior to diagnosis. Sensitivity was highest for cognitive symptoms (0.58) and gait disturbances (0.47) in the year before diagnosis.

Conclusion: Preclinical dementia is associated with an increased contact frequency between patient and GP at least five years prior to the diagnosis of dementia. Gait disturbances and cognitive complaints are the earliest symptoms of preclinical dementia.

Keywords: preclinical, dementia, general practice. 


\section{Introduction}

Dementing disorders are often characterized by a preclinical stage in which the individual experiences impairments but is not yet demented. It is important to identify subjects with preclinical dementia so that early interventions can be applied and psychosocial support can be provided.

The general practitioner (GP) plays an important role in the identification of subjects with preclinical dementia given that these subjects or their relatives often contact the GP first with their complaints (Wilkinson et al., 2004). Additionally, GPs are able to detect gradual changes in cognition and behaviour, because they often know their patients for a long time. However, knowledge with respect to which symptoms are suggestive of preclinical dementia in general practice is very limited and this may cause an undesirable delay in time between when the first symptoms present and the diagnosis (Wilkinson et al., 2004). Additionally, early recognition of potential dementing subjects can help GPs to select subjects that may benefit from referral to specialized facilities such as memory clinics for further diagnostics and prevention of disease progression.

The aim of the present study was to investigate how subjects with preclinical dementia present in general practice. We investigated these individuals' contact frequency with their GP in the five years prior to their diagnosis. Additionally, we investigated the symptoms with which these subject presented in those five years. Contact frequency and symptoms of these subjects, were compared with those of control subjects. We hypothesized that subjects with preclinical dementia would have a higher contact frequency with their GP and would also present more complaints that have been previously recognized as early symptoms of dementia or risk factors for dementia in the general population. These symptoms include cognitive impairment, affective symptoms, behavioural problems, vascular problems, gait disturbances, and changes in appetite and weight (Borenstein et al., 2005; Buchman et al., 2005; Copeland et al., 2003; Geerlings et al., 2000; Jonker et al., 2000; Verghese et al., 2002).

\section{Methods}

\section{Subjects}

Subjects were selected from the Registration Network of Family Practices (RNH). The RNH is a computerized and anonymous database that contains patient characteristics and all relevant health problems of subjects from 21 rural and urban GPs in the Southern region of the Netherlands (Metsemakers et al., 1992). The register offers a unique opportunity as a sample frame for research in a general practice setting on which cross-sectional and longitudinal 
analyses can be performed (Metsemakers et al., 1992). When compared with the Dutch population, the RNH population showed a slightly higher mean age. Further, some differences with respect to the marital status were noted (Metsemakers et al., 1992; Van den Akker, 2004).

The cases included in this study consisted of subjects who had a diagnosis of dementia registered in the RNH database between 1996 and 1999. During the study period, GPs in the Netherlands followed the Dutch Guidelines for General Practitioners (NHG) (NHG, 1993) for the diagnosis of dementia, which included the DSM-III-R criteria for dementia (APA, 1987). For each demented subject, two control subjects were randomly selected from the same practice, after matching for age ( \pm five years) and sex. This resulted in 82 demented subjects and 150 control subjects. From this sample we excluded 25 subjects, because insufficient data were available for the period of at least three years prior to diagnosis. We also excluded eight control subjects who had been diagnosed with dementia in the time that passed between the index period and the date of analysis. This was done to prevent contamination of the control group. The final study population consisted of 74 demented patients and 125 non-demented controls (Table 1). The study was approved by the Medical Ethical Committee of the university hospital of Maastricht.

\section{Data collection}

Data were collected on the contact frequency (including GP consultations, telephone consultations and visits) and symptoms presented during each contact. These were collected separately for each 12-month period over the course of the five years prior to the dementia diagnosis. For one control subject and two subjects with preclinical dementia, no data were

Table 1: Baseline characteristics.

\begin{tabular}{lll} 
& $\begin{array}{l}\text { Preclinical dementia group } \\
(\mathrm{n}=74)\end{array}$ & $\begin{array}{l}\text { Control group } \\
(\mathrm{n}=125)\end{array}$ \\
\hline Age, years (SD) & $79(6.2)$ & $79(7.4)$ \\
Sex, \% female & 62 & 61 \\
Marital status, \% married & 53 & 46 \\
Education Low/Middle/High, \% & $81 / 17 / 2$ & $77 / 20 / 3$ \\
Hypertension, \% & 42 & 36 \\
Coronary heart disease, \% & 22 & 24 \\
Stroke, \% & 18 & 11 \\
TIA, \% & 10 & 8
\end{tabular}

$\mathrm{SD}=$ Standard deviation

Educational level: Low = lower general and vocational education; Middle: middle general and vocational education; and High = higher general and vocational education. 
available for the fourth year prior to diagnosis. For the fifth year prior to diagnosis, data was missing for two control subjects and two subjects with preclinical dementia.

All relevant health complaints presented during a contact (in person or by telephone) were entered in the practice file by the GP. We compiled the presenting symptoms at each contact from the anonymous raw data output for the general practice setting and thereby derived 136 different symptoms. We classified these symptoms into six categories, namely: a) cognitive symptoms (including amnesia, forgetfulness, confusion, cognitive decline, orientation problems, language problems, problems with logical thinking, and loss of decorum); b) affective symptoms (including fatigue, irritability, anxiety, sleep-related problems, depressive mood, being upset, loss of initiative, loss of interest, crying, complaining, sadness, hyperventilation, mood changes, and suicidal ideation); c) behavioural symptoms (including restlessness, delusions/hallucinations, aggression/agitation, changes in character, and suspicion); d) vascular symptoms (including chest pains, loss of speech, temporary paralysis, continuous paralysis, loss of strength, decline in vision, and thick tongue); e) gait disturbances (including falls and problems with walking); and f) changes in weight or appetite (including loss of appetite and other weight changes.

\section{Data analyses}

Statistical analyses were performed using the SPSS 11 for Macintosh. The difference in contact frequency between subjects with preclinical dementia and controls were analysed using independent t-tests. The predictive accuracy of the symptoms for dementia was determined using binary logistic regression analyses. The main outcome measure was the odds ratio (OR). Secondary outcome measures were the sensitivity (percentage of subjects with preclinical dementia who expressed the symptom) and the specificity (percentage of control subjects who did not express the symptom). Analyses were done separately for each 12-month period, in the five years prior to dementia diagnosis. All analyses were performed with a two-tailed alpha level of .05 .

\section{Results}

\section{Frequency of contact}

In total, 3837 contacts with the GP were registered during the time period under investigation. In each of the five years, subjects with preclinical dementia showed a significantly higher contact frequency than subjects without preclinical dementia. A notably more pronounced difference between the two groups in the year prior to diagnosis was also demonstrated 
(Figure 1) (five years prior: 3.8 vs. 3.0; four years prior: 4.2 vs. 3.4; three years prior: 4.4 vs. 3.5; two years prior: 4.4 vs. 3.5 ; and one year prior: 6.6 vs. 3.8 ).

\section{Predictors of dementia}

The earliest predictor was complaints about gait disturbances in the fifth year prior to diagnosis ((OR=3.5) Table 2). Gait disturbances were also predictive in the third and final year before diagnosis ( $\mathrm{OR}=3.8$ and 6.1 respectively). Cognitive complaints were associated with the highest risk for dementia, with ORs ranging from 5.3 five years before diagnosis to 56 in the year before the diagnosis. Affective and behavioural symptoms and symptoms related to weight loss and appetite were predictive in the first year prior to diagnosis, with ORs ranging from 3 to 14 . The ORs for all symptoms, except for vascular symptoms, increased progressively over the course of the five years prior to the diagnosis. The ORs of vascular symptoms did not change over time (Table 2). Even after adjusting for age and sex, the significance of the results remained (data not shown).

Specificity was above 0.83 for all symptoms indicating that the control subjects did not frequently report these symptoms (Table 2). Sensitivity for all symptoms increased over the course of the five years prior to diagnosis. The highest sensitivity noted was related to

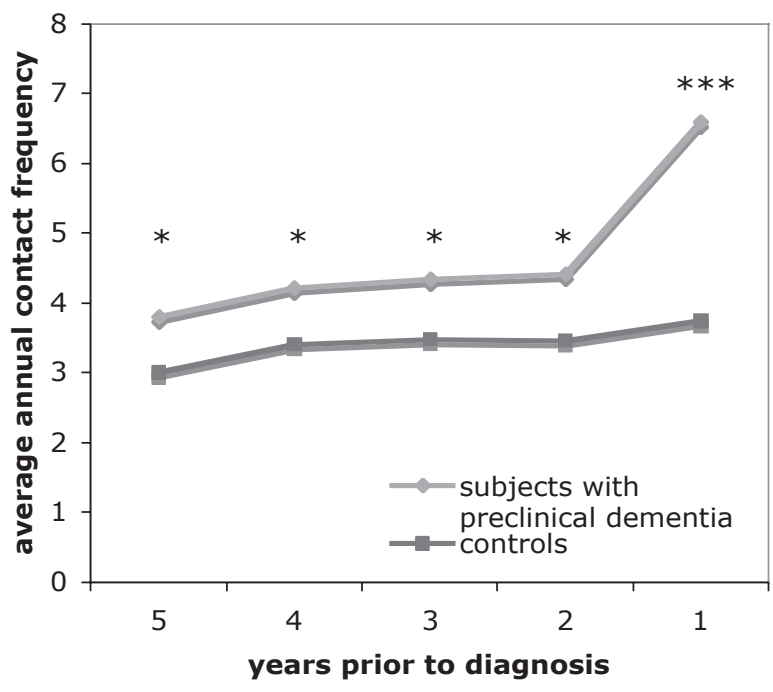

Graph 1: Average number of contacts in the five years prior to diagnosis

${ }^{*} p<.05,{ }^{* * *} p<.001$. 
cognitive symptoms (0.58) and gait disturbances (0.47) in the year before diagnosis was made. This suggests that only a subset of subjects with preclinical dementia report these symptoms to their GP (table 2).

As gait disturbances could also result from a stroke, we performed post-hoc analyses in which we excluded all subjects who have had a stroke in the past $(n=27)$. The predicative accuracy of gait disturbances in subject without a stroke remained essentially the same (first

Table 2: Predictive values of symptoms for the development of dementia.

\begin{tabular}{|c|c|c|c|c|c|}
\hline Symptoms & $\begin{array}{l}\text { Fifth year } \\
\text { prior }\end{array}$ & $\begin{array}{l}\text { Fourth year } \\
\text { prior }\end{array}$ & $\begin{array}{l}\text { Third year } \\
\text { prior }\end{array}$ & $\begin{array}{l}\text { Second year } \\
\text { prior }\end{array}$ & First year prior \\
\hline \multicolumn{6}{|c|}{ Cognitive symptoms } \\
\hline OR $(95 \% \mathrm{Cl})$ & $5.3(0.5-51.8)$ & $2.9(0.9-9.5)$ & $5.4(1.1-28)^{*}$ & $13(3.7-46)^{* * *}$ & $56(16-194)^{* * *}$ \\
\hline Sensitivity & 0.04 & 0.11 & 0.08 & 0.24 & 0.58 \\
\hline Specificity & 0.99 & 0.96 & 0.98 & 0.98 & 0.98 \\
\hline \multicolumn{6}{|c|}{ Affective symptoms } \\
\hline OR $(95 \% \mathrm{Cl})$ & $1.5(0.6-3.7)$ & $0.7(0.3-1.8)$ & $1.0(0.4-2.2)$ & $1.1(0.5-2.3)$ & $3.0(1.5-6.2)^{* *}$ \\
\hline Sensitivity & 0.14 & 0.11 & 0.15 & 0.18 & 0.32 \\
\hline Specificity & 0.9 & 0.85 & 0.85 & 0.83 & 0.86 \\
\hline \multicolumn{6}{|c|}{ Behavioural symptoms } \\
\hline OR $(95 \% \mathrm{Cl})$ & $1.1(0.2-7.0)$ & $0.9(0.2-4.8)$ & $3.5(0.6-20)$ & $2.4(0.8-7.2)$ & $14(3.2-65)^{* *}$ \\
\hline Sensitivity & 0.03 & 0.03 & 0.05 & 0.11 & 0.19 \\
\hline Specificity & 0.98 & 0.97 & 0.98 & 0.95 & 0.98 \\
\hline \multicolumn{6}{|c|}{ Vascular symptoms } \\
\hline OR $(95 \% \mathrm{Cl})$ & $0.7(0.3-1.9)$ & $0.9(0.4-2.1)$ & $1.4(0.6-3.2)$ & $0.96(0.4-2.4)$ & $1.6(0.7-3.5)$ \\
\hline Sensitivity & 0.08 & 0.13 & 0.16 & 0.11 & 0.18 \\
\hline Specificity & 0.89 & 0.86 & 0.88 & 0.89 & 0.88 \\
\hline \multicolumn{6}{|c|}{ Gait disturbances } \\
\hline OR $(95 \% \mathrm{Cl})$ & $3.5(1.2-10)^{*}$ & $1.5(0.6-3.6)$ & $3.8(1.4-11)^{*}$ & $2.2(0.98-4.9)$ & $6.1(3.1-12)^{* * *}$ \\
\hline Sensitivity & 0.15 & 0.14 & 0.16 & 0.2 & 0.47 \\
\hline Specificity & 0.95 & 0.9 & 0.95 & 0.9 & 0.87 \\
\hline \multicolumn{6}{|c|}{$\begin{array}{l}\text { Changes in weight } \\
\text { and appetite }\end{array}$} \\
\hline OR $(95 \% \mathrm{Cl})$ & $0.6(0.1-2.8)$ & $0.8(0.2-3.5)$ & $1.1(0.4-3.0)$ & $1.8(0.5-5.6)$ & $5.9(2.2-16)^{* * *}$ \\
\hline Sensitivity & 0.03 & 0.04 & 0.09 & 0.08 & 0.23 \\
\hline Specificity & 0.95 & 0.95 & 0.91 & 0.95 & 0.95 \\
\hline
\end{tabular}


year prior: $\mathrm{OR}=7.3, \mathrm{p}<.001$; second year prior: $\mathrm{OR}=2.5, \mathrm{p}<.05$; in the third year prior: $\mathrm{OR}=$ $5.2, p<.01$; fourth year prior: $O R=1.3, p>.05$; and fifth year prior: $O R=3.3, p<.05$ ).

\section{Discussion}

Preclinical dementia is associated with increased contact frequency to the GP at least five years prior to the diagnosis of dementia. Symptoms that could best predict dementia in the general practice setting were cognitive symptoms and gait disturbances.

The observation that subjects with preclinical dementia had an increased contact frequency up to five years before the dementia diagnosis supports and extends the findings of a previous study in which an increased contact frequency two years before the diagnosis of dementia was found (Albert et al., 2002). It is possible that the increase in contact frequency occurred because patients experienced symptoms related to the dementing disorder. Alternatively, subjects with preclinical dementia may have had more often control visits for conditions that are risk factors for dementia, such as hypertension or diabetes. However, a post-hoc analysis indicated that control visits were equally common in both groups (data not shown). The increase in contact frequency for subjects with preclinical dementia in the year prior to diagnosis may have been the result of the patient entering the diagnostic phase in which the dementia was established.

The earliest predictor of dementia was gait disturbances. This was already predictive in the fifth year prior to diagnosis. It is also important to note that cognitive complaints were associated with the highest risk for dementia. For most predictors included in this study, the OR increased over the course of five years from to diagnosis. As expected, the predictors were most notable in the final year before diagnosis. The high ORs for the symptoms in the final year may be partly explained by circularity since the presence of some of the symptoms likely initiated the process by which the diagnosis of dementia could be made.

Complaints about everyday memory problems have been associated with cognitive impairment and dementia in several clinical and population-based studies (Jonker et al., 2000). Although cognitive symptoms are a key feature of dementing disorders, in our study, cognitive complaints are indicative of preclinical dementia only in the final two years prior to diagnosis. In all other years, a low predictive accuracy was found. Further, cognitive symptoms were not expressed in the entire sample but only in a subgroup of subjects with preclinical dementia and ranged from $4 \%$ five years prior to diagnosis to $58 \%$ in the year before diagnosis. $A$ possible explanation for this finding is that the many of the subjects with preclinical dementia considered cognitive impairments normal for their age. Alternatively, they may have been ashamed of their impairments and thus choose to not reported them. It is also possible that, 
as a result of the dementing process, subjects were unaware of cognitive changes (Tabert et al., 2002). A final option was that, especially in the fourth and fifth years prior to diagnosis, preclinical subjects simply did not experience any cognitive impairment. Our findings with respect to the low predictive accuracy of cognitive impairments for the group with preclinical dementia corresponds with previous studies in which many subjects with preclinical dementia did not express cognitive complaints (Ganguli et al., 2006; Palmer et al., 2003; Tabert et al., 2002). However, because our findings are based on contact notes of the GP and because it is possible that the GPs did not take sufficiently detailed notes during their contacts with patients, we may have underestimated the frequency of cognitive symptoms.

Our finding that gait disturbances predict dementia is in agreement with recent research that suggests that gait disturbances in aging may be indicative for a cognitive impairment that can be objectified several years later (Scherder et al., 2007; Verghese et al., 2002). A population-based study demonstrated that subjects with gait abnormalities had an increased risk of developing dementia, especially non-Alzheimer's dementia (Verghese et al., 2002). In addition, another study noted that fall events occurred in $42 \%$ of the participating mild to moderate dementia subjects (Horikawa et al., 2005). The present study indicated that gait disturbances were reported long before the diagnosis was made and that these disturbances were even quite frequent even five years prior to the diagnosis. In this fifth year prior to diagnosis, gait disturbances (15\%) were much more frequent than cognitive symptoms (4\%). This finding is similar to the study of Ganguli et al. (2006) who, using primary care charts, established that falls occurred in $15 \%$ of subjects with a CDR total box score of 0.5 , compared to memory complaints in only $8 \%$ of subjects (Ganguli et al., 2006). The specificity of gait disturbances for dementia in our study was high (above .87). Whether this high specificity translates to a high positive predictive value depends on the incidence of dementia, which could not be investigated in this study because of the case-control design.

The finding that gait disturbances are a sign of early dementia is in line with recent research, summarized by Scherder et al. (2007). They concluded that there is a strong relationship between gait and cognition (Scherder et al., 2007), gait and gait-related motor disturbances being present in all subtypes of dementia, even in the early and preclinical stages. Neural support for this relationship was based on recent studies that found a strong involvement of the hippocampus in gait, because of his connections with the prefrontal cortex and the striatum (Scherder et al., 2007).

A decline in gait and balance is not only associated with vascular white matter lesions (Whitman et al., 2001), but also in Alzheimer's disease, which is consistent with our post-hoc analyses, in which we showed that after exclusion of subjects with stroke, gait disturbances were 
still predictive of dementia. Waite et al. (2005) found that subjects with cognitive impairment in combination with impaired motor control were at higher risk for developing dementia than subjects with cognitive impairment alone (Waite et al., 2005).

Affective symptoms were a marker of preclinical dementia only in the year prior to diagnosis. In the other years, affective symptoms were equally common among subjects with preclinical dementia and the control subjects. This finding does not correspond with a recent meta-analysis in which the conclusion presented was that a history of depression may be an independent risk factor for Alzheimer's Disease (AD) (Ownby et al., 2006). Our findings also fail to support another conducted study in a general practice setting in which a significant relationship between old-age depression and subsequent dementia was found (Buntinx et al., 1996). Had we conducted a depression diagnosis, rather than merely documenting affective symptoms we may have possibly been able to demonstrate greater predictive power of affective symptoms and, more especially depression. Another possible explanation for this lack of predictive power was that the present study included only subjects who visited the GP. It is possible that affective symptoms have more discriminative power in the general population.

Behavioural symptoms and changes in weight and appetite were only predictive in the year preceding diagnosis. This suggests that these symptoms develop later in the neurodegenerative process or, alternatively, that these symptoms only became severe enough to report to the GP in the final year prior to diagnosis.

In contrast to our expectations, the frequency of contact with the GP as a result of vascular symptoms did not differ significantly between subjects who developed dementia and subjects who did not. The prevalence in both groups was approximately $15 \%$. A possible explanation for this is that the vascular complaints that patients presented to their GP in this study may not have been specific for underlying vascular pathology. When we excluded symptoms that were thought not to be related to a vascular disorder, results were essentially the same.

In the RNH database dementia is used as diagnostic entity rather than dementia subtypes. Still, because the RNH database registered all relevant health care problems an indicator for vascular causes of the dementia could be inferred. Of the 74 patients with dementia, 13 subjects (18\%) had experienced a stroke before the diagnosis of dementia and 31 subjects (42\%) of the subject were known with hypertension. Thus dementia might have had resulted from stroke in about $20 \%$ of the subjects, while small vessel disease might have contributed to the dementia syndrome in about $40 \%$.

Dementia was diagnosed according to DSM-IIIR criteria (APA, 1987). The GP, however, might have missed the diagnosis at earlier visits, because demented subjects may have been unaware of their cognitive impairment, especially when an informant was absent. Therefore, in 
some subjects symptoms considered to reflect preclinical dementia might have been symptoms of dementia. Cognitive tests might have been useful to identify subjects with dementia in an earlier stage, but such tests were not used for screening purposes on a routine basis, but only when cognitive impairment was suspected.

One of the strengths of our study was the large size of our study population. A second strength was our use of a matched control design in which subjects were included from both rural and urban general practices. In doing this, we were able to increase the generalizability of our findings. Our inclusion of five years worth of data is an additional strength. Lastly, by following up on control subjects after the index date, we were able to exclude control subjects that had developed dementia in the period between the index date and date of analyses, and thereby avoided contamination in so far as was possible.

Our study also had several limitations. Our reliance on reports written by GPs can be considered a limitation. Despite continuous note-taking training (Buntinx, 1994), it is possible that some of the reports were incomplete. Additionally, we were not able to obtain data on the type of dementia the preclinical subjects developed. An additional limitation worthy of attention is the broad Cls of some of the predictors that were noted during the regression analyses. It is possible that these broad $\mathrm{Cl}$ s were caused by the relative infrequency of several of the reported complaints. The classification of some symptoms might be arbitrary as some symptoms could be classified in more than one category, especially for symptoms classified as cognitive, affective, or behavioural symptoms. When loss of decorum (classified into the cognitive category) and irritability (classified into the affective category) were classified as behavioural symptoms, however, the results did not change. Finally, our findings may not applicable in different settings and in younger subjects.

The GP plays a crucial role in the timely recognition and early identification of subjects at risk for dementia (Artero \& Ritchie, 2003; Barrett et al., 1996; Wilkinson et al., 2004). The present study showed that complaints of cognitive performance and gait should alert the GP for incipient dementia. If these symptoms are expressed, the GP may do a formal assessment of cognition, refer the patient for further diagnostic evaluation, or keep the patient under clinical supervision. Given that almost half of the subjects did not actively report cognitive symptoms in the year prior to the diagnosis, we contend that the GP must play an active role in identifying subjects at risk for dementia by bringing together all the available information related to the medical, cognitive, and daily functioning of their patients. By referring to symptoms that present during consultations, GPs are not only in a position whereby they can identify subjects at risk for developing dementia, they can also start a process by which additional diagnostic testing can be conducted, and follow these subjects over time. 



$$
\text { Part } 2
$$





\section{Determinants of help-seeking behaviour}

in subjects with subjective memory complaints at a memory clinic: a casecontrol study

Ramakers, IHGB., Visser, PJ., Bittermann, AJN., Ponds, RWHM., van Boxtel, MPJ., Verhey, FRJ. 


\section{Abstract}

Objective: Memory complaints in the absence of objective test impairments are common. Only a subset of these subjects seeks medical attention for these complaints. The aim of the present study was to investigate which factors determine why people with subjective memory complaints (SMC) seek medical attention.

Methods: Thirty-three cases with SMC from a memory clinic were compared to 85 control subjects with SMC from a population-based study who did not seek help for their complaints. We investigated whether cases differed from controls with respect to the following: depressive and anxiety symptoms (SCL-90), extraversion and neuroticism (EPQ), meta-memory (MIA), quality of life (SF-36), changes in memory and daily functioning according to a relative (DECO), life-changing events, and a family history of dementia.

Results: Cases with SMC who seek medical attention, scored lower on memory self-efficacy and quality of life. They were more often worried due to a positive family history of dementia by comparison to the control subjects. Relatives of cases reported more deterioration in daily functioning than relatives of controls. Both the cases and control subjects had similar levels of depressive and anxiety symptoms, as well as levels of extraversion and neuroticism.

Conclusion: Lower memory self-efficacy and quality of life, deterioration in daily functioning, and worries due to a positive family history for dementia are factors that determine why subjects with SMC seek medical attention. This information may be useful for the development of interventions for these subjects.

Key words: memory complaints without impairment, subjective memory complaints, memory self-efficacy, affective symptoms, memory clinic. 


\section{Introduction}

Memory complaints are very common in middle-aged and older subjects (Jonker et al., 2000; Ponds et al., 1997), and occur in about $40-50 \%$ of the subjects older than 55 years (Ponds et al., 1997). Formal cognitive testing reveals that about half of these subjects do not have objective memory impairments (Gallassi et al., 2008). Hence, they are considered to have subjective memory complaints (SMC). Only a subset of subjects with SMC decides to seek medical attention for these complaints, and about $15-20 \%$ of the subjects seen at memory clinics actually do have SMC (Verhey et al., 2007). The reason why subjects with SMC seek medical attention remains largely unknown. One study found that cognitive, psychiatric and health related factors played a role in the decision to visit a doctor for cognitive complaints, but this study did not focus on SMC only and also included complainers with objective impairments (Jorm et al., 2004). Understanding the reasons why subjects with SMC decide to seek medical attention is important to determine the needs of these subjects and to provide adequate treatment and advice.

SMC can reflect normal aging or self-awareness of underlying degenerative pathology (Barnes et al., 2006; Schmand et al., 1996). However, it may also be related to other factors, such as psychiatric symptoms, personality characteristics and poor physical health (Cargin et al., 2007; Comijs et al., 2002; Derouesne et al., 1999; Jorm et al., 2004; Ponds et al., 1997).

The aim of the present study was to investigate which factors determine the decision to seek help for SMC. We compared cases with memory complaints, but lacking objective memory impairments, who did decide to visit a memory clinic to healthy subjects with memory complaints who did not seek medical attention.

We hypothesized that non-cognitive symptoms such as depression, anxiety, and personality factors such as neuroticism and extraversion, would be more frequent in cases with SMC who visit a memory clinic than in subjects with SMC who do not seek medical care (Cargin et al., 2007; Comijs et al., 2002; Derouesne et al., 1999; Jorm et al., 2004; Ponds et al., 1997). We also hypothesized that, compared to subjects who do not visit a memory clinic, the cases who visit a memory clinic had a lower quality of life (Mol et al., 2007), had less knowledge about their own memory functioning (Ponds \& Jolles, 1996a), were more often worried about dementia (Commissaris et al., 1994) due to a family history of dementia, had more often experienced a life-changing event in the past year, and had experienced more changes in memory and daily functioning, as reported by a caregiver (Streams et al., 2003). 


\section{Methods}

\section{Cases from the Maastricht Memory Clinic}

Subjects who did seek medical attention for their SMC were selected from an ongoing cohort study at the Maastricht Memory Clinic (MMC). The MMC is an outpatient clinic of the university hospital Maastricht (Verhey et al., 1993). For the present study, we selected consecutive patients (referred to as 'cases') at the time of their first visit to the MMC if they fulfilled the following criteria: they were 50 years or older, had SMC but lacked objective memory impairment, which was defined as a score below -1.5 SD on the delayed recall test of the Auditory Verbal Learning Test (AVLT) (Van der Elst et al., 2005), had a score of 2 or 3 on the Global Deterioration Scale (GDS) (Reisberg et al., 1982), were not demented, and at baseline they had no identified cause for their cognitive impairment, such as cerebrovascular disorders, brain trauma, endocrine disorders or severe psychiatric disorders (Visser et al., 2000).

Eligible cases were mailed information about the study, questionnaires, a written informed-consent form, and a self-addressed return envelope in which to return the questionnaires and the signed informed consent. Fifty-two cases were eligible for inclusion. Subjects who did not return the questionnaires were contacted by telephone. Eighteen cases refused to participate and 1 subject returned an incomplete questionnaire. Thus 33 cases (63\%) were included. Cases who did not participate in the study $(n=19)$ had a lower level of education and were about 8 years older than the cases who did decide to participate. Sex distribution and mean MMSE score did not differ between the groups.

\section{Subjects from the Maastricht Aging Study (MAAS):}

The eligible cases selected from the memory clinic were compared to subjects from the Maastricht Aging Study (MAAS) (referred to as 'controls'), a longitudinal population based study into the determinants of cognitive aging (Jolles et al., 1995). Participants in the MAAS were recruited from the Registration Network Family Practices (RNH) (Metsemakers et al., 1992) composed by 42 general practitioners in the province of Limburg, the Netherlands. Subjects with medical conditions that could interfere with cognition were identified using the RNH records and excluded from the sample frame. For the present study, we selected subjects from this sample if: they were 50 years or older, had memory complaints as measured with the question 'Do you find yourself forgetful?', but lacked objective memory impairment (below -1.5 SD) according to the delayed recall test of the AVLT, and had not sought professional help for their memory complaints. In total, 83 controls were eligible for comparison with the MMC patients. The Medical Ethical Commission of the Academic Hospital Maastricht approved this 
study. After the study was explained to them, both controls from MAAS and cases from the MMC gave their written informed consent.

\section{Instruments}

Memory self-efficacy (MSE). The abridged Dutch Metamemory in Adulthood questionnaire (MIA) (Dixon et al., 1988) was used to measure MSE. MSE consists of the MIA subscales capacity, change and anxiety, and reflects beliefs about the personal competence in various memory situations. Subjects rate statements about themselves on a five point Likert scale, ranging from 'strongly disagree' to 'strongly agree'. Higher MIA MSE scores indicate higher self-efficacy ratings for memory. Specifically, the subscale capacity consists of 12 items, and a higher score on this subscale indicates greater perceived memory capacity. The change subscale consists of 10 items and a higher score indicates less perceived decline (or more stability) in memory functioning. The subscale anxiety consists of 12 items, in which a higher score indicates more feelings of anxiety and/or distress about one's memory functioning.

Mood symptoms. The subscales depression (16 items) and anxiety (10 items) of the Dutch Symptom Check List (SCL-90) (Arrindell \& Ettema, 2003; Derogatis \& Cleary, 1977) were used to measure depressive and anxiety symptomatology. The SCL-90 is a multidimensional selfrating questionnaire (Derogatis \& Cleary, 1977), and is a widely used standardized inventory of psychiatric symptomatology. Questions are rated on a five point Likert scale, ranging from "strongly disagree" to "strongly agree". A higher score on these subscales indicate more symptoms of depression or anxiety.

Personality symptoms. A shortened version of the Eysenck Personality Questionnaire (EPQ-BV) was used to measure symptoms of extraversion and neuroticism. This shortened version of the EPQ (Sato, 2005) contained 24 items. It is a self-reporting questionnaire based on a twopoint scale with the response options "yes" and "no". The EPQ-BV had coefficient alphas for the extraversion and neuroticism scales of .92 and .90 respectively (Sato, 2005). Furthermore the test-retest reliability values of the two scales turned out to be .92 for both the extraversion and neuroticism measures.

Quality of life. The physical and mental summary scores of the Short Form Health Survey (SF36) (Van de Zee et al., 1993) were used to measure quality of life related to physical and mental aspects. The SF-36 is composed of 36 items measuring health across eight dimensions: physical functioning, social functioning, role limitations due to physical problems, role limitations due to emotional problems, pain, mental health, vitality, and general health perception. These eight subscales can be summarized into one physical and one mental component summary score. Each dimension score ranges from 0 to 100. Higher scores indicate better health-related quality of life. 
Changes in memory functioning. The Détérioration Cognitive Observée (DECO) (Ritchie \& Fuhrer, 1992) was used to measure changes in memory and daily functioning in the past year. The questionnaire is completed by a close relative who had at least monthly contact with the patient. The DECO consists of 19 items, scored on a 3-point Likert scale consisting of "the same or better", "less good" and "significantly less good". A higher score on the DECO indicates better memory and daily functioning of a subject in experience of the relative.

Other variables. The Mini Mental State Examination (MMSE) (Folstein et al., 1975) was used to measure the general cognitive status. Level of education (LE) was defined by classifying formal schooling in a system often used in the Netherlands, which is comparable to the International Standard Classification of Education (UNESCO, 1976). The participants were grouped as follows: those with at most primary education (low), those with junior vocational training (average) and those with senior vocational or academic training (high) (Van der Elst et al., 2005). Additionally, we asked the subjects (a) about their fear for developing dementia, including the reason, and (b) whether they had experienced important life-changing events during the past year. Objective memory performance was investigated with the AVLT (Van der Elst et al., 2005).

\section{Statistics}

All data were processed with Statistical Package for Social Sciences (SPSS) version 11.0.4 for Mac OS X. Group comparisons for variables at interval or ratio level were performed, using independent t-tests or Mann-Whitney $U$ tests. Dichotomous variables were analyzed using Chi-square tests. All analyses were performed at a two-tailed alpha level of .05. Based on the main outcome measure (the MIA-MSE), by 33 cases and 83 control subjects, and a two-tailed testing, a power of 0.69 was reached. Based on our hypothesis that cases had lower MIA-MSE scores compared to controls, a one-tailed analysis resulted in a power of 0.79 .

Table 1: Baseline characteristics.

$\begin{array}{llll} & \begin{array}{l}\text { Cases } \\ (\mathrm{n}=33)\end{array} & \begin{array}{l}\text { Controls } \\ (\mathrm{n}=83)\end{array} & \mathrm{p} \\ \text { Age, years }( \pm \mathrm{SD}) & 62.0(8.6) & 65.1(10.3) & \mathrm{ns} \\ \text { Sex }(\% \text { male }) & 61 & 47 & \mathrm{~ns} \\ \text { LE }(\% \mathrm{~L} / \mathrm{M} / \mathrm{H}) & 27 / 33 / 40 & 49 / 36 / 15 & .008 \\ \text { MMSE score }( \pm S D) & 28.8(1.5) & \mathrm{ns} \\ \text { Delayed recall, Z-score }( \pm \mathrm{SD}) & 0.0(1.0) & 0.3(1.1) & \mathrm{ns}\end{array}$

$\mathrm{Ns}=$ not significant at $\mathrm{p}$-value $.05, \mathrm{SD}=$ Standard deviation, $\mathrm{LE}=$ level of education: $\mathrm{L}=$ low, $\mathrm{M}=$ medium, $\mathrm{H}=$ high. 


\section{Results}

\section{Subject characteristics}

Baseline characteristics of cases and controls are shown in Table 1. There was no difference in age, sex, performance on the immediate recall test, or general cognitive status as measured with the MMSE between the two groups. Cases were on average higher educated by comparison to control subjects. Because of this difference we repeated all analyses with correction for education. These analyses yielded similar findings as without correction. Presented data in the present study are without correction for education.

The average scores on the questionnaires by the cases and control subjects are presented in Table 2. Cases had significantly lower MSE scores on the MIA than the controls. More detailed analyses of the MIA-MSE score showed that cases had a significantly lower score on the subscale capacity, as well as a significantly lower score on the subscale change indicating less stability in memory performance. Cases scored significantly higher on the DECO compared to the control subjects. Cases had a significant lower mental summary score on the SF-36 (Table 2), whereas the physical summary score was equal in both groups.

The proportion of subjects who reported to be worried about developing dementia was similar in both groups. The proportion of subjects who reported to be worried about

Table 2: Differences on rating scales between cases and controls.

$\begin{array}{llll} & \text { Cases } & \text { Controls } & \mathrm{p} \\ & \mathrm{n}=33 & \mathrm{n}=83 & \mathrm{~ns} \\ \text { SCL-depression } & 39.5 \pm 14.5 & 35.0 \pm 10.6 & \mathrm{~ns} \\ \text { SCL-anxiety } & 24.3 \pm 8.7 & 21.2 \pm 7.8 & \mathrm{~ns} \\ \text { EPQ-extraversion } & 5.2 \pm 2.9 & 5.7 \pm 3.0 & \mathrm{~ns} \\ \text { EPQ-neuroticism } & 5.3 \pm 3.3 & 4.8 \pm 3.5 & 0.009 \\ \text { MIA-MSE } & 83.6 \pm 17.8 & 91.3 \pm 12.4 & 0.037 \\ \text { DECO-total } & 31.0 \pm 6.4 & 33.7 \pm 4.2 & \mathrm{~ns} \\ \text { SF-physical component score } & 44.8 \pm 10.9 & 45.6 \pm 9.5 & 0.010 \\ \text { SF-mental component score } & 42.2 \pm 13.6 & 49.3 \pm 8.8 & \mathrm{~ns} \\ \text { Worried to develop dementia (\% yes) } & 70 & 57 & 0.048 \\ \text { Worried because of family history of dementia (\% } & 60 & 32 & \mathrm{~ns} \\ \text { yes) } & & 66 & \\ \text { Life-changing event (\% yes) } & 67 & & \end{array}$

Data are presented as mean \pm standard deviation, $\mathrm{ns}=$ not significant, $\mathrm{SCL}=$ Symptom Check List, $\mathrm{EPQ}=$ Eysenck Personality Questionnaire, MIA = Metamemory in Adulthood, MSE = Memory Self-Efficacy, DECO $=$ Détérioration Cognitive Observée, SF = Short Form Health Survey-36. 
dementia because they had a positive family history of dementia was significantly higher in cases than in control subjects (Table 2).

Cases and control subjects had comparable scores on the subscales depression and anxiety of the SCL-90. The scores on both subscales were 'high' to 'very high', compared to the SCL-90 standards for the normal population (Arrindell \& Ettema, 2003). No significant difference was found for the EPQ-BV subscales extraversion and neuroticism between cases and controls. The proportion of subjects that had experienced a life-changing event in the past year was also comparable in the two groups.

\section{Discussion}

The main finding of this study is that cases who sought help for their SMC at a memory clinic were characterized by less personal competence in memory related situations and a lower quality of life related to mental health by comparison to controls. In addition, cases showed more decline in memory functioning as reported by a relative, they were also more often worried about dementia due to a positive family history for dementia, and had more often a higher level of education. Symptoms of depression, anxiety, extraversion and neuroticism did not differ between cases and controls.

Cases had more negative beliefs about their personal competence in various memory situations, reflected by lower MIA-MSE scores. By comparison to control subjects, cases reported less memory capacity and more decline (or less stability) in memory functioning despite the fact that they had normal cognitive functioning according to the MMSE and a memory test. This suggests that the personal (subjective) evaluation of one's own memory functioning is an important factor in determining help-seeking behaviour.

Our study showed that cases who had sought medical attention, reported a lower quality of life related to mental health by comparison to controls who had not sought help. The quality of life related to physical health was equivalent for cases and controls. The existence of a relation between memory complaints and a diminished quality of life in the elderly was also indicated by a recent review (Mol et al., 2007). However, a study by Jorm et al. (2004) reported that both the subjects who did visit a doctor or their memory complaints and subjects who did not visit a doctor had comparable mental summary scores. In contrast, this study found a significant difference between these groups with regard to the physical summary score. The differences in findings between the study by Jorm et al. (2004) and the present one can possibly be explained by differences in setting, by the use of another version of the SF for investigating quality of life, and by the specialty of the doctor who is visited for the memory complaints. 
Relatives of cases more frequently reported a decline in memory functioning and in functioning during daily activities in the past year than the relatives of control subjects. This is in line with the findings of Streams et al. (2003), who found that changes in cognitive or non-cognitive aspects reported by the caregiver accounted for $81 \%$ of the trigger events to seek help at a memory clinic. Cases also more often reported a positive family history of dementia as cause for their worries about their forgetfulness. Therefore, cases with a positive family history of dementia may be more prone to interpret their cognitive complaints in a more catastrophic way. Such a negative cognition may play an important role in the development and maintenance of the memory complaints.

Both groups studied here reported high levels of depressive and anxious symptomatology compared to normative data of the SCL for the healthy population (Arrindell \& Ettema, 2003). This is in line with other studies reporting higher depression and anxiety scores in subjects with memory complaints (Cargin et al., 2007; Jorm et al., 2004; Jungwirth et al., 2004; Lautenschlager et al., 2005; Ponds \& Jolles, 1996b). As cases and controls did not differ in the levels of depressive and anxiety symptoms, these symptoms appear not to be determinants for visiting a memory clinic. The same was true for the personality factors extraversion and neuroticism. This is in line with a study of Jorm et al. (2004), which found similar levels of extraversion and neuroticism between subjects who visit a doctor for their cognitive complaints and subjects who did not. The average levels of extraversion and neuroticism are comparable to those in other studies about subjects with memory complaints using the EPQ (Jorm et al., 2004). Thus, psychopathological and personality symptoms are present in subjects with memory complaints, but they do not appear to be determinants of seeking help at a memory clinic.

Both groups studied here also have comparable MMSE and memory delayed recall scores. Moreover, the scores of both groups were also within normal limits, indicating that the severity of cognitive impairment, or more especially memory impairment was no explanation for deciding to visit a memory clinic in this study.

A recent inventory about the organization and functioning of memory clinics in the Netherlands showed that about $15 \%$ of the memory clinic visitors did not have a cognitive impairment (Verhey et al., 2007). The findings of the present study are important to develop interventions with regard to memory-related cognitive mechanisms for this group of memory clinic visitors. These interventions should focus on improving the subject's knowledge about their own memory functioning, and changing negative beliefs and expectations about their memory. Pharmacological therapy was routine in $97 \%$ of the Dutch memory clinics, while cognitive therapy was part of the standard treatment options in only $24 \%$ of the memory clinics (Verhey et al., 2007). Our findings imply that besides pharmaceutical therapies for 
improving cognitive functioning and psychopathological symptoms, memory clinics should offer cognitive-behavioural therapies to those patients with memory complaints who lack objective impairments according to testing. The therapies would include instructions on how to cope with the memory complaints and on how to improve subjective memory functioning and quality of life related to mental health.

However, subjects with memory complaints should be invited for follow-up assessments to verify the course of the subjective complaints and the objective test performances, because a progressive course of cognitive dysfunctioning caused by underlying brain degeneration cannot be excluded in a cross-sectional assessment. Indeed, preliminary data showed that of the 27 cases with follow-up data for at least one year, three went on to develop dementia compared to none of the subjects from the control group.

To our knowledge, this is the first study in which a comparison is made between clinical subjects with memory complaints not classified as objective impairments who visit a memory clinic for their memory complaints on the one hand, and subjects from the general population who did not show this help-seeking behaviour on the other. Both groups were recruited from the same geographical region and both used for ageing research. Several non-cognitive aspects were taken into account. Cases were selected from a memory clinic setting and findings may not be applicable to other settings. The older age and the lower educational level of the cases who did not participate in the study $(n=19)$ could also have biased our results. Another point of attention is the way in which memory complaints were assessed in either group. Cases were referred to the memory clinic because of memory complaints, whereas control subjects were asked if they find themselves forgetful. A recent study found, however, that whether a single question to identify memory complaints or a more detailed questionnaire is used, did not have an influence on the ability to differentiate between individuals with memory deficits or normal subjects (Cargin et al., 2007). The comparable MMSE and memory scores in both groups also imply that the way memory complaints were investigated did not influence the severity of the cognitive impairments in either group.

\section{Conclusion}

Compared to people with SMC who do not seek help, subjects with SMC visiting a memory clinic are characterized by less subjective memory self-efficacy, a decreased quality of life related to mental health, more deterioration in memory and daily functioning reported by an informant, and more worries related to a family history of dementia. These findings may help to develop adequate interventions for subjects without objective memory impairments who visit a memory clinic due to memory complaints. 


\section{Memory clinics in the Netherlands: also a service of non-demented subjects with cognitive complaints?}

Verhey, FRJ., Ramakers, IHGB., Jolles J., Scheltens, Ph., Vernooij-Dassen, M. \& Olde Rikkert, $M$. 


\section{Abstract}

Background. Memory Clinics (MC's) are multidisciplinary teams involved with early diagnosis and treatment of people with dementia. The aim of this study was to investigate whether, and how these facilities can play a role in not-demented subjects, such as subjects with Mild Cognitive Impairment (MCI).

Methods. A questionnaire with questions about the organization of the $\mathrm{MC}$, the distribution of diagnoses, diagnostics, the aetiology of $\mathrm{MCl}$, used $\mathrm{MCl}$-criteria, and treatment was sent to all MC's in the Netherlands.

Results. The number of MC's increased from 12 to 40 . The number of referrals per service has also increased. The focus is less exclusively on academic centres. Dementia was the most important syndromal diagnosis (62\%), but also $\mathrm{MCl}(23 \%)$ and no cognitive disorder (15\%) were common. However, there was a large variability between the settings in the proportion of the diagnoses dementia/MCl/no cognitive disorder. $92 \%$ of the settings used the concept $\mathrm{MCl}$. The most common diagnostic classifications of subjects with $\mathrm{MCl}$ were primary degenerative, vascular and not specified. In $84 \%$ of the settings the treatment of $\mathrm{MCl}$ subjects was different to that of demented patients, which mainly implicated a neuropsychological follow-up assessment.

Conclusion. MC's are part of standard care for people with early dementia, but MC's play also an increasing role in the diagnostic and therapeutic procedures of subjects with milder cognitive problems. Because of the large variability between the settings in the proportion of the diagnoses dementia/ $\mathrm{MCl} / \mathrm{no}$ cognitive disorder, the treatment possibilities should be fitted to the main problems seen at a particular MC.

Key words. Memory clinic, Alzheimer's disease, Mild Cognitive Impairment, and diagnostics. 


\section{Introduction}

Memory Clinics ( $M C^{\prime}$ 's) are a relative new phenomenon in health care. The first MC's were set up in North America in the mid 1970s, and little later in the UK (Wright \& Lindesay, 1995). In 1986 the first MC developed in the Netherlands in Maastricht and Tilburg (Verhey et al., 1987). Nowadays, MC's are an important service in health care. MC's developed in various ways. They had a large variation in their organization, working methods and services, but shared primary principles of being multidisciplinary teams, involved with the early diagnosis and treatment of people with dementia (Lindesay et al., 2002; Passmore \& Craig, 2004). Although MC's aimed the early diagnoses of dementia, the name 'memory clinic' suggests that this facility also deliver service for non-demented subjects with milder memory problems, such as Mild Cognitive Impairment $(\mathrm{MCl})$. $\mathrm{MCl}$ is a condition that refers to cognitive impairment in subjects without dementia. $\mathrm{MCl}$ is a heterogeneous concept and can have many causes. Therefore the usefulness of this concept in clinical practice is still under debate (Whitehouse \& Moody, 2006). Nevertheless, there is abundant evidence that subjects with $\mathrm{MCl}$ are at increased risk for developing dementia (Geslani et al., 2005; Petersen et al., 1999; Visser et al., 2006). However, not all subjects with $\mathrm{MCl}$ develop dementia in future (Visser et al., 2000), as the cognitive functioning of these subjects can also remain stable or improve over time. In these subjects cognitive impairments can be caused by other somatic, mood, or psychological factors (Jorm et al., 2004; Ponds \& Jolles, 1996a). The differentiation between normal and pathological aging is important, because subjects could benefit from other sorts of treatment, and also because subjects often worries about their memory complaints and want a decisive answer and a prognosis.

In 1998, an overview was published about the organization and working methods of the MC's in the Netherlands that were operational at that time (Verhey et al., 1999). The main findings of that study were that MC's were mainly associated with university hospitals, and were involved in scientific research, mainly clinical trials. Since 1998, several new MC's has arisen. In order to attain more insight into the development and functioning of MC's we compared the data of two inventories, one of 1998 and the other of 2004. Specifically, we focused on the function of MC's in non-demented subjects such as subjects with $\mathrm{MCl}$ and subjects without cognitive disorder.

\section{Methods}

We sent a questionnaire and a self-addressed return envelope to the coordinators of all MC's in the Netherlands. Settings that did not respond, were attended again by telephone or by 
mail. Forty-eighty settings were addressed. Forty settings identify themselves as a MC, from these 37 settings returned the questionnaire (response 93\%). Reasons mentioned for not being a MC were not working in a multidisciplinary way, no medical diagnostics, and being in the starting phase. A list of identified MC's is available at www.alzheimercentrumlimburg. $\mathrm{nl}$. The questionnaire pertained to the organization of the $M C$, the distribution of diagnoses dementia/MCl/no cognitive disorder, diagnostics, the aetiology of $\mathrm{MCl}$, used $\mathrm{MCl}$-criteria, and treatment. $\mathrm{MCl}$ was in this case not restricted to the amnestic criteria, but instead we used a broad definition of all subjects who reported an impairment in any cognitive domain. Statistical analyses were performed using the Statistical Package for Social Sciences (SPSS), version 11 for Mac OS X. Group differences between the coordinating disciplines were tested using ANOVA's or Chi-square tests.

\section{Results}

In 2004, 40 MC's were identified. All MC's were associated with a general or academic hospital. Data about the years of starting up was known from 35 settings and is shown in Figure 1. There is a large increase in number of MC's since 1998, before which data only 12 MC's were operational (Figure 2). In 2004, the MC's were more equally distributed over the country. The disciplines

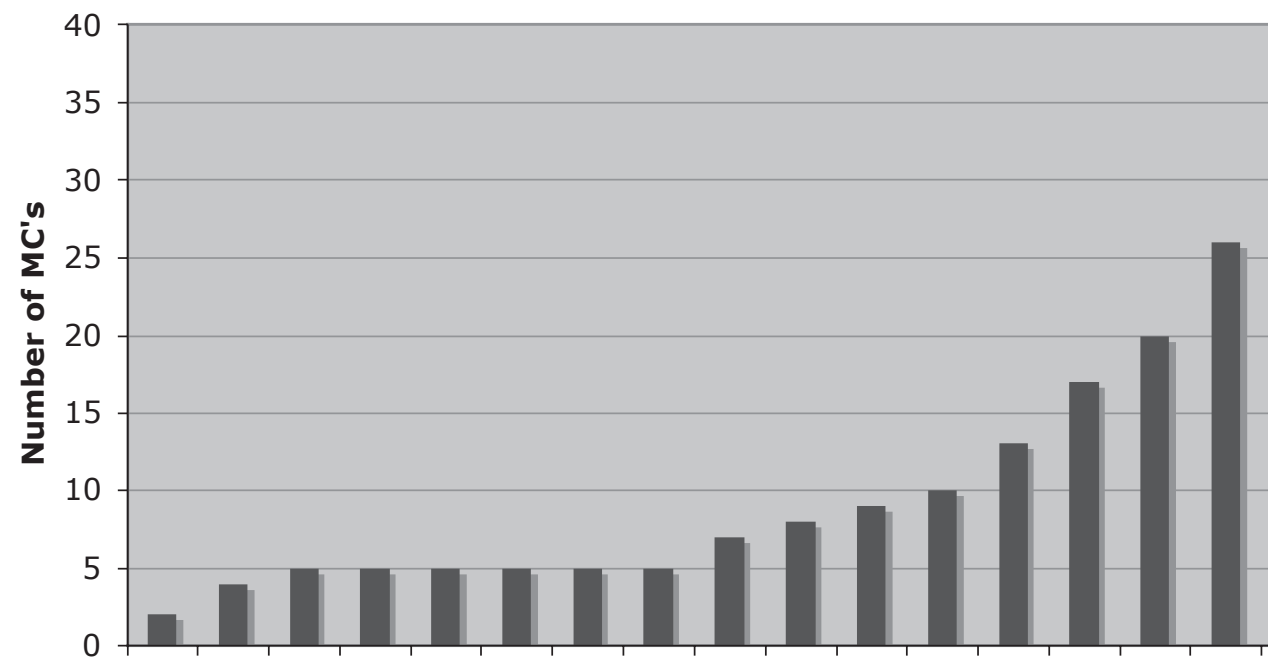

Figure 1: Starting year of memory clinics in the Netherlands, cumulative from $1986(\mathrm{~N}=35$, missing data of 5 settings). 
that were mainly associated with MC's were clinical geriatry (83 clinicians), (neuro)psychology (58 clinicians), neurology (53 clinicians), and psychiatry (35 clinicians). Besides this, also nurses played an important role.

In 2004, objectives were in more than $90 \%$ van the MC's specialized diagnostics, pharmacological treatment and giving advice and information. The majority of the settings also offered non-pharmacological treatments or gave advice about future policy ( $\pm 70 \%)$. These findings are similar to that in 1998. Half of the MC's also was involved in scientific research, and the minority (38\%) also had educational aims.

In the diagnostic phase, neuropsychological assessments were applied in $75 \%$ of the settings. Laboratory assessments were applied in $97 \%$ and all settings used imaging techniques, most commonly MRI-scan or CT-scan. All settings used one or more assessments scales. In 97\% of the settings the Mini Mental State Examination (MMSE) was used. Twenty-six settings (70\%) used one or more dementia screenings scales, such as the Alzheimer's Disease Assessment Scale-Cognitive Subscale (ADAS-COG) (8 settings), de Clinical Dementia Rating Scale (CDR) (10 settings) en de Global Deterioration Scale (GDS) (3 settings). Depression scales were used in $86 \%$ of the settings, mainly the Geriatric Depression Scale (GDS). Other scales used in MC's were the Neuro-Psychiatric Inventory (NPI(-Q)) (8 settings), Interview for Deterioration in Daily Living activities in Dementia (IDDD) (7 settings) en Instrumental Activities of Daily Living (IADL) (4 settings).
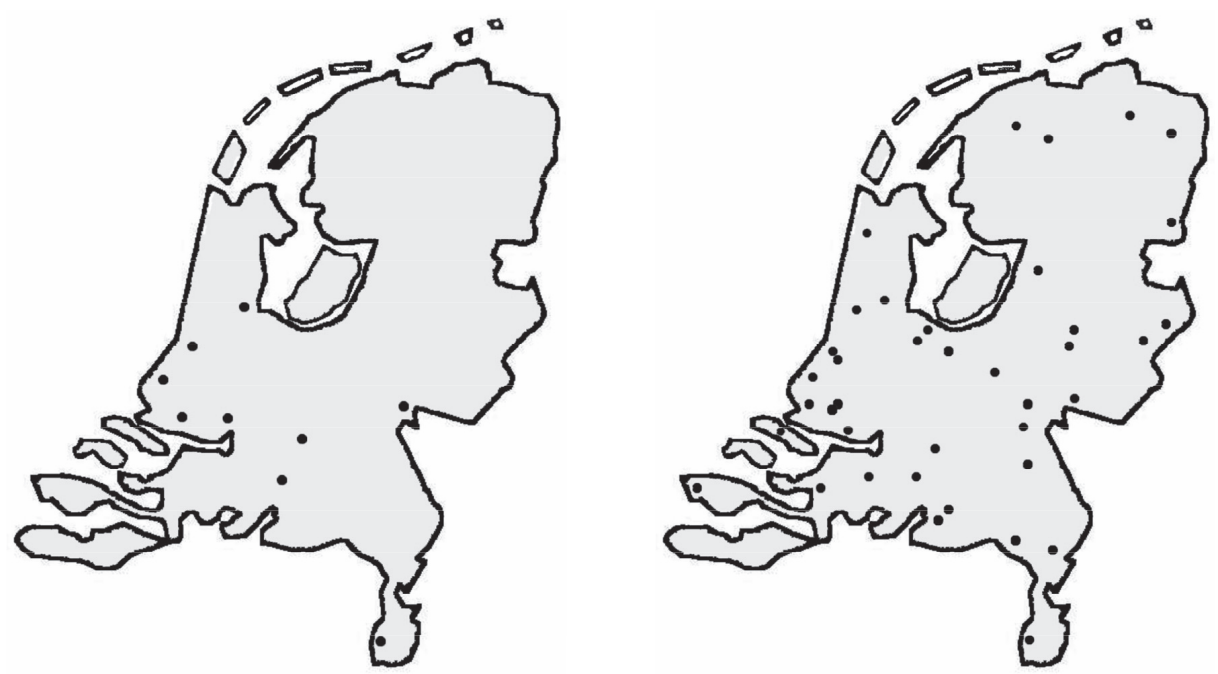

Figure 2: Topographical distribution of MC's: in 1998 (left) and 2004 (right). 
The diagnosis $\mathrm{MCl}$ was used in 34 of the 37 settings (92\%). The criteria $\mathrm{MCl}$ were defined by 30 settings. Nineteen settings used the amnestic criteria of Petersen et al. (1999). A combination of cognitive impairments was used in 8 settings, and 3 settings used other criteria. The average percentages of the diagnostic classifications of $\mathrm{MCl}$-cases are presented in Table 1. These data are based on the available data of 23 settings. The diagnoses 'primary degenerative,',vascular', and 'not specified' were most common.

The mean number of patients seen in 2003 per setting was 199 (range 15-615), compared to 130 in 1998. Sixty-two percent of the new patients in 2003 were diagnosed as demented (range 40-95\%) (Figure 3). This implicates that dementia is the most common syndromal diagnosis in MC's in the Netherlands. Besides dementia, a substantial part of the subjects $(23 \%$, range $5-45 \%$ ) had $\mathrm{MCl}$ (i.e. a cognitive impairment in memory and/or other cognitive domains). About 15\% (range $0-40 \%$ ) of the subjects had no cognitive disorders. There was no significant difference in the proportion of the syndromal diagnoses dementia/MCl/ no cognitive disorders among the coordinating disciplines neurology, psychiatry and clinical geriatrics, and also not between academical or not-academical settings. $38 \%$ of the settings saw for $70 \%$ or more subjects with dementia. In about one-fourth (26\%) of the MC's, more than half of the subjects did not get the diagnosis of dementia.

Pharmacological treatment was used in $97 \%$ of the MC's, not related to a clinical trial. All these settings prescribed cholinesterase-inhibitors. The main implication for prescribing cholinesterase-inhibitors was Alzheimer's disease. None of the settings reported $\mathrm{MCl}$ as an indication for prescribing cholinesterase-inhibitors. Psychosocial treatment was used in $65 \%$ of the settings. 19 settings (24\%) gave some type of cognitive training. Psychosocial interventions were more often part of the treatment protocol in settings coordinated by the geriatric $(83 \%)$ or psychiatric (100\%) department than in settings coordinated by the neurology department $(38 \%, p=.012)$. In 31 of the $37(84 \%)$ settings was indicated that the treatment of $\mathrm{MCl}$ patients

Table 1. Diagnostic classification of new MCl-patients

$\begin{array}{ll}\text { Diagnostic classification } & \text { Mean \% [min-max] } \\ \text { Primary degenerative } & 20[0-80] \\ \text { Cerebrovascular } & 20[0-80] \\ \text { Not specified } & 19[0-80] \\ \text { Depression } & 10[0-50] \\ \text { Somatic/internal cause } & 6[0-40] \\ \text { Other neurological cause } & 9[0-100] \\ \text { Intoxication/Side effects } & 3[0-15] \\ \text { Other psychiatric cause } & 2[0-10]\end{array}$


differed to that of demented patients. Patients with $\mathrm{MCl}$ were followed over time in 34 (92\%) settings. This mainly as a neuropsychological follow-up assessment, either in the kind of a scientific research project or not. A structural collaboration with local service providers for mental health increased from 15\% of the MC's in 1998 to $63 \%$ in 2004.

\section{Discussion}

MC's in the Netherlands had a strong development in the past years. Not only the number of settings, but also their total capacity showed a substantial increase. Nowadays, the MC's are more equally distributed with respect to the topographical location in the country, and there is more balance in the academic or non-academic settings. Besides subjects with dementia, also subjects with memory problems who are not demented are a substantial part of the subjects who attended MC's (Figure 3).

Nowadays, a substantial part of the demented patients are seen in a specialized service for the diagnostics of early dementia. The incidence of dementia in the Netherlands is about 22.500 patients per year (Ruitenberg et al., 2001). In 1998, less than $5 \%$ of the new demented patients were seen at a MC, compared to $22 \%$ in 2004. This indicates that MC's have outgrown the exclusive setting, that was mainly focused on scientific research, and have get a place in the regular care. This development is similar with that in foreign countries (Lindesay et al., 2002). The strong increase in the number of settings can be explained by the increasing focus on the early diagnosis of dementia and the registration of the first pharmaceutical treatments for Alzheimer's disease in the Netherlands, in 1998.

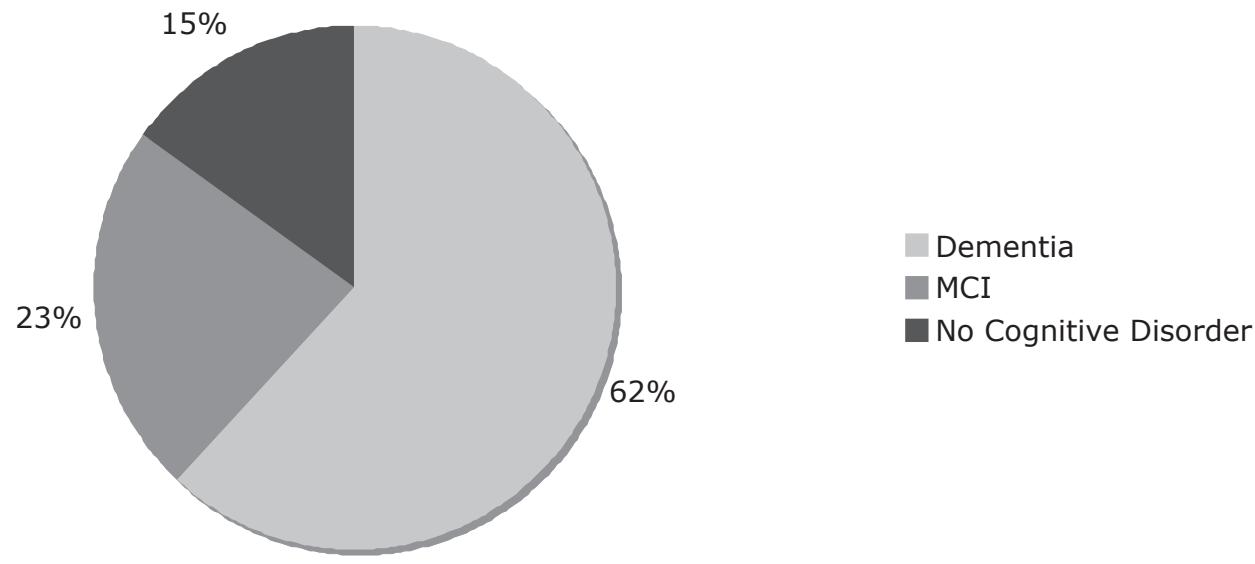

Figure 3: Distribution of diagnoses of new referrals to MC's. 
The majority of the settings used neuropsychological assessments and one or more screening scales for cognition and dementia, which is in line with the recommendations of an evidence-based review about the early detection of dementia (Petersen et al., 2001). However, there was a large variability in which specific scales were used. Harmonization about the cognitive domains that should be measured, the used neuropsychological tests or screening instruments and the used normative data of these tests would increase the comparability of the results between several settings.

MC's play an important role in the diagnostic process of dementia, its aetiology, and the explanation of this diagnosis to the patient and his family. MC's also give information about the implications of the diagnosis, the course, and advice about coping with the disease. However, a MC also has an important function in the diagnostic process of subjects with memory complaints who are not demented, as about $40 \%$ of the new referrals to a MC had $\mathrm{MCl}$ or no cognitive disorder. Remarkable is the large variability between the settings in the proportion of the diagnoses dementia, $\mathrm{MCl}$ and no cognitive disorder. This suggests that we can identify two types of MC's: those that see mainly demented patients, and those that see mainly milder types of memory disorders in the absence of dementia. The proportion of MC's that mainly saw demented subjects is decreased from 58\% in 1998 (Verhey et al., 1999) to $38 \%$ in the present study. This implicates that nowadays there is increasing emphasis for milder cognitive problems such as $\mathrm{MCl}$ and no cognitive disorders. As mentioned before, $\mathrm{MCl}$ is not always a preclinical phase of dementia, as the cognitive profile of these subjects can also remain stable or can even improve over time (Visser et al., 2002). Cognitive impairments that remain stable or improve over time will probably not be caused by underlying degenerative pathology, but probably by other factors that may cause cognitive complaints such as several psychological, behavioural or social factors, which emphasized the importance of an extensive neuropsychiatric screening in these subjects.

About $15 \%$ of the visitors of Dutch MC's did have no cognitive disorder. These subjects often experience cognitive complaints because of other factors. Previous research showed that memory complaints can be related to psychiatric symptoms, personality characteristics and poor physical health (Cargin et al., 2007; Comijs et al., 2002; Derouesne et al., 1999; Jorm et al., 2004; Ponds et al., 1997). Subjects with subjective cognitive complaints, but without impairments on formal testing are often referred to as 'worried well'. These subjects often worry about their complaints and are feared to become demented. Nevertheless, also these subjects seek help at MC's and should be taken seriously. MC's should also have treatment possibilities for this group of visitors, who are characterized by less memory self-efficacy, a decreased quality of life related to mental health, and more worries related to a family history of dementia (Ramakers et al., accepted). 
Pharmacological treatment was available in nearly all MC's. However, as none of the settings prescribed cholinesterase-inhibitors to subjects with $\mathrm{MCl}, \mathrm{MC}^{\prime}$ s that are mainly visited by subjects with $\mathrm{MCl}$ or no cognitive disorder should have other treatment possibilities than pharmacological treatment. This implicates that the treatment possibilities of a MC should be fitted to the problems of the visitors of that particular MC. The majority of the settings performed neuropsychological follow-up assessments in subjects with $\mathrm{MCl}$. Other starting points for non-pharmacological treatment in subjects with $\mathrm{MCl}$ or subjects with no cognitive disorder can be psychosocial treatment, cognitive training, or psycho-education to the patient and the family. $65 \%$ of the settings reported to have psychosocial treatment available. This is a clear increase since 1998 when only $38 \%$ of the settings could deliver psychosocial treatment (Verhey et al., 1999). This development is in line with nowadays standards (Draskovic et al., 2008), in which the availability of non-medication treatments such as psychosocial interventions is recommended as part of best MC practice. Psychosocial interventions were more often part of the treatment protocol in settings coordinated by the geriatric or psychiatric department than settings coordinated by neurologists. This suggests that the composition of the staff of the MC has implications for the available treatment possibilities.

The majority of the settings performed additional diagnostic methods, such as extensive neuropsychological assessments, imaging techniques, laboratory findings, or lumbar punctions, to investigate the aetiology of the cognitive impairments. This underlines the shift from a syndromal diagnosis to a more aetiological diagnosis at an earlier time point in the course of the disease. However, the use of these diagnostic markers should not implicate that clinical impressions and observations are less important and should move to the background.

Half of the MC's were involved in scientific research, which is a decrease of $35 \%$ since 1998 (Verhey et al., 1999). This implicates that scientific research nowadays is less limited to academical settings as it was in 1998. The role of scientific research at a MC is important, as MC's are good settings for the standardized inclusion of subjects along the whole cognitive spectrum, ranging from subjective cognitive complaints to dementia. MC's are also good settings for the implementation of new scientific findings and for investigating the effects of these implementations.

This inventory shows that MC's have a large consensus about the objectives, used diagnostic criteria for Alzheimer's disease and vascular dementia, and the used scales. However, differences between the MC's exist about the number of patients per week, and the partition of diagnoses of subjects with dementia and subjects with cognitive impairment without dementia. This inventory has reached the majority of the MC's in the Netherlands. However, there will be settings that have comparable objectives and activities, such as monodisciplinary settings, which did not participate in this inventory. This leads to an underestimation of some 
results. Another point of attention is that results are mainly based on estimations. Nevertheless, we suppose that this inventory is a representative overview of the current state of MC's in the Netherlands. There is an increasing emphasis on subjects with milder cognitive problems. MC's can and should be adequate settings for referrals along a broad cognitive spectrum, including $\mathrm{MCl}$ (independent from definition) and those with 'only' subjective memory complaints. There is an increasing number of people who want to know about their prognosis and future chance to becoming demented. Those who have these questions deserve an honest and evidence based answer. MC's should have diagnostics and expertise available to differentiate between the several underlying causes of cognitive impairments. For subjects who are at increased risk for developing dementia, MC's should have a monitoring function ( $3^{\text {rd }}$ CCCDTD, 2007). In addition, MC's should be able to give, by itself or in cooperation with other care facilities in the region, an integral treatment, that is not only focused on medication and that is fitted to the problems of his visitors.

In conclusion, these findings showed that the population of patients that visit a MC's is not limited to demented patients, but also subjects with more mild cognitive problems are a substantial part of the visitors to MC's. Because of the aging population and when new treatment possibilities to slow down the disease progression become available, the number of subjects with mild cognitive problems that seek help at a MC will increase. The treatment arsenal of MC's should be fitted to this group of subjects, and should not be limited to the pharmacological therapy for dementia. 
Concluding remarks 


\subsection{Introduction}

Mild Cognitive Impairment $(\mathrm{MCl})$ is used to describe the transitional phase between normal cognitive functioning and dementia. In this phase subjects experience cognitive problems, that do not meet the current criteria for dementia. Subjects with $\mathrm{MCl}$ are at increased risk for developing dementia, especially AD (Geslani et al., 2005; Petersen et al., 1999; Visser et al., 2006). However, not all subjects with MCI will develop AD (Visser et al., 2002). It is important to identify predictors that can differentiate between subjects who will develop $A D$ and those who will not, as both groups can benefit from other types of advice and treatment.

The aim of the first part of the thesis was to investigate predictors for AD or dementia in a memory clinic and GP setting. In Chapters 2 and 4, we investigated the predictive accuracy of cognitive and non-cognitive variables for $A D$ in subjects with $\mathrm{MCl}$, selected from a memory clinic setting. In addition, we studied the predictive value of cognitive, affective, behavioural, and vascular symptoms, gait disturbances, and changes in appetite and weight for future dementia in subjects from general practice settings (Chapter 5).

The aim of the second part of the thesis was to investigate the development and organization of Memory Clinics, as a relatively new health care facility for subjects with cognitive problems. We investigated determinants of help-seeking behaviour for subjective memory complaints at a memory clinic (Chapter 6). In addition, we studied the development of memory clinics in the Netherlands since 1998, and we focused on the role of this specialized health care facility for non-demented subjects with $\mathrm{MCl}$ or no cognitive disorder (Chapter 7). In this last chapter of the thesis, we will summarize and discuss the general findings. We will also describe the methodological considerations, as well as the scientific and clinical relevance of these findings. Lastly, recommendations for future research will be made.

\subsection{Predictors of Alzheimer's disease}

\subsubsection{Memory}

It is well known that $A D$ is characterized by impaired memory functioning. Non-demented subjects with memory impairments are at increased risk for developing AD (Albert et al., 2007; Sarazin et al., 2007; Saxton et al., 2004; Tabert et al., 2006; Twamley et al., 2006; Visser et al., 2002). Therefore, decreased memory performances may be an early marker of AD. The measure generally used for investigating the predictive accuracy of memory for $A D$, is delayed recall performance. However, delayed recall is the result of a variety of processes, such as encoding, storage and retrieval. Thus, other aspects of memory functioning may also be indicative of early AD. 
Findings of the present study showed that the use of an active memory strategy during the learning of verbal information correlated with delayed recall performance and was also a significant predictor for future $A D$, as lower scores of subjective organization were associated with a higher risk for $A D$ up to 10 years after baseline (Chapter 2). The more passive learning strategy of serial clustering had no predictive value. In addition, we found that this effect was similar in middle-aged subjects (aged 40-59 years) and older subjects (aged 6085 years). The finding that prodromal $A D$ is characterized by impaired learning strategies may have implications for the early detection of $A D$ in subjects with $\mathrm{MCl}$. These findings are consistent with the idea that strategies, which are more effortful for the total cognitive brain capacity, are more sensitive to degeneration than less effortful, more automatic strategies. These findings give an insight into how the subject has reached the total score. Subjective organization depends on the active reorganization of the incoming information, and may therefore be related to prefrontal lobe functioning and executive functioning (Fletcher et al., 1998; Fletcher et al., 1998; Kramer et al., 2005; Savage et al., 2001). We did, however, not find evidence that learning strategies were dependent on executive functioning, because after correction for measures of executive functioning (Stroop III score, TMT B score and fluency), results did not change. The finding that the use of an active learning strategy during the 5 immediate recall trials is strongly correlated with the performance on the delayed recall can be used in the development of cognitive training programs, where subjects with memory complaints may learn to use learning strategies to enhance everyday independent memory functioning. Recent studies have shown that subjects, also at an older age have the capability to learn and that $\mathrm{MCl}$ subjects can benefit from memory training programs (Belleville, 2008). Nevertheless, the benefits of these training programs will be most effective in early phases of the degenerative process.

In Chapter 3, we investigated the relation of several aspects of verbal memory and learning strategies with the APOE genotype in subjects with $\mathrm{MCl}$. We found that in younger subjects impairment in the active memory strategy were related to the APOE e4 genotype, a well-known genetic risk factor for AD, whereas in older subjects the APOE e4 allele was most strongly related to impaired delayed recall performances. After the exclusion of subjects who developed AD at follow-up, the APOE e4 allele remained associated with lower levels of subjective organization in the middle-aged subgroup, but was no longer associated with any of the memory measures in the older subjects. Thus, the effect of the APOE e4 genotype on memory function may be dependent on underlying AD pathology in elderly subjects, but not in middle-aged subjects. This suggests that the APOE e4 allele may have an independent mechanism on memory functioning in middle-aged subjects with $\mathrm{MCl}$, or that these subjects already suffer from $A D$, but that the follow-up period was too short to identify conversion to 
CHAPTER 8

$A D$ in these middle-aged subjects. In that case, subjective organization could be a very early marker of AD.

\subsubsection{Affective symptoms}

Besides cognitive problems, subjects with $\mathrm{MCl}$ can also experience non-cognitive symptoms, such as affective symptoms (Apostolova \& Cummings, 2007; Copeland et al., 2003; Gabryelewicz et al., 2007; Geda et al., 2004; Liu et al., 2007; Modrego \& Ferrandez, 2004; Palmer et al., 2007; Panza et al., 2008; Robert et al., 2006; Rozzini et al., 2005; Teng et al., 2007; Tierney et al., 1999). There are several possible explanations for the relation between affective symptoms, cognitive impairment and AD. Affective symptoms and $\mathrm{MCl}$ can be caused by an underlying depression and in that case affective symptoms would be associated with a decreased risk for $\mathrm{AD}$. Affective symptoms and $\mathrm{MCl}$ may also be associated with underlying $\mathrm{AD}$ pathology and then affective symptoms would be associated with an increased risk for AD. It has been suggested that affective symptoms are risk factors for AD (Modrego \& Ferrandez, 2004; Ownby et al., 2006; Palmer et al., 2007). However, there is no consensus about the predictive role of these symptoms for $A D$ in subjects with $\mathrm{MCl}$, as other studies found no relation or found a decreased risk for AD (see Table 3, Chapter 4 for an over view). These conflicting findings may have resulted from several factors. In Chapter 4, we have investigated possible explanations for these discrepant findings such as age, length of follow-up, and definition of $\mathrm{MCl}$.

Our findings showed that indeed affective symptoms are common in subjects with $\mathrm{MCl}$, but that these symptoms are not a marker of $A D$ in the predementia stage, as these symptoms in general were associated with a decreased risk for AD (Chapter 4). The finding that affective symptoms are common in subjects with $\mathrm{MCl}$ is in line with other studies. The prevalence of affective symptoms in our study (50-70\%) is in line with a recent review that reported behavioural symptoms in $35-75 \%$ of the $\mathrm{MCl}$-subjects with the most common being depression, anxiety, apathy and irritability (Apostolova \& Cummings, 2007). The observed differences in the prevalence of affective symptoms between other studies and the present one can be explained by different sampling methods, different settings, differences in the operationalisation of $\mathrm{MCl}$, and the instruments used to measure affective symptomatology.

With respect to the predictive value of affective symptoms, we found that sleeping problems were significantly associated with a lower risk for $A D$, and that symptoms of depression and anxiety showed a trend in this direction. Thus, affective symptoms in subjects with $\mathrm{MCl}$ seem not to be caused by underlying $A D$ pathology, and therefore cannot be used in clinical practice to identify subjects who are at increased risk for AD. As shown in table 3 , Chapter 4, these findings are in line with several other studies which also found no or a negative association between depression and AD (Korf et al., 2004; Panza et al., 2008; Teng 
et al., 2007; Visser et al., 2000; Wang et al., 2006), however, some other studies did found an increased risk (Gabryelewicz et al., 2007; Modrego \& Ferrandez, 2004). We could not find a clear explanation for these inconsistent results among the studies. The type of $\mathrm{MCl}$ may have an effect on the association between depression and AD, as we found that depression was significantly associated with a decreased risk for $A D$ in subjects without amnestic $\mathrm{MCl}$, but was not associated with $\mathrm{AD}$ in subjects with amnestic $\mathrm{MCl}$. We showed that age and the length of follow-up did not have a major influence on these findings. The predictive value may also depend on type of rating scale and setting. We used a clinician based rating scale. However, the use of a self-rating scale for depression and anxiety (Arrindell \&Ettema, 2003; Arrindell \& Ettema, 1981) would have yielded similar results (data not shown). The setting from which the subjects were selected was the Maastricht Memory Clinic, which is embedded in the Department of Psychiatry of the hospital. It may be possible that our sample is relatively enriched by subjects with primary depression. Despite the lack of usefulness of affective symptoms for identifying subjects with preclinical AD in clinical practice, the high prevalence of these symptoms require clinical expertise of affective symptomatology in diagnostic and treatment settings for subjects with $\mathrm{MCl}$.

\subsubsection{Predictors in GP settings}

The GP plays an important role in the early identification of subjects with preclinical dementia. However, knowledge about which symptoms are suspected for preclinical dementia in general practice settings is very limited and may cause a delay in time between the presence of the first symptoms and the diagnosis. In our study (Chapter 5), we found that 5 years prior to diagnosis, subjects with preclinical dementia visited their GP more often than controls. Rather unexpected, gait disturbances were the earliest predictor of a dementing disorder. Cognitive complaints were predictive for dementia in the three years before diagnosis. In the year prior to diagnosis cognitive, affective, and behavioural symptoms, gait disturbances and changes in weight and appetite were predictive for dementia. Vascular symptoms were not predictive in any of the years prior to diagnosis. We found that $58 \%$ of the subjects with preclinical dementia reported cognitive complaints to their GP in the year prior to diagnosis. These data are of interest for identifying subjects at increased risk for dementia, at the same time they imply that $42 \%$ of the subjects did not report these complaints to the GP in the year before the diagnosis of dementia was made. This indicated that the GP should play an active role in the investigation of cognitive problems in older subjects, in which the GP should not only wait until the patient contacts him with cognitive complaints, but that the GP should inventorise these symptoms in a pro-active way to identify those subjects who are at risk for future dementia, and who also could benefit form additional diagnostics. 
The findings of this chapter seem not to be in line with findings from Chapter 4, in which we investigated the predictive value of affective symptoms in subjects with $\mathrm{MCl}$ selected from the memory clinic. Findings showed that the presence of affective symptoms were associated with a decreased risk for $A D$ in subjects selected from the memory clinic, but with an increased risk for dementia in subjects from the GP settings. The difference may be explained by differences in mean age of the subjects, as subjects from the GP-setting were older than subjects from the memory clinic. Also the method of assessment of the affective symptoms differed in both studies, which may have resulted in a higher prevalence of affective symptoms in the sample selected from the memory clinic compared to the GP-sample.

\subsection{Memory clinics}

\subsubsection{The development of memory clinics and their role for subjects with $\mathbf{M C l}$}

MCs are relatively new facilities in health care. The first MC in The Netherlands developed in 1986 in Maastricht and Tilburg (Verhey et al., 1999). Findings from chapter 7 show that nowadays, memory clinics play an important role in the health care system in the Netherlands. They have had a strong development in the past years, as there was a strong increase in the number of settings, and their total capacity. The most common syndromal diagnosis was dementia. They also became important facilities for subjects without dementia, such as subjects with $\mathrm{MCl}$ or subjects with "only" subjective memory complaints, as these subjects make up about $40 \%$ of their visitors. Also because of their multidisciplinary way of working, MCs should be able to investigate all possible causes which underlie cognitive complaints and to deliver diagnostics and treatment possibilities among a broad continuum of cognitive problems.

\subsubsection{Seeking help at a memory clinic}

Subjective memory complaints (SMC) defined as memory complaints in the absence of objective impairments on memory tests, are common in middle-aged and elderly subjects. Only a subset of subjects with SMC decides to seek medical attention for these complaints. It is important to investigate the characteristics of these help-seeking subjects to determine the needs of these subjects and to provide adequate treatment and advice. Compared to subjects who do not seek help for their SMC, subjects who seek help at a memory clinic for their SMC are characterized by having more negative beliefs about their personal competence in various memory situations (low memory self-efficacy), low quality of life related to mental aspects, more deterioration as experienced by a close relative, and that these subjects are more often worried about dementia because of a family history of dementia (Chapter 6). This information is important as these subjects form a substantial part (15\%) of the visitors of (Dutch) MCs 
(Chapter 7). These subjects often worry about their forgetfulness and want a prognosis. MCs should also have diagnostics and treatment tools available for this group of MC-visitors. Also, in the absence of an objective memory impairment, subjects with SMC who seek help at a MC should be taken seriously, as a progressive course of the cognitive complaints caused by underlying degenerative pathology cannot be excluded in a cross-sectional assessment. This is confirmed by findings of Chapter 6 , which showed that $11 \%$ of the subjects with subjective memory complaints without impaired memory scores from the $\mathrm{MC}$ had developed dementia at follow-up.

\subsubsection{Methodological considerations}

The present thesis showed findings from several study designs, such as a prospective, longitudinal design (Chapter 2 and 4), a retrospective cohort study (Chapter 5), cross-sectional studies (Chapters 3 and 6), and an inventory (Chapter 7).

\subsubsection{Strengths}

Strengths of the Maastricht MCl study (Chapters 2-4) are its prospective, longitudinal design, in which subjects who fulfil the inclusion criteria were selected at baseline and were followed over time. This prospective design prevents selection biases. Another strength is the long follow-up period up to 10 years. To our knowledge, there is only one other study with a follow-up period up to ten years, which is however a community study (Ganguli et al., 2004). This implies that this follow-up period is unique in clinical research about $\mathrm{MCl}$. A follow-up period of 10 years will have identified the main part of the converters. Another strength of the present study was the large sample size, which allows reliable findings and which enables it to apply multivariate statistical analyses. Also the clinical settings, from which the subjects were selected, make the findings of this thesis relevant for clinical practice. In addition, we presented findings from GP-settings (Chapter 5 ) investigated with a case-control design matched for age, sex and GP setting. For this study, we used data from the RNH database (Metsemakers et al., 1992). This is a large database, which at the time of the data selection contained data from 21 rural and urban GP practices. The RNH database is a well-documented database, in which patient characteristics and all relevant health care problems are stored. The register offers a unique opportunity as a sample frame for research in a general practice setting on which crosssectional and longitudinal analyses can be performed (Metsemakers et al., 1992). In Chapter 6, we compared clinical data from subjects from a memory clinic setting to population-based data from the Maastricht Aging Study (MAAS) (Jolles et al., 1995). The MAAS is a large longitudinal population-based study into the determinants of cognitive aging and was performed in the same geographical region as the Maastricht Memory Clinic. 


\subsubsection{Loss to follow-up}

On the other side, the study described in this thesis also had several methodological problems. Firstly, as in any longitudinal study loss to follow-up is a problem as loss to follow-up may not be random. In our study the loss to follow-up was 15 to $18 \%$ (Chapters 2 and 4). In order to analyze whether the loss to follow-up had biased our results, we compared subjects with and without missing data (outcome) at follow-up. The findings of Chapter 4 showed that in the study population of subjects older than 55 years, there were no differences in baseline characteristics between subjects with and without outcome at follow-up. The findings of Chapter 2 showed that in the total study sample (40 years or older), subjects without outcome at follow-up were about three years older, and had significantly lower scores on the GDS and BDS. This implied that the findings of the study were based on somewhat younger subjects who had a higher level of functioning than the total $\mathrm{MCl}$ population from the memory clinic. As subjects who are older and have a greater decline in functioning are at higher risk for developing $A D$, findings with respect to conversion rates will be slightly underestimated. However, our conversion rates to $A D$ in subjects with $\mathrm{MCl}$ was $11 \%$ in the first two years (Chapters 2 and 4), which is in agreement with the mean conversion rate reported in a recent review about conversion rates to $\mathrm{AD}$ in $\mathrm{MCl}$ subjects of $10.2 \%$ per year (Bruscoli \& Lovestone, 2004).

\subsubsection{Follow-up study design}

The Maastricht $\mathrm{MCl}$ study was an ongoing study. This implied that not all subjects could have had a follow-up assessment after 5 or 10 years, because they did not participate for long enough in the study. This resulted in smaller sample sizes at the 5-years follow-up and at the 10-years follow-up, and may have influenced the statistical power. We had follow-up assessments after 2, 5 and 10 years. This implicates that AD is diagnosed at one of these time-points, but that the subjects may have suffered from it earlier. Thus, the precise date of the diagnosis is not available. Therefore, we used a discrete-time survival analysis model (Hosmer \& Lemeshow, 1999), which was implemented using logistic regression and an appropriately adjusted dataset (Hosmer \& Lemeshow, 1999) to investigate the predictive accuracy of the cognitive (Chapter 2) and affective symptoms (Chapter 4 ) after 2-years, 5-years and 10-years. This technique analyzes time in discrete chunks during which $A D$ could occur.

\subsubsection{Generalizibility}

Another point of attention is the generalizibility of our results. Our studies with subjects with $\mathrm{MCl}$ were conducted in a memory clinic of a university hospital. The university hospital of Maastricht combines an academic and a regional setting, which makes the study sample representative for clinical settings. However, the findings based on this sample may not be 
applicable to community-based samples. The Maastricht memory clinic is embedded in the psychiatry department of the hospital. Therefore, the findings based on this sample may not be totally applicable to settings that are based on neurological or geriatric departments, as these may differ in mean age of the subjects, severity of the cognitive impairments, and comorbidities, such as affective or vascular problems.

We also performed a study in settings of GP-practices, including subjects selected from the Registration Network of Family Practices (RNH). The RNH is computerized and anonymous database that contains all relevant health care problems of rural and urban GPs in the Southern of The Netherlands (Metsemakers et al., 1992). As it can be assumed that every inhabitant of The Netherlands has a GP, this database is an adequate reflection of the Dutch population.

\subsection{5 $\mathrm{MCl}$ definition and concept}

In the literature many definitions can be found to describe the transitional phase between normal cognitive functioning and dementia. The use of different definitions has an impact on prevalence and incidence rates, and on conversion rates for AD. In addition, the used definition has to be taken into account in the generalizibility of the results.

On the one hand, there is variation in the cognitive domains involved. Some of these definitions focus only on memory impairments, such as Petersen's MCl (Petersen et al., 1999), while others include also impairments in other cognitive domains, such as attention, concentration, language or executive functioning for example Cognitive Impairment No Dementia (Graham et al., 1997)).

On the other hand, there is variation in the definition of an 'impairment'. Some researchers mention that these impairments can be reported subjectively by the subject and/ or a relative (GDS; (Reisberg et al., 1982)), or can be a clinical judgement, while others require that these impairments are objectified by impaired test performances, of which again there is no consensus about the cut off scores.

In addition, the usefulness of $\mathrm{MCl}$ in clinical practice is still under debate. Some researchers state that $\mathrm{MCl}$ represent a useful clinical entity (Petersen et al., 2004). However, criticisms of the $\mathrm{MCl}$ concept call $\mathrm{MCl}$ a heterogeneous concept and an unreliable term, and are sceptic about using $\mathrm{MCl}$ as a clinical entity. They challenge the use of criteria originally developed for research purposes as a diagnosis in clinical practice (Whitehouse \& Moody, 2006). However, nowadays more and more subjects with $\mathrm{MCl}$ ask for clarity about the nature of their complaints and want to get a prognosis. These subjects deserve an honest, reliable, and valid answer to their questions. The definition, nature and clinical usefulness of $\mathrm{MCl}$ will remain an area of interest in aging research. However, whatever the preferred definition may be, the 
presence of having mild cognitive problems, makes subjects at increased risk for developing $A D$ in future.

In this thesis, we used $\mathrm{MCl}$ to refer to subjects, who had subjective memory complaints in the absence of dementia, and had a GDS score of 2 or 3, and no significant impairment in general cognitive functioning or daily functioning. This definition is broader than the original MCl-criteria of Petersen and colleges (Petersen et al., 1999), which require a cognitive impairment objectified on formal testing. However, we chose to use this broad definition to investigate the whole cognitive spectrum between subjective cognitive complaints and dementia. Also other studies have used these criteria (Feldman \& Jacova, 2005; Tabert et al., 2006; Tierney et al., 1996). The use of a broad definition of MCl leads to higher sensitivity for $A D$, but specificity will be lower, as more subjects included in our study that had cognitive problems due to reasons other than brain degeneration caused by $A D$ compared to studies that used the more restricted amnestic $\mathrm{MCl}$ criteria. However, in research about early markers for $A D$ it is important to include a broader cognitive range, as previous studies found that even very mildly impaired individuals are at increased risk for AD within about 5 years (Dickerson et al., 2007). Post-hoc analyses in the memory clinic sample showed that the exclusion of subjects without an objectified memory impairment at baseline would in the present study result in the lack of detection of $26 \%$ of the subjects who convert to AD.

\subsection{Recommendations}

\subsubsection{Scientific implications}

Based on our findings, we have some recommendations for future scientific research. The use of effortful memory strategies has shown to be a significant predictor for AD. Further research should investigate the relation between memory strategies and other cognitive measures and between memory strategies and other biological aspects, such as imaging and cerebrospinal fluid markers. Studies into the preclinical phase of dementia should not be restricted to subjects with impairments on formal memory tests as the present study showed that this would have lead to the lack of detection of a substantial number of the subjects with future dementia. Also, other studies have shown that even subjects with only cognitive complaints (Dik et al., 2001; Jonker et al., 2000; Saykin et al., 2006) or very mildly impaired subjects (Dickerson et al., 2007) are at increased risk for developing AD. Another finding, which should be recommended for future research is the inclusion of subjects with affective symptomatology at baseline. Affective symptomatology is common is subjects with $\mathrm{MCl}$. We reported prevalence ranging from 50 to $70 \%$ in our population (Chapter 4). Because this prevalence is also high in subjects who converted to AD over time, the exclusion of these symptoms on forehand would also lead 
to the lack of detection of a substantial number of the subjects at risk. We also recommend a follow-up period of 5 to 10 years, depending on the severity of the cognitive impairments at baseline. Studies that include subjects with milder cognitive problems should have longer follow-up periods, because the time to conversion will take longer in these subjects compared to subjects with more prominent cognitive impairments. In the present study a substantial number (12\%) of the subjects with AD had converted at the 10-years follow-up (Chapter 4), and this percentage will be an underestimation because of the ongoing study design.

\subsubsection{Clinical implications}

The clinical relevance of the main findings are that the risk factors investigated in the first part of this thesis are helpful in the identification of $\mathrm{MCl}$ subjects who are at risk for developing AD. In a general practice setting gait disturbances are the earliest prodromal signs of dementia and cognitive symptoms were the strongest predictor for dementia. The presence of these symptoms should make the GP aware of a possible underlying dementia process and the GP should consider whether additional diagnostics or a referral to specialized facilities would give more clarity. Remarkably $42 \%$ of the subjects did not report cognitive symptoms in the year prior to the diagnosis of dementia. This highlights the importance of an active participation of the GP in clarifying complaints and problems, to identify subjects at risk. Identified risk factors in subjects from a memory clinic setting were impairments in quantitative memory scores, but also impairments in qualitative memory performances such as decreased use of effortful memory strategies during learning. Also, the finding that the use of memory strategies during learning improved long-term recall performances could be used in memory training programs to enhance memory functioning in daily life. Affective symptoms, on the other hand, seem not to be useful in the identification of $\mathrm{MCl}$ subjects at risk for AD. Nevertheless, the prevalence of affective symptoms in subjects with $\mathrm{MCl}$ was high. This implies that subjects with $\mathrm{MCl}$ are not only characterized by cognitive impairments, but also by non-cognitive aspects, such as affective symptomatology. This highlights the importance of a multidisciplinary approach in the diagnostics and treatment possibilities for subjects with $\mathrm{MCl}$.

This multidisciplinary approach should be present in memory clinic settings as described in Chapter 7. This study showed that about one quarter of the visitors to a memory clinic had $\mathrm{MCl}$. Another $15 \%$ of the memory clinic visitors had no cognitive disorder. Taken together these findings imply that a substantial number of MC-visitors turned out not to be demented. Because of the multi-causality of memory complaints in subjects with $\mathrm{MCl}$ or no cognitive disorders, MC's should also have diagnostics and treatment possibilities available for this group of subjects. With no current pharmacological therapy available, the treatment possibilities for these non-demented subjects should have other starting points such as 
psychosocial treatment, cognitive training or psycho-education. These non-pharmacological treatments can be further specified to findings of Chapter 6 , which showed that subjects with no cognitive disorders who visit the memory clinic for subjective memory complaints are characterized by lower memory self-efficacy, lower quality of life related to mental aspects, and more often worries about their forgetfulness because of a family history of dementia. This knowledge should be used in the development of interventions for memory clinic visitors who have subjective memory complaints.

\subsection{Conclusion}

In the present thesis, we increased the knowledge about predictive and diagnostic aspects for prodromal AD in memory clinic and GP practice settings. It is important to differentiate early between progressive and non-progressive $\mathrm{MCl}$, as both groups can benefit from other types of advice and treatment. Early recognition of a neurodegenerative disorder starts in the GP practice and the diagnosis may be clarified by specialized services, such as multidisciplinary memory clinics. We found that cognitive, affective, and biological predictors are important to consider in the early identification of subjects at risk. However, we are aware that not all possible predictors for $\mathrm{AD}$ in subjects with $\mathrm{MCl}$ were investigated in this thesis. It seems likely that a multi-factorial model including cognitive, non-cognitive, and biological predictors will optimize the early recognition of subjects with prodromal AD. 
Summary 
Problems in cognitive functioning are common in middle-aged and older subjects. Whether these cognitive problems are early symptoms of $A D$ or not remains an important question. The transitional phase between normal cognitive functioning and dementia is often called Mild Cognitive Impairment $(\mathrm{MCl})$. In the last 20 years, there has been a lot of interest in subjects with $\mathrm{MCl}$, because these subjects are at increased risk for developing AD. However, not all subjects with $\mathrm{MCl}$ will develop $\mathrm{AD}$ in the future. To be able to differentiate between subjects who will develop $A D$ and those who will not, it is important to identify predictors for future $A D$ in subjects with cognitive problems. In the first part of this thesis, the predictive value of several cognitive and non-cognitive factors for AD was investigated. In the second part, we focused on the development of memory clinic settings in the Netherlands and on the characteristics of subjects who seek help at a memory clinic for subjective memory complaints.

In Chapter 2, the predictive accuracy of learning strategies for AD was investigated in subjects with $\mathrm{MCl}$ from the Maastricht memory clinic. Subjects were reassessed over a period of 10 years. We found that effortful strategies, such as subjective organization, which requires an active reorganization of the presented information, was a significant predictor for future $A D$ in subjects with $\mathrm{MCl}$. The more passive serial clustering, on the other hand, was no predictor for AD. These effects were similar in middle-aged and older subjects. This finding may help to identify subjects with prodromal $A D$ in subjects with $\mathrm{MCl}$. We also found an association between the use of subjective organization during the immediate recall trials and the delayed recall performance. This information can be used in the development of cognitive training programs.

In Chapter 3, we investigated the relation between the Apolipoprotein (APOE) e4 allele and memory dysfunction in middle-aged and older subjects with $\mathrm{MCl}$ from the Maastricht memory clinic. In middle-aged subjects, the APOE e4 allele was most strongly related to decreased subjective organization and in the older subgroup to a decreased delayed recall performance. After excluding subjects with incipient $A D$, results remained similar in the middle-aged subgroup, but there was no longer an association between the APOE genotype and any of the memory measures in the old subgroup. We concluded that the APOE e4 allele is associated with impaired memory functioning, but that the memory measure affected varies with age. We also concluded that the effect of the APOE e4 allele on memory function may be dependent on underlying AD pathology in elderly subjects, but not in middle-aged subjects. This could mean that the APOE e4 allele directly affects memory functioning, independent of underlying $A D$ pathology, or that the follow-up period was not long enough for middleaged subjects to identify everyone who would progress to clinical AD. In that case subjective organization could be a very early marker of AD. 
In Chapter 4, the predictive value of affective symptoms for AD was investigated in subjects with $\mathrm{MCl}$ selected from the Maastricht memory clinic. We focused on symptoms of depression, anxiety, apathy, sleep problems, and somatic problems as measured with the HDRS. The follow-up period was up to 10 years. At least one follow-up was available for 228 subjects. Seventy-nine subjects developed AD. At baseline, affective symptoms were present in $50-70 \%$ of the $\mathrm{MCl}$ subjects. Sleeping problems were associated with significantly lower risk for AD. Symptoms of depression and anxiety showed a trend in the same direction. The presence of amnestic $\mathrm{MCl}$ modified the relation between depressive symptoms and the risk for $A D$, as depression was associated with a decreased risk in subjects without amnestic $\mathrm{MCl}$, but not in subjects with amnestic $\mathrm{MCl}$. Age and length of follow-up did not modify the risk of affective symptoms for AD. We concluded that affective symptoms are not diagnostic for prodromal $A D$ as these symptoms are associated with decreased risk for AD. In table 3 of this chapter, an overview is given of previous studies that established the predictive value of depressive symptoms for $A D$ in subjects with $\mathrm{MCl}$. The pooled $\mathrm{OR}$ of these studies indicates that depression is not predictive for $A D$ in subjects with $\mathrm{MCl}$. However, given the high prevalence of these symptoms in prodromal $A D$, the presence of these symptoms does certainly not exclude the possibility of prodromal AD. Because affective symptoms are common in subjects with $\mathrm{MCl}$, services for subjects with cognitive complaints should pay attention to this symptomatology, and should have psychiatric expertise available to diagnose and treat these symptoms.

In Chapter 5, we investigated the predictive value of cognitive impairments, affective symptoms, behavioural problems, vascular problems, gait disturbances and changes in weight and appetite for dementia in subjects from the GP registration network (RNH). We compared 74 subjects with dementia to 125 age and sex matched control subjects from the same GP setting. In this study, we investigated a period of five years prior to the diagnosis of dementia. In the five years prior to the diagnosis of dementia, subjects with preclinical dementia visited their GP more often than control subjects. Gait disturbances were the earliest predictor for dementia. Cognitive complaints were predictive in the three years before the diagnosis of dementia, with the strongest predictive value in the year before the diagnosis. In the year before the diagnosis $58 \%$ of the subjects with preclinical dementia reported these symptoms to their GP compared to only $2 \%$ of the control subjects. All other symptoms, except vascular symptoms, were predictive in the year prior to diagnosis. We concluded that preclinical dementia is associated with an increased contact frequency between patient and GP at least five years prior to the diagnosis of dementia. Gait disturbances and cognitive complaints are the earliest symptoms of preclinical dementia in GP practice. Given that almost half of the subjects with preclinical dementia did not report cognitive symptoms in the year prior to the diagnosis, we contend that the GP must play an active role in identifying subjects at risk for dementia. 
In Chapter 6, we investigated which factors determine why people with subjective memory complaints (SMC) seek medical attention. We compared 33 cases with SMC who sought help at a memory clinic to 85 control subjects with SMC from a population-based study who did not seek help for their memory complaints. All cases and control subjects did not have an objectified impairment on formal memory tests and both groups had comparable MMSE scores. We investigated symptoms of depression, anxiety, extraversion and neuroticism, memory self-efficacy, quality of life, changes in memory and daily functioning according to a relative, life-changing events, and a family history of dementia. Cases who sought help for SMC had lower memory self-efficacy and lower quality of life related to mental aspects. Relatives of cases reported more deterioration in daily functioning than relatives of control subjects and cases were more often worried due to a positive family history of dementia. Levels of depression, anxiety, extraversion and neuroticism were comparable in cases and control subjects. We concluded that subjects who seek medical attention for SMC are characterized by lower memory self-efficacy and quality of life, more deterioration in daily functioning and worries due to a family history of dementia, compared to subjects who did not seek help for SMC. These findings are important for the development of adequate interventions for subjects without objective memory impairment who visit a memory clinic for memory complaints.

In Chapter 7, we investigated the development of memory clinics (MCs) in the Netherlands. In addition, we studied whether, and how these facilities can play a role in nondemented subjects, such as subjects with $\mathrm{MCl}$. We found that since 1998, the number of MC's increased from 12 to 40 and that the number of referrals per service has also increased. Nowadays, the focus is less exclusively on academic centres. Dementia was the most important syndromal diagnosis (62\%), but also milder cognitive problems, such as $\mathrm{MCl}(23 \%)$ and no cognitive disorder (15\%) were common. Compared to 1998, current there is increasing emphasis for milder cognitive problems. There was a large variability between the settings in the proportion of the diagnoses dementia/ $\mathrm{MCl} /$ no cognitive disorder. The concept $\mathrm{MCl}$ was used in $92 \%$ of the settings. The most common diagnostic classification of subjects with $\mathrm{MCl}$ were primary degenerative, vascular and not specified. The treatment of subjects with $\mathrm{MCl}$ was in $84 \%$ of the settings different to the treatment of demented subjects. The conclusion was that MCs are nowadays part of the standard care for subjects with dementia. Besides this, MCs play also an increasing role in the diagnostic and therapeutic procedures of subjects with milder cognitive problems. The treatment possibilities of MCs should be fitted to the problems of their visitors, and should not be limited to the pharmacological treatment for dementia.

In the last chapter of this thesis (Chapter 8), a general discussion of the main findings is provided. Methodological considerations with respect to study designs, selected subjects, 
used $\mathrm{MCl}$ criteria and statistical methods were made. We discussed the scientific and clinical relevance of the findings and made some recommendations for future research. 

Samenvatting 
Problemen in het cognitief functioneren komen vaak voor bij mensen van middelbare en oudere leeftijd. Het blijft een belangrijke vraag of deze cognitieve problemen vroege symptomen zijn van de ziekte van Alzheimer (AD) of niet. De overgangsfase tussen normaal cognitief functioneren en dementie wordt vaak Mild Cognitive Impairment (MCI) genoemd. In de laatste 20 jaar is er grote interesse ontstaan in mensen met $\mathrm{MCl}$, omdat deze mensen een verhoogd risico hebben om $A D$ te ontwikkelen. Echter niet alle mensen met $\mathrm{MCl}$ zullen in de toekomst $A D$ ontwikkelen. Om te kunnen differentiëren tusssen mensen die wel of geen $A D$ zullen ontwikkelen, is het belangrijk om voorspellers te identificeren voor toekomstige $A D$ in mensen met cognitive problemen. In het eerste deel van dit proefschrift wordt de voorspellende waarde van verschillende cognitieve en niet-cognitieve factoren voor AD onderzocht. In het tweede deel hebben we ons gericht op de ontwikkeling van de geheugenpolikliniek in Nederland en hebben we de kenmerken onderzocht van mensen die hulp zoeken bij een geheugenpolikliniek voor subjectieve geheugenklachten.

In hoofdstuk 2 is de voorspellende waarde van leerstrategieën voor AD onderzocht in mensen met $\mathrm{MCl}$ van de Maastrichtse geheugenpolikliniek. Mensen krijgen herhalingsonderzoeken gedurende 10 jaar. We vonden dat effortful strategieën, zoals subjectieve organisatie, die een actieve reorganisatie van de aangeboden informatie vereist, een significante voorspeller is van toekomstige $A D$ in mensen met $\mathrm{MCl}$. De meer passieve strategie, seriële clustering, was daarentegen geen voorspeller voor $A D$. Deze effecten zijn gelijk in mensen van middelbare en oudere leeftijd. Deze bevinding kan bijdragen bij het identificeren van mensen met prodromale $\mathrm{AD}$ in mensen met $\mathrm{MCl}$. Ook was er een associatie tussen het gebruik van subjectieve organisatie tijdens de leerfase en de uitgestelde geheugen prestatie. Deze informatie kan gebruikt worden bij de ontwikkeling van cognitieve training programma's.

In hoofdstuk 3 hebben we de relatie tussen het APOE e4 allel en geheugen disfunctioneren onderzocht in mensen met $\mathrm{MCl}$ van middelbare en oudere leeftijd van de Maastrichtse geheugenpolikliniek. In mensen van middelbare leeftijd was het APOE e4 allel het sterkst gerelateerd aan een verminderde subjectieve organisatie en in de oudere groep aan een verlaagde uitgestelde recall prestatie. Na de exclusie van mensen met onderliggende AD blijven de resultaten gelijk voor mensen van middlebare leeftijd, maar er was geen relatie meer tussen het APOE genotype en de geheugenmaten in de oudere subgroep. Wij hebben geconcludeerd dat het APOE e4 allel geassocieerd is met beperkingen in het geheugenfunctioneren, maar dat de geheugenmaat die betroffen is, varieert met de leeftijd. Wij concludeerden ook dat het effect van het APOE e4 allel op geheugenfunctioneren afhankelijk kan zijn van onderliggende $A D$ pathologie in oudere mensen, maar niet in mensen van middelbare leeftijd. Dit kan betekenen dat het APOE e4 allel een directe invloed heeft op het geheugenfunctioneren, onafhankelijk van $A D$ pathologie, of dat de follow-up periode voor mensen van middelbare 
leeftijd niet lang genoeg was om iedereen die klinische AD zal ontwikkelen, te identificeren. In dit geval zou subjectieve organisatie een hele vroege voorspeller van AD kunnen zijn.

In hoofdstuk 4 is de voorspellende waarde van affectieve symptomen voor AD onderzocht in mensen met $\mathrm{MCl}$ van de Maastrichtse geheugenpolikliniek. We hebben symptomen van depressie, angst, apathie, slaapproblemen en somatische problemen zoals gemeten met de HDRS onderzocht. De follow-up periode was 10 jaar. 228 mensen hadden tenminste een follow-up meting beschikbaar. 79 mensen ontwikkelden AD. Op baseline waren affectieve syptomen aanwezig in $50-70 \%$ van de mensen met $\mathrm{MCl}$. Slaapproblemen waren geassocieerd met een significant lager risico op AD. Depressieve en angst symptomen lieten een trend in dezelfde richting zien. De aanwezigheid van amnestische $\mathrm{MCl}$ had invloed op de relatie tussen depressieve symptomen en het risico op AD. Depressie was namelijk geassocieerd met een significant lager risico bij mensen zonder amnestische $\mathrm{MCl}$, maar niet in mensen met amnestische $\mathrm{MCl}$. Leeftijd en lengte van de follow-up periode had geen invloed op het risico van affectieve symptomen op AD. Wij concludeerden dat affectieve symptomen geen diagnosticum zijn voor prodromale $A D$, omdat deze symptomen geassocieerd waren met een verlaagd risico op AD. In tabel 3 van dit hoofdstuk wordt een overzicht gegeven van eerdere studies die de voorspellende waarde van depressieve symptomen op AD hebben onderzocht in mensen met $\mathrm{MCl}$. De gepoolde OR van deze studies laat zien dat depressie niet voorspellend is voor $A D$ in mensen met $\mathrm{MCl}$. Maar gezien de hoge prevalentie van deze symptomen in prodromale $A D$, sluit de aanwezigheid van deze symptomen de mogelijkheid van prodromale $A D$ zeker niet uit. Omdat affectieve symptomen vaak voorkomen in mensen met $\mathrm{MCl}$, zouden zorginstellingen voor mensen met cognitieve klachten aandacht moeten besteden aan deze symptomatologie en psychiatrische expertise beschikbaar moeten hebben om deze symptomen te diagnosticeren en behandelen.

In hoofdstuk 5 wordt de predictieve waarde van cognitieve beperkingen, affectieve symptomen, gedragsmatige problemen, vasculaire problemen, loopstoornissen en veranderingen in gewicht en eetlust voor dementie onderzocht in mensen van het RegistratieNet Huisartsenpraktijken (RNH). We hebben 74 mensen met dementie vergeleken met 125 controles, gematched voor leeftijd, geslacht en huisartsenpraktijk. In deze studie hebben we een periode van vijf jaar voorafgaande aan de diagnose dementie onderzocht. In de vijf jaar voorafgaande aan de diagnose dementie bezochten mensen met preklinische dementie hun huisarts vaker dan controle personen. Loopstoornissen waren de vroegste voorspeller voor dementie. Cognitieve klachten waren voorspellend vanaf drie jaar voor de diagnose, waarbij de voorspellende waarde het sterkste was in het eerste jaar voorafgaande aan de diagnose. In het eerste jaar voorafgaande aan de diagnose rapporteerden $58 \%$ van de mensen met preklinische dementie cognitieve klachten bij hun huisarts vergeleken met $2 \%$ van de controle personen. Alle an- 
dere symptomen, met uitzondering van vasculaire symptomen waren voorspellend in het jaar voorafgaande aan de diagnose. Wij hebben geconcludeerd dat preklinische dementie geassocieerd is met een toename van het aantal huisartsbezoeken vanaf tenminste vijf jaar voorafgaande aan de diagnose dementie. Loopstoornissen en cognitieve klachten zijn de vroegste symptomen van preklinische dementie in de huisartsenpraktijk. Gegeven het feit dat bijna de helft van de mensen met preklinische dementie geen cognitieve klachten rapporteert in het jaar voorafgaande aan de diagnose, vinden we dat de huisarts een actieve rol moet spelen bij het identificeren van mensen met een verhoogd risico op dementie.

In hoofdstuk 6 hebben we onderzocht welke factoren bepalen of mensen met subjectieve geheugenklachten (SMC) medische hulp zoeken. We hebben 33 mensen met SMC die hulp gezocht hebben bij een geheugenpolikliniek vergeleken met 85 controle personen met SMC van een populatiestudie die geen hulp hebben gezocht voor hun geheugenklachten. Zowel de mensen van de geheugenpolikliniek als ook de controlepersonen hadden geen objectieve stoornis op geheugentests en beide groepen hadden een vergelijkbare MMSE score. We onderzochten symptomen van depressie, angst, extraversie en neuroticisme, memory self-efficacy, kwaliteit van leven, veranderingen in geheugen en dagelijks functioneren beoordeeld door een naaste, life-events en een familie anamnese voor dementie. Mensen die hulp zochten voor hun SMC hadden een lagere memory self-efficacy en een lagere mentale kwaliteit van leven. Naasten van mensen die hulp zochten rapporteerden een grotere afname in dagelijks functioneren dan naasten van controle personen en mensen die hulp zochten waren vaker bezorgd omdat ze een positieve familie anamnese hadden voor dementie. Mate van depressie, angst, extraversie en neuroticisme waren vergelijkbaar in beide groepen. Wij concludeerden dat mensen die medische hulp zochten voor hun SMC gekenmerkt werden door een lagere memory self-efficacy en kwaliteit van leven, meer achteruitgang in dagelijks functioneren en bezorgdheid door een positieve familie anamnese voor dementie vergeleken met mensen die geen hulp zochten voor SMC. Deze bevindingen zijn belangrijk bij de ontwikkeling van adequate interventies voor mensen zonder objectiveerbare geheugenstoornis die een geheugenpolikliniek bezoeken voor geheugenklachten.

In hoofdstuk 7 is de ontwikkeling van geheugenpoliklinieken in Nederland onderzocht. Daarnaast hebben we onderzocht of en hoe deze voorzieningen een rol kunnen spelen in niet-demente mensen, zoals mensen met $\mathrm{MCl}$. We vonden dat sinds 1998 het aantal geheugenpoliklinieken is toegenomen van 12 naar 40 en dat het aantal verwijzingen per instelling ook is toegenomen. Tegenwoordig ligt de nadruk minder op academische centra. Dementie was de belangrijkste syndroom diagnose (62\%), maar ook lichtere cognitieve problemen, zoals $\mathrm{MCl}$ (23\%) en geen cognitieve stoornis (15\%) waren gebruikelijk. In vergelijking met 1998 is er tegenwoordig toenemende aandacht voor lichtere cognitieve problema- 
tiek. Er was een grote variabiliteit tussen de instellingen in de verdeling van de diagnoses dementie/MCl/geen cognitieve stoornis. Het concept $\mathrm{MCl}$ werd gebruikt in $92 \%$ van de instellingen. De meest voorkomende diagnostische classificatie van mensen met $\mathrm{MCl}$ was primair degeneratief, vasculair en niet gespecificeerd. De behandeling van mensen met $\mathrm{MCl}$ was in $84 \%$ van de instellingen anders dan de behandeling van mensen met dementie. De conclusie was dat geheugenpoliklinieken tegenwoordig deel uitmaken van het reguliere zorgsysteem voor patienten met dementie. Daarnaast spelen geheugenpoliklinieken ook een steeds grotere rol in de diagnostiek en therapeutische procedures van mensen met lichtere problematiek. De behandelmogelijkheden van geheugenpoliklinieken zouden afgestemd moeten zijn op de problematiek van de bezoekers en moet niet beperkt zijn tot de farmacologische behandeling van dementie.

In het laatste hoofdstuk van dit proefschrift (hoofdstuk 8) wordt een algemene discussie van de hoofdbevindingen gegeven. Methodologische overwegingen met betrekking tot designs van de studies, geselecteerde patiënten, gebruikte criteria voor $\mathrm{MCl}$ en statistische methoden worden beschreven. We discussieerden de wetenschappelijke en klinische relevantie van de bevindingen en beschreven aanbevelingen voor toekomstig onderzoek. 

References 
REFERENCES

Aalten, P., de Vugt, M. E., Jaspers, N., Jolles, J., \& Verhey, F. R. (2005). The course of neuropsychiatric symptoms in dementia. Part I: findings from the two-year longitudinal Maasbed study. Int J Geriatr Psychiatry, 20(6), 523-530.

Albert, M., Blacker, D., Moss, M. B., Tanzi, R., \& McArdle, J. J. (2007). Longitudinal change in cognitive performance among individuals with mild cognitive impairment. Neuropsychology, 21(2), 158169.

Albert, S. M., Glied, S., Andrews, H., Stern, Y., \& Mayeux, R. (2002). Primary care expenditures before the onset of Alzheimer's disease. Neurology, 59(4), 573-578.

Alexopoulos, G. S. (2006). The vascular depression hypothesis: 10 years later. Biol Psychiatry, 60(12), 13041305.

American Psychiatric Association (APA). (1987). Diagnostic and Statistical Manual of Mental Disorders IIIR (IIIrd revised edn). Washington DC: American Psychiatric Association.

American Psychiatric Association (APA). (1994). Diagnostic and Statistical Manual of Mental Disorders IV (IVth edn). Washington DC: American Psychiatric Association.

Apostolova, L. G., \& Cummings, J. L. (2007). Neuropsychiatric Manifestations in Mild Cognitive Impairment: A Systematic Review of the Literature. Dement Geriatr Cogn Disord, 25(2), 115-126.

Arrindell, W., \& Ettema, J. (2003). Symptoms Checklist (SCL-90). Handleiding bij een multidimensionele psychopathologie-indicator. Lisse, The Netherlands: Swets \& Zeitlinger.

Arrindell, W. A., \& Ettema, H. (1981). Dimensionele structuur, betrouwbaarheid en validiteit van de Nederlandse bewerking van de Symptom Checklist (SCL-90); gegevens gebaseerd op een fobische en een 'normale' populatie. Nederlands Tijdschrift voor de Psychologie, 36, 77-108.

Artero, S., \& Ritchie, K. (2003). The detection of mild cognitive impairment in the general practice setting. Aging Ment Health, 7(4), 251-258.

Barnes, L. L., Schneider, J. A., Boyle, P. A., Bienias, J. L., \& Bennett, D. A. (2006). Memory complaints are related to Alzheimer disease pathology in older persons. Neurology, 67(9), 1581-1585.

Barrett, J. J., Haley, W. E., \& Powers, R. E. (1996). Alzheimer's disease patients and their caregivers: medical care issues for the primary care physician. South Med J, 89(1), 1-9.

Bartzokis, G., Lu, P. H., Geschwind, D. H., Edwards, N., Mintz, J., \& Cummings, J. L. (2006). Apolipoprotein E genotype and age-related myelin breakdown in healthy individuals: implications for cognitive decline and dementia. Arch Gen Psychiatry, 63(1), 63-72.

Bekers, O., op den Buijsch, R. A., de Vries, J. E., Wijnen, P. A., \& van Dieijen-Visser, M. P. (2002). Capillary electrophoretic detection in apolipoprotein E genotyping. Electrophoresis, 23(12), 1878-1881.

Belleville, S. (2008). Cognitive training for persons with mild cognitive impairment. Int Psychogeriatr, 20(1), 57-66.

Bennett, D. A., Schneider, J. A., Wilson, R. S., Bienias, J. L., Berry-Kravis, E., \& Arnold, S. E. (2005). Amyloid mediates the association of apolipoprotein E e4 allele to cognitive function in older people. $J$ Neurol Neurosurg Psychiatry, 76(9), 1194-1199.

Bjorklund, D. F., \& Douglas, R. N. (1997). The Development of Memory Strategies. In The Development of Memory in Childhood (pp. 201-245). Hove: Psychological Press.

Blessed, G., Tomlinson, B. E., \& Roth, M. (1968). The association between quantitative measures of dementia and of senile change in the cerebral grey matter of elderly subjects. Br J Psychiatry, 114(512), 797-811. 
Bookheimer, S. Y., Strojwas, M. H., Cohen, M. S., Saunders, A. M., Pericak-Vance, M. A., Mazziotta, J. C., et al. (2000). Patterns of brain activation in people at risk for Alzheimer's disease. N Engl J Med, 343(7), 450-456.

Borenstein, A. R., Wu, Y., Mortimer, J. A., Schellenberg, G. D., McCormick, W. C., Bowen, J. D., et al. (2005). Developmental and vascular risk factors for Alzheimer's disease. Neurobiol Aging, 26(3), 325-334.

Bouma, A., Mulder, J., \& Lindeboom, J. (1996). Neuropsychologische Diagnostiek: Handboek. Amsterdam: Swets \& Zeitlinger.

Bousfield, A. K., \& Bousfield, W. A. (1966). Measurements of Clustering and of Sequential Constancies in Repeated Free Recall. Psychological Reports, 19, 935-942.

Brand, N., \& Jolles, J. (1985). Learning and retrieval rate of words presented auditorily and visually. J Gen Psychol, 112(2), 201-210.

Brandt, J., Spencer, M., \& Folstein, M. (1988). The Telephonic Interview for Cognitive Status. Neuropsychiatry, Neuropsychology and Behavioral Neurology, 1(2), 111-117.

Bruscoli, M., \& Lovestone, S. (2004). Is MCI really just early dementia? A systematic review of conversion studies. Int Psychogeriatr, 16(2), 129-140.

Buchman, A. S., Wilson, R. S., Bienias, J. L., Shah, R. C., Evans, D. A., \& Bennett, D. A. (2005). Change in body mass index and risk of incident Alzheimer disease. Neurology, 65(6), 892-897.

Buntinx, F., Kester, A., Bergers, J., \& Knottnerus, J. A. (1996). Is depression in elderly people followed by dementia? A retrospective cohort study based in general practice. Age Ageing, 25(3), 231-233.

Buntinx, F., Knottnerus, JA., Jolles, J., Van Boxtel, M., Muris, JWM., Schouten, HJA \& Hoppner, P. (1994). Dementia in General Practice: incidence, prevalence, social prevalence and co-morbidity. Arch Public Health, 52, 475-487.

Cargin, J. W., Collie, A., Masters, C., \& Maruff, P. (2007). The nature of cognitive complaints in healthy older adults with and without objective memory decline. J Clin Exp Neuropsychol, 1-13.

Carlesimo, G. A., Mauri, M., Graceffa, A. M., Fadda, L., Loasses, A., Lorusso, S., et al. (1998). Memory performances in young, elderly, and very old healthy individuals versus patients with Alzheimer's disease: evidence for discontinuity between normal and pathological aging. $J$ Clin Exp Neuropsychol, 20(1), 14-29.

Comijs, H. C., Deeg, D. J., Dik, M. G., Twisk, J. W., \& Jonker, C. (2002). Memory complaints; the association with psycho-affective and health problems and the role of personality characteristics. A 6-year follow-up study. J Affect Disord, 72(2), 157-165.

Commissaris, C. J., Verhey, F. R., Jr., Ponds, R. W., Jolles, J., \& Kok, G. J. (1994). Public education about normal forgetfulness and dementia: importance and effects. Patient Educ Couns, 24(2), 109-115.

Copeland, M. P., Daly, E., Hines, V., Mastromauro, C., Zaitchik, D., Gunther, J., et al. (2003). Psychiatric symptomatology and prodromal Alzheimer's disease. Alzheimer Dis Assoc Disord, 17(1), 1-8.

Cummings, J. L., Mega, M., Gray, K., Rosenberg-Thompson, S., Carusi, D. A., \& Gornbein, J. (1994). The Neuropsychiatric Inventory: comprehensive assessment of psychopathology in dementia. Neurology, 44(12), 2308-2314.

DeCarli, C., Frisoni, G. B., Clark, C. M., Harvey, D., Grundman, M., Petersen, R. C., et al. (2007). Qualitative estimates of medial temporal atrophy as a predictor of progression from mild cognitive impairment to dementia. Arch Neurol, 64(1), 108-115.

Deeks, J. J., Altman, D. G., \& Bradburn, M. J. (2001). Statistical methods for examing heterogenity and combining results from several studies in meta-anlysis. In M. Egger, G. D. Smith \& D. G. Altman (Eds.), Systematic reviews in health care meta-nalysis in context. London: BMJ. 
REFERENCES

Delis, D. C., Freeland, J., Kramer, J. H., \& Kaplan, E. (1988). Integrating clinical assessment with cognitive neuroscience: construct validation of the California Verbal Learning Test. J Consult Clin Psychol, 56(1), 123-130.

Delis, D. C., Kramer, J. K., Kaplan, E., \& Ober, B. A. (1987). California Verbal Learning Test. . New York: Psychological Corporation

Delis, D. C., Massman, P. J., Butters, N., Salmon, D. P., Cermak, L. S., \& Kramer, J. K. (1991). Profiles of Demened and Amnestic Patients of the California Verbal Learning Test: Implications for the Assessment of Memory Disorders. A Journal of Consulting and Clinical Psychology, 3(1), 19-26.

Derogatis, L. R., \& Cleary, P. A. (1977). Factorial invariance across gender for the primary symptom dimensions of the SCL-90. Br J Soc Clin Psychol, 16(4), 347-356.

Derouesne, C., Lacomblez, L., Thibault, S., \& LePoncin, M. (1999). Memory complaints in young and elderly subjects. Int J Geriatr Psychiatry, 14(4), 291-301.

Dickerson, B. C., Sperling, R. A., Hyman, B. T., Albert, M. S., \& Blacker, D. (2007). Clinical prediction of Alzheimer disease dementia across the spectrum of mild cognitive impairment. Arch Gen Psychiatry, 64(12), 1443-1450.

Dik, M. G., Jonker, C., Comijs, H. C., Bouter, L. M., Twisk, J. W., van Kamp, G. J., et al. (2001). Memory complaints and APOE-epsilon4 accelerate cognitive decline in cognitively normal elderly. Neurology, 57(12), 2217-2222.

Dixon, R. A., Hultsch, D. F., \& Hertzog, C. (1988). The Metamemory in Adulthood (MIA) Quesionnaire. Psychopharmacology Bulletin, 24, 671-688.

Draskovic, I., Vernooij-Dassen, M., Verhey, F., Scheltens, P., \& Rikkert, M. O. (2008). Development of quality indicators for memory clinics. Int J Geriatr Psychiatry, 23(2), 119-128.

Farlow, M. R., He, Y., Tekin, S., Xu, J., Lane, R., \& Charles, H. C. (2004). Impact of APOE in mild cognitive impairment. Neurology, 63(10), 1898-1901.

Feldman, H. H., Ferris, S., Winblad, B., Sfikas, N., Mancione, L., He, Y., et al. (2007). Effect of rivastigmine on delay to diagnosis of Alzheimer's disease from mild cognitive impairment: the InDDEx study. Lancet Neurol, 6(6), 501-512.

Feldman, H. H., \& Jacova, C. (2005). Mild cognitive impairment. Am J Geriatr Psychiatry, 13(8), 645-655.

Filbey, F. M., Slack, K. J., Sunderland, T. P., \& Cohen, R. M. (2006). Functional magnetic resonance imaging and magnetoencephalography differences associated with APOEepsilon4 in young healthy adults. Neuroreport, 17(15), 1585-1590.

Fleisher, A., Grundman, M., Jack, C. R., Jr., Petersen, R. C., Taylor, C., Kim, H. T., et al. (2005). Sex, apolipoprotein E epsilon 4 status, and hippocampal volume in mild cognitive impairment. Arch Neurol, 62(6), 953-957.

Fletcher, P. C., Shallice, T., \& Dolan, R. J. (1998). The functional roles of prefrontal cortex in episodic memory. I. Encoding. Brain, 121 ( Pt 7), 1239-1248.

Fletcher, P. C., Shallice, T., Frith, C. D., Frackowiak, R. S., \& Dolan, R. J. (1998). The functional roles of prefrontal cortex in episodic memory. II. Retrieval. Brain, 121 (Pt 7), 1249-1256.

Foley, D., Monjan, A., Masaki, K., Ross, W., Havlik, R., White, L., et al. (2001). Daytime sleepiness is associated with 3-year incident dementia and cognitive decline in older Japanese-American men. J Am Geriatr Soc, 49(12), 1628-1632.

Folstein, M. F., Folstein, S. E., \& McHugh, P. R. (1975). "Mini-mental state". A practical method for grading the cognitive state of patients for the clinician. J Psychiatr Res, 12(3), 189-198. 
Gabryelewicz, T., Styczynska, M., Luczywek, E., Barczak, A., Pfeffer, A., Androsiuk, W., et al. (2007). The rate of conversion of mild cognitive impairment to dementia: predictive role of depression. Int $J$ Geriatr Psychiatry, 22(6), 563-567.

Gallassi, R., Bisulli, A., Oppi, F., Poda, R., \& Di Felice, C. (2008). Subjective cognitive complaints, neuropsychological performance, affective and behavioural symptoms in non-demented patients. Int J Geriatr Psychiatry, 23(1), 95-101.

Ganguli, M. (2006). Mild cognitive impairment and the 7 uses of epidemiology. Alzheimer Dis Assoc Disord, 20(3 Suppl 2), S52-57.

Ganguli, M., Dodge, H. H., Shen, C., \& DeKosky, S. T. (2004). Mild cognitive impairment, amnestic type: an epidemiologic study. Neurology, 63(1), 115-121.

Ganguli, M., Du, Y., Rodriguez, E. G., Mulsant, B. H., McMichael, K. A., Vander Bilt, J., et al. (2006). Discrepancies in information provided to primary care physicians by patients with and without dementia: the Steel Valley Seniors Survey. Am J Geriatr Psychiatry, 14(5), 446-455.

Gao, S., Hendrie, H. C., Hall, K. S., \& Hui, S. (1998). The relationships between age, sex, and the incidence of dementia and Alzheimer disease: a meta-analysis. Arch Gen Psychiatry, 55(9), 809-815.

Geda, Y. E., Smith, G. E., Knopman, D. S., Boeve, B. F., Tangalos, E. G., Ivnik, R. J., et al. (2004). De novo genesis of neuropsychiatric symptoms in mild cognitive impairment (MCl). Int Psychogeriatr, 16(1), 51-60.

Geerlings, M. I., Schoevers, R. A., Beekman, A. T., Jonker, C., Deeg, D. J., Schmand, B., et al. (2000). Depression and risk of cognitive decline and Alzheimer's disease. Results of two prospective community-based studies in The Netherlands. Br J Psychiatry, 176, 568-575.

Geslani, D. M., Tierney, M. C., Herrmann, N., \& Szalai, J. P. (2005). Mild cognitive impairment: an operational definition and its conversion rate to Alzheimer's disease. Dement Geriatr Cogn Disord, 19(5-6), 383-389.

Glosser, G., Gallo, J. L., Clark, C. M., \& Grossman, M. (2002). Memory encoding and retrieval in frontotemporal dementia and Alzheimer's disease. Neuropsychology, 16(2), 190-196.

Graham, J. E., Rockwood, K., Beattie, B. L., Eastwood, R., Gauthier, S., Tuokko, H., et al. (1997). Prevalence and severity of cognitive impairment with and without dementia in an elderly population. Lancet, 349(9068), 1793-1796.

Green, R. C., Cupples, L. A., Kurz, A., Auerbach, S., Go, R., Sadovnick, D., et al. (2003). Depression as a risk factor for Alzheimer disease: the MIRAGE Study. Arch Neurol, 60(5), 753-759.

Health Counsil of the Netherlands (2002). Dementia. The Hague: Health Counsil of the Netherlands. Hamilton, M. (1960). A rating scale for depression. J Neurol Neurosurg Psychiatry, 23, 56-62.

Horikawa, E., Matsui, T., Arai, H., Seki, T., Iwasaki, K., \& Sasaki, H. (2005). Risk of falls in Alzheimer's disease: a prospective study. Intern Med, 44(7), 717-721.

Hosmer, D. W., \& Lemeshow, S. (1999). Applied Survivalanalysis. New York: Wiley.

Jelicic, M., Bosma, H., Ponds, R. W., Van Boxtel, M. P., Houx, P. J., \& Jolles, J. (2002). Subjective sleep problems in later life as predictors of cognitive decline. Report from the Maastricht Ageing Study (MAAS). Int J Geriatr Psychiatry, 17(1), 73-77.

Jolles, J., Houx, P. J., Boxtel, v. M. P. J., \& Ponds, R. W. (1995). Maastricht Aging Study: Determinants of Cognitive Aging. Maastricht, the Netherlands: Neuropsych Publishers.

Jonker, C., Geerlings, M. I., \& Schmand, B. (2000). Are memory complaints predictive for dementia? A review of clinical and population-based studies. Int J Geriatr Psychiatry, 15(11), 983-991. 
REFERENCES

Jorm, A. F., Butterworth, P., Anstey, K. J., Christensen, H., Easteal, S., Maller, J., et al. (2004). Memory complaints in a community sample aged 60-64 years: associations with cognitive functioning, psychiatric symptoms, medical conditions, APOE genotype, hippocampus and amygdala volumes, and white-matter hyperintensities. Psychol Med, 34(8), 1495-1506.

Jorm, A. F., Mather, K. A., Butterworth, P., Anstey, K. J., Christensen, H., \& Easteal, S. (2007). APOE genotype and cognitive functioning in a large age-stratified population sample. Neuropsychology, 21(1), $1-8$.

Jungwirth, S., Fischer, P., Weissgram, S., Kirchmeyr, W., Bauer, P., \& Tragl, K. H. (2004). Subjective memory complaints and objective memory impairment in the Vienna-Transdanube aging community. $J$ Am Geriatr Soc, 52(2), 263-268.

Kahn, R. L., Zarit, S. H., Hilbert, N. M., \& Niederehe, G. (1975). Memory complaint and impairment in the aged. The effect of depression and altered brain function. Arch Gen Psychiatry, 32(12), 1569-1573.

Korf, E. S., Wahlund, L. O., Visser, P. J., \& Scheltens, P. (2004). Medial temporal lobe atrophy on MRI predicts dementia in patients with mild cognitive impairment. Neurology, 63(1), 94-100.

Kramer, J. H., Delis, D. C., Kaplan, E., O'Donnell, L., \& Prifitera, A. (1997). Developmental sex differences in verbal learning. Neuropsychology, 11(4), 577-584.

Kramer, J. H., Rosen, H. J., Du, A. T., Schuff, N., Hollnagel, C., Weiner, M. W., et al. (2005). Dissociations in hippocampal and frontal contributions to episodic memory performance. Neuropsychology, 19(6), 799-805.

La Rue, A., Spar, J., \& Hill, C. D. (1986). Cognitive impairment in late-life depression: clinical correlates and treatment implications. J Affect Disord, 11(3), 179-184.

Lautenschlager, N. T., Flicker, L., Vasikaran, S., Leedman, P., \& Almeida, O. P. (2005). Subjective memory complaints with and without objective memory impairment: relationship with risk factors for dementia. Am J Geriatr Psychiatry, 13(8), 731-734.

Li, Y. S., Meyer, J. S., \& Thornby, J. (2001). Longitudinal follow-up of depressive symptoms among normal versus cognitively impaired elderly. Int J Geriatr Psychiatry, 16(7), 718-727.

Lindesay, J., Marudkar, M., van Diepen, E., \& Wilcock, G. (2002). The second Leicester survey of memory clinics in the British Isles. Int J Geriatr Psychiatry, 17(1), 41-47.

Liu, H. C., Wang, P. N., Wang, H. C., Lin, K. N., Hong, C. J., Liu, C. Y., et al. (2007). Conversion to dementia from questionable dementia in an ethnic Chinese population. J Geriatr Psychiatry Neurol, 20(2), 76-83.

Lopez, O. L., Becker, J. T., \& Sweet, R. A. (2005). Non-cognitive symptoms in mild cognitive impairment subjects. Neurocase, 11(1), 65-71.

Lyketsos, C. G., Lopez, O., Jones, B., Fitzpatrick, A. L., Breitner, J., \& DeKosky, S. (2002). Prevalence of neuropsychiatric symptoms in dementia and mild cognitive impairment: results from the cardiovascular health study. Jama, 288(12), 1475-1483.

Mariani, E., Monastero, R., \& Mecocci, P. (2007). Mild cognitive impairment: a systematic review. J Alzheimers Dis, 12(1), 23-35.

Martins, I. J., Hone, E., Foster, J. K., Sunram-Lea, S. I., Gnjec, A., Fuller, S. J., et al. (2006). Apolipoprotein E, cholesterol metabolism, diabetes, and the convergence of risk factors for Alzheimer's disease and cardiovascular disease. Mol Psychiatry, 11(8), 721-736. 
McKhann, G., Drachman, D., Folstein, M., Katzman, R., Price, D., \& Stadlan, E. M. (1984). Clinical diagnosis of Alzheimer's disease: report of the NINCDS-ADRDA Work Group under the auspices of Department of Health and Human Services Task Force on Alzheimer's Disease. Neurology, 34(7), 939-944.

Metsemakers, J. F., Hoppener, P., Knottnerus, J. A., Kocken, R. J., \& Limonard, C. B. (1992). Computerized health information in The Netherlands: a registration network of family practices. Br J Gen Pract, 42(356), 102-106.

Modrego, P. J., \& Ferrandez, J. (2004). Depression in patients with mild cognitive impairment increases the risk of developing dementia of Alzheimer type: a prospective cohort study. Arch Neurol, 61(8), 1290-1293.

Mol, M., Carpay, M., Ramakers, I., Rozendaal, N., Verhey, F., \& Jolles, J. (2007). The effect of perceived forgetfulness on quality of life in older adults; a qualitative review. Int J Geriatr Psychiatry, 22(5), 393-400.

Mondadori, C. R., Buchmann, A., Mustovic, H., Schmidt, C. F., Boesiger, P., Nitsch, R. M., et al. (2006). Enhanced brain activity may precede the diagnosis of Alzheimer's disease by 30 years. Brain, 129(Pt 11), 2908-2922.

Mulder, J. L., Dekker, R., \& Dekker, P. H. (1996). Verbale Leer en Geheugen Test. Lisse: Swets \& Zeitlinger.

Neary, D., Snowden, J. S., Gustafson, L., Passant, U., Stuss, D., Black, S., et al. (1998). Frontotemporal lobar degeneration: a consensus on clinical diagnostic criteria. Neurology, 51(6), 1546-1554.

Nederlands Huisartsen Genootschap, N. H. (1993). NHG-standaarden voor de huisarts. Utrecht: Bunge.

Nilsson, L. G., Adolfsson, R., Backman, L., Cruts, M., Nyberg, L., Small, B. J., et al. (2006). The influence of APOE status on episodic and semantic memory: data from a population-based study. Neuropsychology, 20(6), 645-657.

Ownby, R. L., Crocco, E., Acevedo, A., John, V., \& Loewenstein, D. (2006). Depression and risk for Alzheimer disease: systematic review, meta-analysis, and metaregression analysis. Arch Gen Psychiatry, 63(5), 530-538.

Palmer, K., Backman, L., Winblad, B., \& Fratiglioni, L. (2003). Detection of Alzheimer's disease and dementia in the preclinical phase: population based cohort study. Bmj, 326(7383), 245.

Palmer, K., Berger, A. K., Monastero, R., Winblad, B., Backman, L., \& Fratiglioni, L. (2007). Predictors of progression from mild cognitive impairment to Alzheimer disease. Neurology, 68(19), 1596-1602.

Panza, F., Capurso, C., D'Introno, A., Colacicco, A. M., Zenzola, A., Menga, R., et al. (2008). Impact of depressive symptoms on the rate of progression to dementia in patients affected by mild cognitive impairment. The Italian Longitudinal Study on Aging. Int J Geriatr Psychiatry, 23 (7), 726-734.

Passmore, A. P., \& Craig, D. A. (2004). The future of memory clinics. Psychiatric Bulletin, 28, 375-377.

Perri, R., Carlesimo, G. A., Serra, L., \& Caltagirone, C. (2005). Characterization of memory profile in subjects with amnestic mild cognitive impairment. J Clin Exp Neuropsychol, 27(8), 1033-1055.

Petersen, R. C. (2004). Mild cognitive impairment as a diagnostic entity. J Intern Med, 256(3), 183-194.

Petersen, R. C., Smith, G. E., Ivnik, R. J., Tangalos, E. G., Schaid, D. J., Thibodeau, S. N., et al. (1995). Apolipoprotein $E$ status as a predictor of the development of Alzheimer's disease in memoryimpaired individuals. Jama, 273(16), 1274-1278.

Petersen, R. C., Smith, G. E., Waring, S. C., Ivnik, R. J., Tangalos, E. G., \& Kokmen, E. (1999). Mild cognitive impairment: clinical characterization and outcome. Arch Neurol, 56(3), 303-308. 
REFERENCES

Petersen, R. C., Stevens, J. C., Ganguli, M., Tangalos, E. G., Cummings, J. L., \& DeKosky, S. T. (2001). Practice parameter: early detection of dementia: mild cognitive impairment (an evidence-based review). Report of the Quality Standards Subcommittee of the American Academy of Neurology. Neurology, 56(9), 1133-1142.

Ponds, R. W., Commissaris, K. J., \& Jolles, J. (1997). Prevalence and covariates of subjective forgetfulness in a normal population in The Netherlands. Int J Aging Hum Dev, 45(3), 207-221.

Ponds, R. W. H. M., \& Jolles, J. (1996a). Memory complaints in the elderly: the role of memory abolities, metamemory, depression, and personality. Educ Geront, 22, 341-357.

Ponds, R. W. H. M., \& Jolles, J. (1996b). The Abridged Dutch Metamemory in Adulthood (MIA) Questionnaire: structure and effects of age, sex, and education. Psychol Aging, 11(2), 324-332.

Ramakers, I., Visser, P., Aalten, P., Maes, H., Lansdaal, H., Meijs, C., et al. (submitted). Memory strategies in amnestic Mild Cognitive Impairment and their predictive value for Alzheimer's disease.

Reiman, E. M., \& Caselli, R. J. (1999). Alzheimer's disease. Maturitas, 31(3), 185-200.

Reiman, E. M., Chen, K., Alexander, G. E., Caselli, R. J., Bandy, D., Osborne, D., et al. (2004). Functional brain abnormalities in young adults at genetic risk for late-onset Alzheimer's dementia. Proc Natl Acad Sci U S A, 101(1), 284-289.

Reisberg, B., Ferris, S. H., de Leon, M. J., \& Crook, T. (1982). The Global Deterioration Scale for assessment of primary degenerative dementia. Am J Psychiatry, 139(9), 1136-1139.

Reitan, R. (1958). Validity of the Trial Making Test as an indication of organic brain damage. Percept Mot Skills, 8, 271 - 276.

Ribeiro, F., Guerreiro, M., \& De Mendonca, A. (2007). Verbal learning and memory deficits in Mild Cognitive Impairment. J Clin Exp Neuropsychol, 29(2), 187-197.

Ritchie, K., \& Fuhrer, R. (1992). A comparative study of the performance of screening tests for senile dementia using receiver operating characteristics analysis. J Clin Epidemiol, 45(6), 627-637.

Robert, P. H., Berr, C., Volteau, M., Bertogliati, C., Benoit, M., Sarazin, M., et al. (2006). Apathy in patients with mild cognitive impairment and the risk of developing dementia of Alzheimer's disease: a one-year follow-up study. Clin Neurol Neurosurg, 108(8), 733-736.

Roman, G. C., Tatemichi, T. K., Erkinjuntti, T., Cummings, J. L., Masdeu, J. C., Garcia, J. H., et al. (1993). Vascular dementia: diagnostic criteria for research studies. Report of the NINDS-AIREN International Workshop. Neurology, 43(2), 250-260.

Rosen, W. G., Mohs, R. C., \& Davis, K. L. (1984). A new rating scale for Alzheimer's disease. Am J Psychiatry, 141(11), 1356-1364.

Rozzini, L., Chilovi, B. V., Trabucchi, M., \& Padovani, A. (2005). Depression is unrelated to conversion to dementia in patients with mild cognitive impairment. Arch Neurol, 62(3), 505; author reply 505506.

Ruitenberg, A., Ott, A., van Swieten, J. C., Hofman, A., \& Breteler, M. M. (2001). Incidence of dementia: does gender make a difference? Neurobiol Aging, 22(4), 575-580.

Saan, R. J., \& Deelman, B. G. (1986). Nieuwe 15-woorden test A en B (15WT-A en 15WT-B) (N.-w. t. A. a. B. W.-A. a. 15WT-B), Trans.). In A. Bouma, J. Mulder \& J. Lindeboom (Eds.), Neuropsychologische Diagnostiek: Handboek (pp. 13-28). Amsterdam: Swets \& Zeitlinger.

Sarazin, M., Berr, C., De Rotrou, J., Fabrigoule, C., Pasquier, F., Legrain, S., et al. (2007). Amnestic syndrome of the medial temporal type identifies prodromal AD: a longitudinal study. Neurology, 69(19), 1859-1867. 
Sato, T. (2005). The Eysenck Personality Questionnaire Brief Version: factor structure and reliability. J Psychol, 139(6), 545-552.

Savage, C. R., Deckersbach, T., Heckers, S., Wagner, A. D., Schacter, D. L., Alpert, N. M., et al. (2001). Prefrontal regions supporting spontaneous and directed application of verbal learning strategies: evidence from PET. Brain, 124(Pt 1), 219-231.

Saxton, J., Lopez, O. L., Ratcliff, G., Dulberg, C., Fried, L. P., Carlson, M. C., et al. (2004). Preclinical Alzheimer disease: neuropsychological test performance 1.5 to 8 years prior to onset. Neurology, 63(12), 2341-2347.

Saykin, A. J., Wishart, H. A., Rabin, L. A., Santulli, R. B., Flashman, L. A., West, J. D., et al. (2006). Older adults with cognitive complaints show brain atrophy similar to that of amnestic $\mathrm{MCl}$. Neurology, 67(5), 834-842.

Scarmeas, N., Habeck, C. G., Hilton, J., Anderson, K. E., Flynn, J., Park, A., et al. (2005). APOE related alterations in cerebral activation even at college age. J Neurol Neurosurg Psychiatry, 76(10), 14401444.

Scarmeas, N., Habeck, C. G., Stern, Y., \& Anderson, K. E. (2003). APOE genotype and cerebral blood flow in healthy young individuals. Jama, 290(12), 1581-1582.

Scherder, E., Eggermont, L., Swaab, D., van Heuvelen, M., Kamsma, Y., de Greef, M., et al. (2007). Gait in ageing and associated dementias; its relationship with cognition. Neurosci Biobehav Rev.

Schmand, B., Jonker, C., Hooijer, C., \& Lindeboom, J. (1996). Subjective memory complaints may announce dementia. Neurology, 46(1), 121-125.

Schmand, B., Smit, J., Lindeboom, J., Smits, C., Hooijer, C., Jonker, C., et al. (1997). Low education is a genuine risk factor for accelerated memory decline and dementia. J Clin Epidemiol, 50(9), 10251033.

Schneider, W., \& Sodian, B. (1997). Memory Strategy Development: Lessons from Longitudinal Research. Developmental Review, 17, 442-461.

Shafer, A. B. (2006). Meta-analysis of the factor structures of four depression questionnaires: Beck, CES-D, Hamilton, and Zung. J Clin Psychol, 62(1), 123-146.

Slooter, A. J., Cruts, M., Kalmijn, S., Hofman, A., Breteler, M. M., Van Broeckhoven, C., et al. (1998). Risk estimates of dementia by apolipoprotein $\mathrm{E}$ genotypes from a population-based incidence study: the Rotterdam Study. Arch Neurol, 55(7), 964-968.

Small, B. J., Rosnick, C. B., Fratiglioni, L., \& Backman, L. (2004). Apolipoprotein E and cognitive performance: a meta-analysis. Psychol Aging, 19(4), 592-600.

Smith, G. E., Bohac, D. L., Waring, S. C., Kokmen, E., Tangalos, E. G., Ivnik, R. J., et al. (1998). Apolipoprotein E genotype influences cognitive 'phenotype' in patients with Alzheimer's disease but not in healthy control subjects. Neurology, 50(2), 355-362.

Steffens, D. C., Otey, E., Alexopoulos, G. S., Butters, M. A., Cuthbert, B., Ganguli, M., et al. (2006). Perspectives on depression, mild cognitive impairment, and cognitive decline. Arch Gen Psychiatry, 63(2), 130-138.

Sternberg, R. J., \& Tulving, E. (1977). The Measurement of Subjective Organization in Free Recall. Psychological Bulletin, 84(3), 539-556.

Storandt, M., Grant, E. A., Miller, J. P., \& Morris, J. C. (2006). Longitudinal course and neuropathologic outcomes in original vs revised $\mathrm{MCl}$ and in pre-MCl. Neurology, 67(3), 467-473.

Streams, M. E., Wackerbarth, S. B., \& Maxwell, A. (2003). Diagnosis-seeking at subspecialty memory clinics: trigger events. Int J Geriatr Psychiatry, 18(10), 915-924. 
REFERENCES

Stroop, J. (1935). Studies of interference in serial verbal reactions. J Exp Psychol, 18, 643-662.

Sun, X., Steffens, D. C., Au, R., Folstein, M., Summergrad, P., Yee, J., et al. (2008). Amyloid-associated depression: a prodromal depression of Alzheimer disease? Arch Gen Psychiatry, 65(5), 542-550.

Tabert, M. H., Albert, S. M., Borukhova-Milov, L., Camacho, Y., Pelton, G., Liu, X., et al. (2002). Functional deficits in patients with mild cognitive impairment: prediction of AD. Neurology, 58(5), 758-764.

Tabert, M. H., Manly, J. J., Liu, X., Pelton, G. H., Rosenblum, S., Jacobs, M., et al. (2006). Neuropsychological prediction of conversion to Alzheimer disease in patients with mild cognitive impairment. Arch Gen Psychiatry, 63(8), 916-924.

Teng, E., Lu, P. H., \& Cummings, J. L. (2007). Neuropsychiatric symptoms are associated with progression from mild cognitive impairment to Alzheimer's disease. Dement Geriatr Cogn Disord, 24(4), 253259.

Tierney, M. C., Boyle, E., Lam, R. E., \& Szalai, J. P. (1999). Do depressive symptoms in memory-impaired elders predict probable Alzheimer's disease? Aging \& Mental Health, 3(1), 88-93.

Tierney, M. C., Szalai, J. P., Snow, W. G., Fisher, R. H., Nores, A., Nadon, G., et al. (1996). Prediction of probable Alzheimer's disease in memory-impaired patients: A prospective longitudinal study. Neurology, 46(3), 661-665.

Tierney, M. C., Szalai, J. P., Snow, W. G., Fisher, R. H., Tsuda, T., Chi, H., et al. (1996). A prospective study of the clinical utility of ApoE genotype in the prediction of outcome in patients with memory impairment. Neurology, 46(1), 149-154.

Trivedi, M. A., Schmitz, T. W., Ries, M. L., Torgerson, B. M., Sager, M. A., Hermann, B. P., et al. (2006). Reduced hippocampal activation during episodic encoding in middle-aged individuals at genetic risk of Alzheimer's disease: a cross-sectional study. BMC Med, 4, 1.

Tulving, E. (1962). Subjective orhanization in free recall of "unrelated" words. . Psychological Review, 69, 344-354.

Twamley, E. W., Ropacki, S. A., \& Bondi, M. W. (2006). Neuropsychological and neuroimaging changes in preclinical Alzheimer's disease. J Int Neuropsychol Soc, 12(5), 707-735.

Tworoger, S. S., Lee, S., Schernhammer, E. S., \& Grodstein, F. (2006). The association of self-reported sleep duration, difficulty sleeping, and snoring with cognitive function in older women. Alzheimer Dis Assoc Disord, 20(1), 41-48.

United Nations Educational, Scientific and Cultural Organization (UNESCO). (1976). International Standard Classification of Education (ISCED) Paris: UNESCO.

Van de Zee, K. I., Sanderman, R., \& Heyink, J. (1993). Psychometrische kwaliteiten van de MOS 36item Short Form Health Survey (SF-36) in een Nederlandse populatie. Tijdschrift voor Sociale Gezondheidszorg, 71, 183-191.

Van den Akker, M., Metsemakers, J., Limonard, C. \& Knottnerus, J. (2004). General practice: a gold mine for research. Data and scientific use of the Registration Network Family Practices. Maastricht: Unigrafic.

Van der Elst, W., van Boxtel, M. P., van Breukelen, G. J., \& Jolles, J. (2005). Rey's verbal learning test: normative data for 1855 healthy participants aged 24-81 years and the influence of age, sex, education, and mode of presentation. J Int Neuropsychol Soc, 11(3), 290-302.

Van der Elst, W., Van Boxtel, M. P., Van Breukelen, G. J., \& Jolles, J. (2006a). Normative data for the Animal, Profession and Letter M Naming verbal fluency tests for Dutch speaking participants and the effects of age, education, and sex. J Int Neuropsychol Soc, 12(1), 80-89. 
Van der Elst, W., Van Boxtel, M. P., Van Breukelen, G. J., \& Jolles, J. (2006b). The Stroop color-word test: influence of age, sex, and education; and normative data for a large sample across the adult age range. Assessment, 13(1), 62-79.

van der Vlies, A. E., Pijnenburg, Y. A., Koene, T., Klein, M., Kok, A., Scheltens, P., et al. (2007). Cognitive impairment in Alzheimer's disease is modified by APOE genotype. Dement Geriatr Cogn Disord, 24(2), 98-103.

Verghese, J., Lipton, R. B., Hall, C. B., Kuslansky, G., Katz, M. J., \& Buschke, H. (2002). Abnormality of gait as a predictor of non-Alzheimer's dementia. N Engl J Med, 347(22), 1761-1768.

Verhey, F. R., Jolles, J., Ponds, R. W., Rozendaal, N., Plugge, L. A., de Vet, R. C., et al. (1993). Diagnosing dementia: a comparison between a monodisciplinary and a multidisciplinary approach. $J$ Neuropsychiatry Clin Neurosci, 5(1), 78-85.

Verhey, F. R. J., Nods, M., Ponds, R. W. H. M., \& Scheltens, P. (1999). Geheugenpoliklinieken in Nederland. Nederlands Tijdschrift voor Neurologie, 3, 169-174.

Verhey, F. R. J., Ramakers, I. H. G. B., Jolles, J., Scheltens, P., Vernooij-Dassen, M., \& Olde RIkkert, M. (2007). Geheugenpoli's in Nederland: ontwikkelingen sinds 1998. Tijdschrift voor Geriatrie en Gerontologie, 38, 237 - 245.

Verhey, F. R. J., Reyerser van Buuren, E., \& Jolles, J. (1987). De geheugenpolikliniek: multidisciplinaire benadering bij stoornissen van het geheugen en andere cognitieve stoornissen. Amsterdan: Gerontologisch Instituut.

Visser, P. J., Kester, A., Jolles, J., \& Verhey, F. (2006). Ten-year risk of dementia in subjects with mild cognitive impairment. Neurology, 67(7), 1201-1207.

Visser, P. J., Scheltens, P., \& Verhey, F. R. (2005). Do MCl criteria in drug trials accurately identify subjects with predementia Alzheimer's disease? J Neurol Neurosurg Psychiatry, 76(10), 1348-1354.

Visser, P. J., \& Verhey, F. (2007). Mild cognitive impairment as predictor for Alzheimer's disease in clinical practice: effect of age and diagnostic criteria. Psychological Medicine, 37, 1-10.

Visser, P. J., Verhey, F. R., Ponds, R. W., Cruts, M., Van Broeckhoven, C. L., \& Jolles, J. (2000). Course of objective memory impairment in non-demented subjects attending a memory clinic and predictors of outcome. Int J Geriatr Psychiatry, 15(4), 363-372.

Visser, P. J., Verhey, F. R., Ponds, R. W., Kester, A., \& Jolles, J. (2000). Distinction between preclinical Alzheimer's disease and depression. J Am Geriatr Soc, 48(5), 479-484.

Visser, P. J., \& Verhey, F. R. J. (2008). Mild cognitive impairment as predictor for Alzheimer's disease in clinical practice: effect of age and diagnostic criteria. Psychol Med, 38(1), 113-122.

Visser, P. J., Verhey, F. R. J., Jolles, J., \& Jonker, C. (2002). Course of minimal dementia and predictors of outcome. Int J Geriatr Psychiatry, 17, 835-841.

Waite, L. M., Grayson, D. A., Piguet, O., Creasey, H., Bennett, H. P., \& Broe, G. A. (2005). Gait slowing as a predictor of incident dementia: 6-year longitudinal data from the Sydney Older Persons Study. $J$ Neurol Sci, 229-230, 89-93.

Wang, P. N., Lirng, J. F., Lin, K. N., Chang, F. C., \& Liu, H. C. (2006). Prediction of Alzheimer's disease in mild cognitive impairment: a prospective study in Taiwan. Neurobiol Aging, 27(12), 1797-1806.

Welsh, K. A., Butters, N., Mohs, R. C., Beekly, D., Edland, S., Fillenbaum, G., et al. (1994). The Consortium to Establish a Registry for Alzheimer's Disease (CERAD). Part V. A normative study of the neuropsychological battery. Neurology, 44(4), 609-614.

Wenham, P. R., Price, W. H., \& Blandell, G. (1991). Apolipoprotein E genotyping by one-stage PCR. Lancet, 337(8750), 1158-1159. 
REFERENCES

Whitehouse, P. J., \& Moody, H. R. (2006). Mild Cognitive Impairment, a 'hardening of the categories'? Dementia, 5(1), 11-25.

Whitman, G. T., Tang, Y., Lin, A., \& Baloh, R. W. (2001). A prospective study of cerebral white matter abnormalities in older people with gait dysfunction. Neurology, 57(6), 990-994.

Wilkinson, D., Stave, C., Keohane, D., \& Vincenzino, O. (2004). The role of general practitioners in the diagnosis and treatment of Alzheimer's disease: a multinational survey. J Int Med Res, 32(2), 149159.

Wilson, R. S., Arnold, S. E., Beck, T. L., Bienias, J. L., \& Bennett, D. A. (2008). Change in depressive symptoms during the prodromal phase of Alzheimer disease. Arch Gen Psychiatry, 65(4), 439-445.

Wilson, R. S., Schneider, J. A., Barnes, L. L., Beckett, L. A., Aggarwal, N. T., Cochran, E. J., et al. (2002). The apolipoprotein E epsilon 4 allele and decline in different cognitive systems during a 6-year period. Arch Neurol, 59(7), 1154-1160.

Wishart, H. A., Saykin, A. J., McAllister, T. W., Rabin, L. A., McDonald, B. C., Flashman, L. A., et al. (2006). Regional brain atrophy in cognitively intact adults with a single APOE epsilon4 allele. Neurology, 67(7), 1221-1224.

Wishart, H. A., Saykin, A. J., Rabin, L. A., Santulli, R. B., Flashman, L. A., Guerin, S. J., et al. (2006). Increased brain activation during working memory in cognitively intact adults with the APOE epsilon4 allele. Am J Psychiatry, 163(9), 1603-1610.

Wright, N., \& Lindesay, J. (1995). A survey of memory clinics in the British Isles. Int J Geriatr Psychiatry, 10, 379-386.

Zimprich, D., Martin, M., \& Kliegel, M. (2003). Subjective cognitive complaints, memory performance, and depressive affect in old age: a change-oriented approach. Int J Aging Hum Dev, 57(4), 339-366. 


\section{Dankwoord}


Omdat een proefschrift niet alleen tot stand komt door de persoon op de kaft, wil ik hier een aantal mensen bedanken die ieder op een eigen manier hebben meegeholpen aan dit proefschrift.

Klinisch wetenschappelijk onderzoek kan niet gedaan worden zonder de patiënten die bereid zijn hieraan deel te nemen. Daarom wil hier allereerst alle patiënten bedanken die bereid zijn geweest deel te nemen aan de studies die beschreven zijn in dit proefschrift.

Frans Verhey, mijn eerste promotor. Frans, ik ben je zeer dankbaar voor de mogelijkheid die je mij hebt geboden om hier op deze manier klinisch wetenschappelijk onderzoek te doen. Een manier waarin ik veel vrijheid had, maar altijd op je steun en advies kon rekenen als ik daarom vroeg. De ideeën die je had en de vragen die je stelde, hebben me vaak aan het denken gezet en geleid tot nieuwe inzichten. Jelle Jolles, mijn tweede promotor. Jelle, ik ben je zeer dankbaar voor je interesse in het verloop van het AIO-traject en in het $\mathrm{MCl}$ onderzoek. Ook wil ik je bedanken voor het kritisch lezen en becommentariëren van mijn artikelen.

Pieter Jelle Visser, mijn co-promotor. Pieter Jelle, bedankt voor de samenwerking, de vele goede adviezen, je kritische blik en je interesse in de dagelijkse gang van zaken van het $\mathrm{MCl}$-onderzoek. Ook bedankt voor de snelle reacties als er vragen waren (waardoor de afstand Maastricht-Amsterdam minder groot leek). Ik heb je kritische en wetenschappelijke commentaar altijd erg gewaardeerd en zeker veel van je geleerd.

Verder wil ik de mensen bedanken die een grote steun zijn geweest in de dataverzameling van het project. Allereerst Heidi Lansdaal. Heidi, jij als onderzoeksassistente op dit project zorgde voor de planning, structuur en continuïteit van de herhalingsonderzoeken. Ik weet nog dat jij mij 4 jaar geleden de weg wees in de gang van zaken van het $\mathrm{MCl}$ followup project. Monique Onnink, ook jou wil ik bedanken voor de ondersteuning in de data verzameling gedurende de laatste fase van mijn AIO-schap. Laura, Iris, Saartje, Rosa, Joost, Sil, Noortje en Kirsten, jullie wil ik bedanken voor het vele werk dat jullie als stagiair(e) verricht hebben binnen het $\mathrm{MCl}$ onderzoek. Het enthousiasme waarmee jullie de vele patiënten getest of gebeld hebben, hebben gezorgd voor een goede continuïteit en kwaliteit van de data.

Nico, jij bent degene bij wie de deur letterlijk en figuurlijk altijd open staat. Bedankt voor je hulp bij de statistiek en voor het data management van de $\mathrm{MCI}$ projecten. Of het nu mijn SPSS syntaxen waren die niet functioneerden of de MAC zelf die niet deed wat ik bedoelde, jij wist altijd hoe het wel moest of een andere manier om tot dezelfde oplossing te komen. Ron, ook jij bedankt voor de ondersteuning in computerland en ook voor het telkens weer aanpassen van de Nederlandse geheugenpoli stippenkaart.

Verder wilikmijn collega's bedanken voor degezelligheid en de prettige samenwerking in de afgelopen 4 jaar. In het bijzonder wil ik hier Heidi en Rosa, mijn vaste kamergenoten noemen. Heidi, sinds jij van baan veranderd bent, weet ik, het project en de planten pas echt 
waar jij allemaal zorg voor droeg. Rosa, jij begon 3 jaar geleden als stagiaire op het $\mathrm{MCl}$ project en bent daarna op letterlijk dezelfde stoel verder gegaan als AIO. Jij en Heidi zorg(d)en altijd voor een prettige werksfeer, waarin goed gewerkt, maar ook gelachen kon worden. Ananda, geen directe collega binnen NP, maar wel collega AIO, lunch-maatje, en bovenal natuurlijk goede vriendin. Ananda en Rosa, bedankt dat jullie de 25ste paranimf willen zijn.

Verder wil ik Carla, Natalie, Bart, Dymphy, Pauline, Heidi J, Marleen, Claire, Jeroen, Sharon, Jennifer, Deliane en Geert bedanken voor zowel jullie wetenschappelijke bijdrage en discussie als ook voor de gezelligheid. En natuurlijk Lia, mijn eerste $\mathrm{MCl}$-maatje. Jouw bijdragen over statistiek, $\mathrm{MCl}$, of wetenschap in de, laten we zeggen meer algemene zin van het woord, waren naast helder en scherpzinnig ook vooral gezellig. Marleen, Ananda en Heidi (alweer), jullie ook bedankt voor de ontspanning tijdens het 'woon-werk verkeer'. Door ons carpoolen werd de dagelijkse file minder erg.

Ook wil ik de mensen van het secretariaat, de administratie en de poli bedanken voor hun inzet en medewerking. Els, Netty, Lindy, Anky, Laurent, Marco, Sje, Marion, Lenie en MarieJose, bedankt! En daarnaast wil ik ook de artsen en arts-assistenten van de geheugenpoli bedanken voor hun hulp en alertheid bij de inclusie van de $\mathrm{MCl}$-patiënten.

Tot slot wil ik nog twee mensen bedanken voor hun interesse en steun over de grenzen van dit proefschrift heen: pap en mam bedankt! 

Curriculum Vitae 
Inez Ramakers werd geboren op 19 november 1979 te Kerkrade. In 1998 behaalde zij haar gymnasium diploma aan het Gymnasium Rolduc in Kerkrade. Hierna begon zij haar studie Psychologie, afstudeerrichting Biologische Psychologie aan de Universiteit Maastricht. Onderzoeks- en klinische stage werden gevolgd aan het Universitätsklinikum der RWTH Aachen, afdeling medische psychologie. Hier bleef zij na het behalen van haar diploma in 2003 werkzaam als wetenschappelijk medewerkster. In 2004 werd zij aangesteld als Assistent in Opleiding (AIO) bij de vakgroep Psychiatrie \& Neuropsychologie van de Universiteit Maastricht. Het onderzoek dat zij hier heeft gedaan, staat beschreven in dit proefschrift. Tijdens deze AIO periode heeft zij ook deelgenomen aan de Master-opleiding 'Affective Neuroscience' van de universiteiten Maastricht en Florence. Hiervan zal eind 2008 het diploma worden behaald. Momenteel is haar aanstelling verlengd binnen het Alzheimer Centrum Limburg waar ze onder andere betrokken is bij de projecten Parelsnoer en CTMM. 


\section{Publications and abstracts}




\section{Publications}

Ramakers, IHGB., Visser, PJ., Aalten, P., Maes, HL., Lansdaal, HGM., Meijs, CJC., Jolles, J. \& Verhey, FRJ.The predictive value of memory strategies for Alzheimer's disease in subject with Mild Cognitive Impairment. (Submitted, Chapter 2)

Ramakers, IHGB., Visser, PJ., Aalten, P., Bekers, O., Sleegers, K., van Broeckhoven, KL., Jolles, J. \& Verhey, FRJ. The association between APOE genotype and memory dysfunction in subjects with Mild Cognitive Impairment is related to age and Alzheimer pathology. (Accepted in Dementia and other Geriatric Cognitive Disorders, Chapter 3).

Ramakers, IHGB., Visser, PJ., Aalten, P., Kester, A., Jolles, J. \& Verhey, FRJ. Affective symptoms as predictor of Alzheimer's disease in subjects with Mild Cognitive Impairment: a 10-year follow-up study. (Submitted, Chapter 4).

Ramakers, IHGB., Visser PJ., Aalten, P., Boesten, HMJ., Metsemakers, JFM., Jolles, J. \& Verhey, FRJ. (2007). Symptoms of preclinical dementia in general practice up to five years before dementia diagnosis. Dementia and other Geriatric Cognitive Disorders, 24, 300-306. (Chapter 5).

Ramakers, IHGB., Visser, PJ., Bittermann, AJN., Ponds, RWHM., van Boxtel, MPJ., Verhey, FRJ. Determinants of help-seeking behaviour in subjects with subjective memory complaints at a memory clinic: a case-control study. (Accepted in International Journal of Geriatric Psychiatry, Chapter 6).

Verhey, FRJ., Ramakers, IHGB., Jolles J., Scheltens, Ph., Vernooij-Dassen, M. \& Olde Rikkert, M. (2007). Geheugenpoli's in Nederland: ontwikkelingen sinds 1998. Tijdschrift voor Gerontology en Geriatrie, 38, 237 - 245. (Chapter 7).

Mol, M., Carpay, M., Ramakers, I., Rozendaal, N., Verhey, F. \& Jolles, J. (2007). The effect of perceived forgetfulness on quality of life in older adults; a qualitative review. International Journal of Geriatric Psychiatry, 22, 393-400.

Verhey, FRJ., Wolfs, CAG. \& Ramakers, IHGB. (2007). De geheugenpolikliniek. Modern Medicine. 5, 224-226.

Verhey, FRJ., Ramakers, IHGB., Jolles, J., Scheltens, PH., Vernooij-Dassen, M., Olde Rikkert, MGM. \& Blom, M. (2005). Geheugenpoliklinieken in Nederland: inventarisatie 2004. Neuropsych publishers, Maastricht.

\section{Abstracts}

Ramakers, IHGB., Visser, PJ., Aalten, P., Kester, A., Jolles, J. \& Verhey, FRJ. The predictive value of affective symptoms for Alzheimer's disease in subjects with Mild Cognitive Impairment: a 10-year followup study. The 11th International conference on Alzheimer's disease and related disorders (ICAD), Chicago, July 2008 
Ramakers, I., Visser, PJ., Aalten, P., Boesten, H., Metsemakers, J., Jolles, J. \& Verhey, F. Symptoms of preclinical dementia in general practice up to 5 years before diagnosis. The 2 nd International Congress on Gait and Mental Function, Amsterdam (NL), February 2008.

Ramakers, I. Memory clinics in the Netherlands: development in the past 10 years. Karel V Symposium, Utrecht (NL), September 2007

Ramakers, I., Visser, PJ., Drijgers, RL; Aalten, P., Jolles, J. \& Verhey, FRJ. Cognitive decline in carriers and non-carriers of the ApoE-e4 allel in subjects with MCl: a 10-year follow up study. 6th Dutch Endo-Neuro-Psycho (ENP) Meeting, Satellite Sympopsium Neuropsychology, Doorwerth (NL), June 2007.

Ramakers, I., Visser, PJ., Aalten, P., Maes, I., Jolles, J. \& Verhey, F. The predictive value of memory strategies for the development of dementia. The 10th International Conference on Alzheimer's disease and related disorders (ICAD), Madrid (Sp), July 2006.

Ramakers, I., Visser, PJ., Aalten, P., Maes, I., Jolles, J. \& Verhey, F. The predictive value of memory strategies for the development of dementia. 5th Dutch Endo-Neuro-Psycho (ENP) Meeting, Satellite Sympopsia Neuropsychology, Doorwerth (NL), June 2006.

Ramakers, I., Visser, PJ., Verhey, F. Non-cognitive symptoms in Mild Cognitive Impairment. International Psychogeriatric Association (IPA) Stockholm (S), September 2005.

Verhey, F., Ramakers, I. \& Dijkstra, J. Memory clinics in the Netherlands. International Psychogeriatric Association (IPA) Stockholm (S), September 2005.

Ramakers, I., Verhey, F., Metsemakers, Boesten, J. \& Aalten, P. Prodromes of dementia in general practice. Fourth Dutch Endo-Neuro-Psycho (ENP) Meeting, Satellite Sympopsia Neuropsychology, Doorwerth (NL), June 2005

Verhey, F. \& Ramakers, I. Update of a survey of memory clinics in the Netherlands. IPA Rotorua, New Sealand, April 2005. 